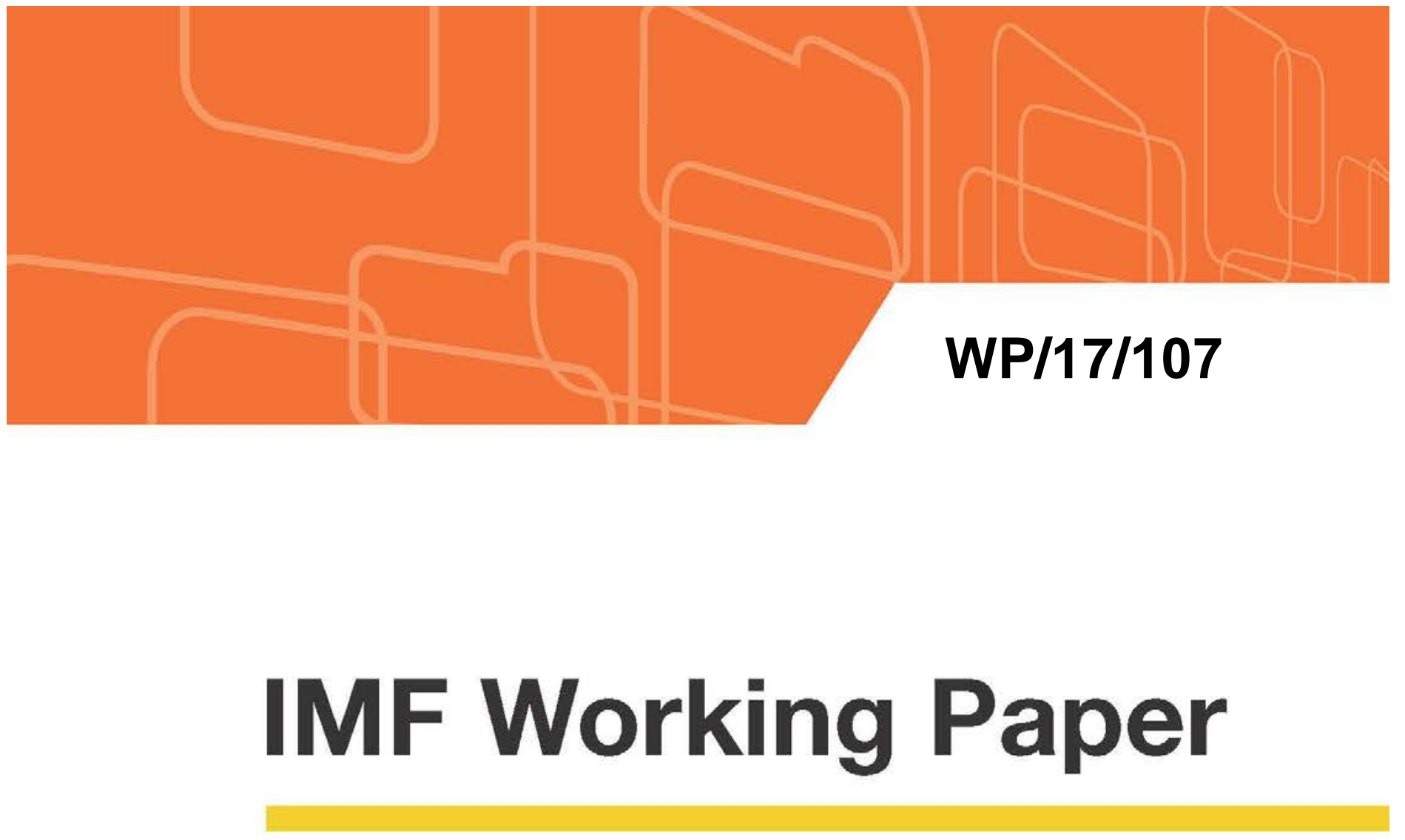

\title{
Variance Decomposition Networks: Potential Pitfalls and a Simple Solution
}

\author{
by Jorge A. Chan-Lau
}

IMF Working Papers describe research in progress by the author(s) and are published to elicit comments and to encourage debate. The views expressed in IMF Working Papers are those of the author(s) and do not necessarily represent the views of the IMF, its Executive Board, or IMF management. 


\title{
IMF Working Paper
}

\author{
Institute for Capacity and Development \\ Variance Decomposition Networks: Potential Pitfalls and a Simple Solution \\ Prepared by Jorge A. Chan-Lau \\ Authorized for distribution by Ralph Chami
}

May 2017

IMF Working Papers describe research in progress by the author(s) and are published to elicit comments and to encourage debate. The views expressed in IMF Working Papers are those of the author(s) and do not necessarily represent the views of the IMF, its Executive Board, or IMF management.

\begin{abstract}
Diebold and Yilmaz (2015) recently introduced variance decomposition networks as tools for quantifying and ranking the systemic risk of individual firms. The nature of these networks and their implied rankings depend on the choice decomposition method. The standard choice is the order invariant generalized forecast error variance decomposition of Pesaran and Shin (1998). The shares of the forecast error variation, however, do not add to unity, making difficult to compare risk ratings and risks contributions at two different points in time. As a solution, this paper suggests using the Lanne-Nyberg (2016) decomposition, which shares the order invariance property. To illustrate the differences between both decomposition methods, I analyzed the global financial system during 2001 - 2016. The analysis shows that different decomposition methods yield substantially different systemic risk and vulnerability rankings. This suggests caution is warranted when using rankings and risk contributions for guiding financial regulation and economic policy.
\end{abstract}

JEL Classification Numbers: C32, C39, G21

Keywords: networks, interconnectedness, systemic risk, regularization techniques, global financial system, variance decomposition, VAR

Author’s E-Mail Address: jchanlau@imf.org 


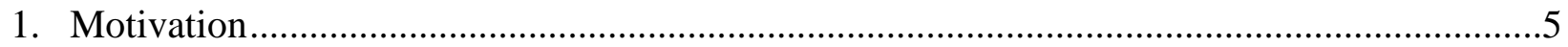

2. Network Interconnectedness and Systemic Risk: A Brief Literature Review .........................6

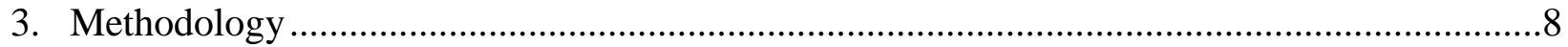

A High Dimensional VAR Estimation ................................................................

B.The Pesaran-Shin and Lanne-NybergGFEVDs.......................................................10

C. Variance Decomposition Networks .................................................................11

4. A Case Study: Systemic Risk in the Global Financial Network.........................................12

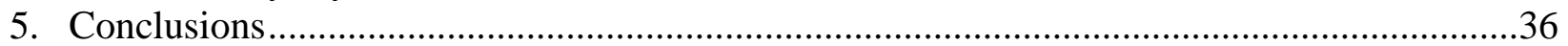

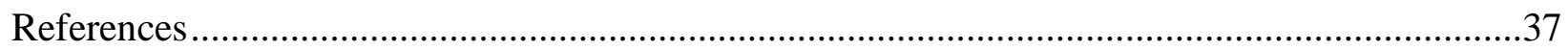

Appendix: Selected Tables, January 2001 - July 2016 .........................................................41

\section{Figures}

1. Distribution of Pesan-Shin total GFEVD contributions to the equity returns of individual firms, full sample and sub-sample periods

2. Number of overlapping firms in the top fifty DY and CLNDY rankings .............................19

3a. Banks: systemic risk rankings, probability distribution evolution......................................31

3b. Life insurers: systemic risk rankings, probability distribution evolution ...............................32

3c. Property/casualty/health insurers: systemic risk rankings, probability distribution

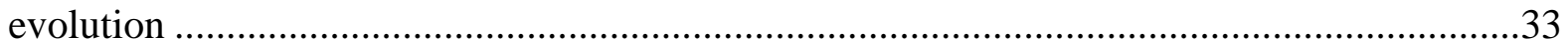

4a. Banks: systemic vulnerability rankings, probability distribution evolution ..........................33

4b. Life insurers: systemic vulnerability rankings, probability distribution evolution...................34

4c. Property/casualty/health insurers: systemic vulnerability rankings, probability distribution evolution

\section{Tables}

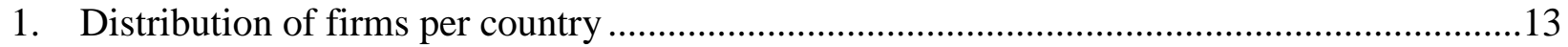

2. Rank correlations, Diebold-Yilmaz and corrected Lanne-Nyberg-Diebold-Yilmaz

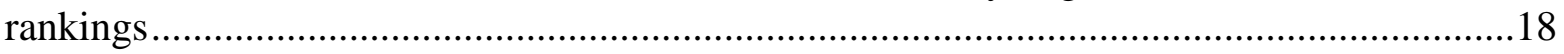

3a. Systemic risk rankings, all firms: January 2001 - December 2004 ....................................20

3b. Systemic risk rankings, all firms: January 2005 - December 2008....................................21

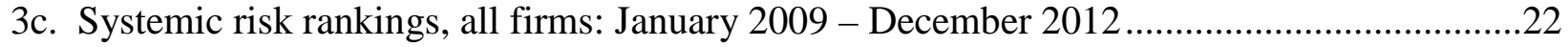

3d. Systemic risk rankings, all firms: January 2013 - July 2016 .........................................23

3e. Systemic risk rankings, all firms: January 2001 - July 2016 ...........................................24

4a. Systemic vulnerability rankings, all firms: January 2001 - December 2004 .......................25

4b. Systemic vulnerability rankings, all firms: January 2005 - December 2008 .......................26

4c. Systemic vulnerability rankings, all firms: January 2009 - December 2012 ......................27

4d. Systemic vulnerability rankings, all firms: January 2013 - July 2016 ...............................28

4e. Systemic vulnerability rankings, all firms: January 2001 - July 2016 ...............................29

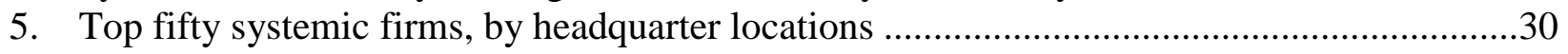

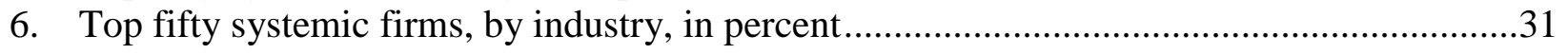

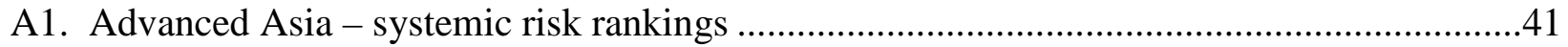

A2. Emerging markets economies - systemic risk rankings ...........................................42 


\section{Tables (continued)}

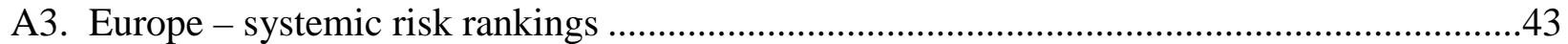

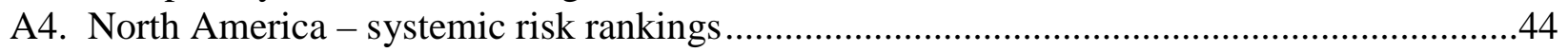

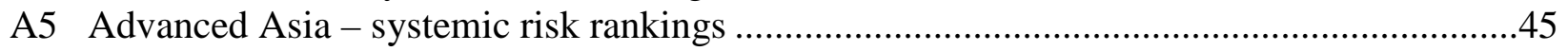

A6. Emerging markets economies - systemic risk rankings .......................................................46

A7. Europe - systemic risk rankings ………………..........................................................4

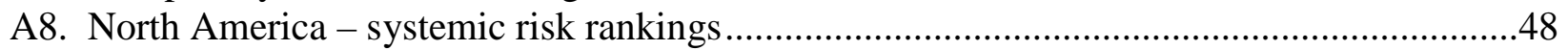




\section{Motivation ${ }^{1}$}

Following the global financial crisis in 2008, attention has focused on enhancing our understanding of systemic risk, and in particular, on how financial networks propagate and amplify adverse shocks. In the pursuit of these goals, the economics profession has built up on the rapid advances in network theory initially developed in biology and the computational social sciences, as illustrated in recent textbooks by Easley and Kleinberg (2010), and Jackson (2010).

Lessons and insights learned from the analysis of financial networks have already started to influence economic policy. The identification of systemic financial institutions, which are subject to higher capital requirements and enhanced oversight, incorporates interconnectedness as one of the assessment criteria (FSB, 2009). Central banks around the world are analyzing financial networks to assess contagion in the financial system, e.g. Banco de Mexico (2015), or the resilience of the banking sector to adverse shocks accounting for network spillovers, e.g. Anand, Bedard-Page, and Traclet (2014). From a multilateral perspective, Demekas et al (2013) apply clique percolation (Derenyi, Palla, and Vicsek, 2005) to identify the community of systemic financial jurisdictions using banking and portfolio exposures data.

Diebold and Yilmaz (2014) have recently proposed constructing variance decomposition networks that allow ranking firms by their systemic importance. For a group of firms, the construction of the network requires first estimating a vector autoregressive (VAR) model for their equity returns or other observable market-based measures. The impact of one firm on the network, or its systemic importance, is proportional to its contribution to the generalized forecast error variance decomposition (GFEVD) of the other firms. Two edges, hence, connect any two firms in the network. One edge measures the impact of the first firm on the GFEVD of the second firm, and the second edge measures the opposite.

One advantage of using the GFEVD as an interconnectedness measure is that the ordering of the variables in the VAR does not matter (Koop, Pesaran, and Potter, 1996). This is important when handling networks comprising a large number of firms for which it is unfeasible to specify a structural VAR model.

The order invariance comes at a cost though. Compared with the forecast error variance decomposition (FEVD) in a structural VAR, the GFEVD contributions do not add to unity since the error terms are correlated. This creates two potential pitfalls. The first one relates to the economic interpretation shocks to the system, a problem acknowledged in Koop et al (1996). The second one relates to how best to assess changes in the systemic contribution (or importance) of an individual firm over time.

To illustrate the last point, suppose that in one period firm A explains one fifth of the GFEVD of firm B while the sum of all the firms' contributions amounts to two. In another period, firm A explains one half of the GFEVD of firm B but contributions only add to one half. On the one hand, firm A seems more systemic in the second period since its contribution increased to fifty percent from twenty percent. On the other hand, firm A might be less systemic since it

\footnotetext{
${ }^{1}$ The paper benefits from discussions with Henri Nyberg on the statistical methodology, and the data collection work of Christina Daniels and Dulani Seneviratne. Any errors or omissions are the author's sole responsibility.
} 
contribution only accounts for one quarter (fifty percent of one half), down from forty percent (twenty percent of two).

In this paper, I point out that these pitfalls disappear when one uses the GFEVD of Lanne and Nyberg (2016) rather than the Koop-Pesaran-Potter GFEVD in an otherwise standard DieboldYilmaz (DY) variance decomposition network. In the modified network, i.e. the corrected Lanne-Nyberg (CLNDY) variance decomposition network, the GFEVD contributions sum to unity. Hence, shocks to individual equations have an economic interpretation similar to those in a structural VAR. It also becomes possible to compare the systemic importance of a firm, or systemic risk-based rankings, in two different periods of time in a consistent manner.

For comparison purposes, both DY and CLNDY networks were estimated for a large sample of publicly listed financial firms in advanced and emerging market economies during 2001 - 2016. The high dimensionality of the underlying VAR required using regularization techniques for estimating the coefficients and the variance-covariance matrix of the error terms. There is only weak correlation between systemic and vulnerability risk rankings implied from DY and CLNDY networks, indicating caution is warranted when risk ratings serve to guide policy.

The roadmap to the remainder of the paper is as follows. Section 2 reviews the literature on network interconnectedness and systemic risk in the financial system. Since research on this topic is accumulating at a rapid pace, the review would be, by necessity, brief and selective. Section 3 presents side by side the Koop-Pesaran-Potter and Lanne and Nyberg GFEVD methodologies, and argues why the latter might be more advantageous for constructing variance decomposition networks as first proposed by Diebold and Yilmaz (2014). This section also introduces the Lasso regularization and variance-covariance shrinking methods used to build the networks. Section IV puts the methodology to work by building both DY and CLNDY equityreturn networks and comparing their systemic risk rankings. Section V concludes.

\section{Network Interconnectedness and Systemic Risk: A Brief Literature Review}

Efforts towards understanding financial networks and its systemic implications have progressed rapidly along two different tracks. In the first track, networks emerge naturally from direct bilateral exposures between financial institutions and other market participants. Systemic risk in these networks arise from the potential realization of cascades of failures triggered by the inability of one firm to honor its obligations to other firms, as first advanced in Eisenberg and Noe (2001). Once a firm defaults subsequent failures arise from credit losses, forced sale of assets, funding withdrawals, and liquidity shortages (Brunnermeier and Pedersen, 2009).

Systemic risk rankings in these networks correspond to the total impact of a firms' failure on the system, measured as the losses their failure generate, weighted for instance by the centrality of the firms in the network as in Battiston et al (2012) for U.S. banks, and D’Errico, Battiston, and Gurciullo (2016) for European banks. In recent work, Jo (2012) captures the impact of counterparty risk, funding costs, and liquidity shortages in a direct exposures network, which, as in related models, must be solved numerically (Upper, 2011).

Networks based on direct exposures can capture the strategic and adaptive behavior of firms in the system, as done by Gai and Kapadia (2010) and Jo (2012) using numerical simulations; or 
Elliott et al (2014), and Acemoglu et al (2015) at a theoretical level. More recently, these networks arise endogenously in agent-based models incorporating complexity and real world constraints, such as margin calls or regulatory requirements, as in Montagna and Kok, (2013), Bookstaber and Maddrik (2015), and Chan-Lau (2015) among others.

Constructing direct exposures networks requires detailed counterparty exposure data. In many instances, the data would be available only for the aggregate counterparty exposure of an individual firm. There are methods available to reconstruct the bilateral exposures from aggregate data, but the reconstructed data is likely to differ substantially from the actual exposures (Anand, Craig, and Peter, 2015). Even if data on bilateral exposures are available, absent information on the characteristics of the bilateral exposures makes difficult to assess the extent of losses in the case of counterparty default. Lastly, the network may not be able to capture completely the transmission of shocks arising from common exposures to risk factors rather than bilateral exposures.

The second track of analytical work on financial networks bypasses these problems by relying on the comovement of firms' security prices. With efficient markets, prices would capture the risks from bilateral exposures as well as from indirect exposures, such as similarity in business models and common exposures to risk factors owing to similar trading, investment, and risk management strategies. In the network, the links between firms are proportional to the comovement of their security prices. While easier to build than direct exposures network, comovement networks cannot accommodate the strategic behavior of firms.

Mantegna (1999) pioneered the work on comovement networks, setting the strength of the link between two firms, or distance, proportional to the inverse of their stock price correlation. More recently, Billio et al (2012) built Granger-causality networks based on monthly asset returns for publicly listed financial firms and hedge funds. In these networks, the strength and directionality of the link between two firms is determined by the degree of their pair-wise Granger causality measures. The pair-wise estimation of these measures allows constructing networks with a large number of constituent firms. Nevertheless, pairwise effects are local in nature, ignoring the effects of other firms and neglecting the global aspect of the network. Indeed, pairwise Granger causality measure may be significant owing to the unobserved effect of a third firm.

Partial correlation analysis, a statistical technique introduced by Baba, Shibata, and Sibuya (2004), bypass the limitations of pairwise Granger-causality networks and earlier work on correlation networks. In simple words, the partial correlation is the residual correlation between two firms after subtracting the correlation with a third firm. The application of partial correlation analysis, therefore, isolates the link between two firms. What is lost, compared to Granger-causality networks, is the directionality of the link. Examples include Kennet et al (2010), who introduced partial correlation analysis in financial networks of stock returns, and those surveyed in Kennet et al (2014). Partial correlation analysis, however, could yield a network with all firms directly connected to each other, or in other words, the financial network reduces to a single giant component.

To trim the partial correlation network, Chan-Lau, Chuang, Duan, and Sun (2016) propose using a Lasso penalty restriction based on the CONCORD algorithm (Khare et al, 2015; Oh et al, 
2014) such that the partial correlation of weakly connected firms, i.e. low partial correlation values, is set equal to zero. If the penalty is too large, the network may generate a substantial number of orphan firms, i.e. firms completely disconnected from the network, which is at odds with the prior that the financial system is fully integrated. To avoid this situation, the value of the Lasso penalty is set to the maximum value such that all firms belong to the network. This restriction is consistent with the prior that the financial system is fully integrated albeit not fully connected. The authors apply these techniques to build networks based on the partial correlation of projected probabilities of default.

Diebold and Yilmaz (2014) propose to summarize the interconnectedness between firms using the variance decomposition in a VAR model of the financial system. Specifically, the contribution of one firm to the GFEVD of another firm is a directed measure of their interconnectedness to rule out the effect of the order of variables in the VAR (Koop, Pesaran, and Potter, 1996). Compared with plain correlation networks, there are two separate weighted edges connecting two firms in a variance decomposition network. One edge measures the effect of the first firm on the second, and the other measuring the reverse effect. As in the case of Granger causality networks, the notion of firms as sources and recipients of risk arise naturally in the Diebold-Yilmaz framework. The authors analyze the interconnectedness between ten U.S. financial firms using a VAR model for realized equity return volatility. Demirer, Diebold, Liu, and Yilmaz (2015), by leveraging on dimensionality reduction methods, are able to extend Diebold-Yilmaz original framework to a global bank network comprising the top one hundred banks worldwide.

Despite the positive features of Diebold-Yilmaz financial networks, weaknesses in the economic interpretation of the shocks remain, which have implications for systemic risk rankings based on the GFEVD, as pointed in the introductory section. I explain next why a simple modification based on the variance decomposition method proposed by Lanne and Nyberg (2016) goes a long way towards enhancing the original Diebold-Yilmaz analytical framework.

\section{Methodology}

The construction of variance decomposition networks requires three steps. First, it is necessary to estimate the VAR model, which requires the use of regularization techniques when the number of endogenous variables is large. Second, the estimates of the VAR coefficients serve to generate the GFEVD. Two options are available for performing the decomposition, one proposed by Pesaran and Shin (1998) and another proposed by Lanne and Nyberg (2016). In the third step, the GFEVD determines the network structure and the systemic importance of the firms. This section reviews the three steps.

\section{A. High-Dimensional VAR estimation}

Realizing the power of networks to uncover interconnectedness in the financial system requires including a large number of firms in the analysis. In variance decomposition networks, it demands estimating a high dimensional VAR model. Such estimation is challenging, with the 
number of autoregressive coefficients for each equation increasing quadratically in the number of lags, i.e. for $n$ variables and $p$ lags, the total number of coefficients equal to $n+n \times p^{2}$.

Bayesian VAR methods, surveyed in Koop (2013), are useful for estimating large VARs, and have proven better at forecasting than factor models when combining the use of Minnesota priors and stochastic search variable selection. But these methods could be computationally expensive relative to shrinkage methods incorporating the least absolute shrinkage and selection operator (lasso) of Tibshirani (1996), a widely used penalized least squares estimator for dealing with sparse high dimensional systems in economics (Fan, Ly, and Qi, 2011). Work exploring the application of lasso to VARs include Song and Bickel (2011), Li and Chen (2014), and Davis, Zang, and Zheng (2015). Nicholson, Matteson and Bien (2016) introduce the VARX-L to account for exogenous variables.

This study uses the Lasso to estimate each single equation in the VAR. The minimization of the penalized residual sum of squares in an otherwise least squares regression, i.e.

$$
\min _{\beta_{i, 0}, \beta_{i, k j}} \sum_{t=1}^{T}\left(y_{i, t}-\beta_{i, 0}-\sum_{k=1}^{p} \sum_{j=1}^{n} \beta_{i, k j} y_{j, t-k}\right)^{2}+\lambda \sum_{k=1}^{p} \sum_{j=1}^{n}\left|\beta_{i, k j}\right|
$$

yields the lasso coefficients $\beta_{i, k j}^{\lambda}$ in the $i$-th equation of the VAR model, where $T$ is the number of observations, and $p$ the number of lags. Note that the coefficients depend on the value of the penalty parameter, $\lambda$. The larger the parameter value, the larger the number of coefficients shrinked towards the value of zero.

Typically, $k$-fold cross-validation serves to choose the penalty parameter. The validation process divides the set of observation into $k$ groups or folds. Each fold serves successively as a validation set, with the model estimated using the remaining $k-1$ folds. The average cross-validation error is the average of the mean square error over the $k$ estimations. There are two commonly used criteria for parameter selection. The first one is to choose the parameter that yields the minimum cross-validation error. The second one, the one standard error rule, is to choose the minimum parameter with an associated error not exceeding the minimum cross-validation error by more than one standard deviation (see James et al, 2013, for a textbook exposition).

When estimating VAR models for weekly equity returns, the minimum the minimum crossvalidation error and one standard error criteria performed aggressively. In some instances, they shrank all autoregressive coefficients to zero, implying equity returns followed a random walk. The estimation also used the elastic net (Zou and Hastie, 2005) and the adaptive elastic net estimators (Zou and Zhang, 2009) as suggested by Demirer et al (2015), who showed it performed well when analyzing VAR models of daily equity return volatility. Both estimators, however, exhibited a behavior similar to the Lasso models under the minimum cross validation error and the one standard error rule, aggressively shrinking the coefficients to zero. 
Therefore, I adopted a simpler, somewhat ad hoc rule that set the penalty parameter equal to the maximum value such that the number of non-zero coefficients exceeds an arbitrary minimum threshold. The numerical results reported below correspond to a threshold value of twenty. After selecting the penalty parameter and the number of lagged values in the VAR, it is straightforward to estimate the individual equations.

\section{B. The Pesaran-Shin and Lanne-Nyberg GFEVDs}

Upon estimation of the VAR, it is possible to calculate the GFEVD of the system using either the Pesaran and Shin (1998) or Lanne and Nyberg (2016) decompositions. Both build on the generalized impulse response function (GIRF) proposed by Koop, Pesaran and Potter (1996).

To obtain the Pesaran-Shin GFEVD, start with the moving average representation of the VAR (Hamilton, 1994):

$$
\boldsymbol{Y}_{t}=\sum_{j=0}^{\infty} A_{j} \varepsilon_{t-j}
$$

where $\boldsymbol{Y}_{t}$ is the $n \times 1$ vector of the realization of the endogenous variables at time $t, \boldsymbol{A}_{\boldsymbol{j}}$ are $n \times n$ matrices, and $\boldsymbol{\varepsilon}_{\boldsymbol{t}}$ is an independent and identically distributed error term with zero mean and covariance matrix $\boldsymbol{\Sigma}=\left\{\sigma_{i j}, i, j=1, \ldots, n\right\}$. Koop et al (1996) specify the order-invariant generalized impulse response function $h$ periods ahead, GI, to a shock $\boldsymbol{\delta}$ at time $t$ given the past history of the economy at time $t-1, \boldsymbol{\Omega}_{t-1}$, as:

$$
G I\left(h, \boldsymbol{\delta}_{t}, \boldsymbol{\Omega}_{t-1}\right)=E\left(\boldsymbol{Y}_{t+h} \mid \boldsymbol{\varepsilon}_{t}=\boldsymbol{\delta}_{t}, \boldsymbol{\Omega}_{t-1}\right)-E\left(\boldsymbol{Y}_{t+h} \mid \boldsymbol{\Omega}_{t-1}\right)
$$

In a linear model, the GIRF is independent of the past history of shocks. Pesaran and Shin (1998) simplify the GIRF by restricting the shock to a single element, i.e. the $j$-th element, $\delta_{j}$ :

$$
G I\left(h, \boldsymbol{\delta}_{t}, \boldsymbol{\Omega}_{t-1}\right)=\boldsymbol{A}_{h} \boldsymbol{\Sigma} \boldsymbol{e}_{j} \sigma_{j j}^{-1} \delta_{j}
$$

where $\boldsymbol{e}_{\boldsymbol{j}}$ is a $n \times 1$ vector with all entries set to zero except for the $\boldsymbol{j}$-th entry. The corresponding Pesaran-Shin GFEVD from variable $j$ to variable $i$ at horizon $h, \theta_{i j}(h)$, follows after setting the shock $\delta_{j}$ equal to $\sqrt{\sigma_{j j}}$ :

$$
\theta_{i j}(h)=\frac{\sigma_{i i}^{-1} \sum_{k=0}^{h}\left(\boldsymbol{e}_{\boldsymbol{j}}^{\prime} \boldsymbol{A}_{k} \boldsymbol{\Sigma} \boldsymbol{e}_{\boldsymbol{j}}\right)^{2}}{\sum_{k=0}^{h} \boldsymbol{e}_{\boldsymbol{j}}^{\prime} \boldsymbol{A}_{k} \boldsymbol{\Sigma} \boldsymbol{A}_{k}^{\prime} \boldsymbol{e}_{\boldsymbol{i}}}, \quad i, j=1, \ldots, n .
$$

Compared with impulse responses obtained from a Cholesky decomposition, the GIRFs are unique and independent from the ordering of the variables in the VAR, and exactly equivalent if shocks are orthogonal. The invariance property extends to the Pesaran-Shin GFEVDs, but there is a cost to pay: they do not add to unity, i.e. $\sum_{j=1}^{n} \theta_{i j}(h) \neq 1$, unless the shocks are orthogonal 
to start with. Because of this feature, the interpretation of the Pesaran-Shin GFEVD as the partial contribution of a variable is at best ambiguous. To deal with this issue, Diebold and Yilmaz (2015) propose a standardized GFEVD given by:

$$
\tilde{\theta}_{i j}(h)=\frac{\theta_{i j}(h)}{\sum_{k=1}^{n} \theta_{i k}(h)}, \quad i, j=1, \ldots, n .
$$

Lanne and Nyberg (2016) introduce an alternative GFEVD, $\lambda_{i j}(h)$, based on the partial contribution of variable $j$ to the total GIRF of variable $i$ :

$$
\lambda_{i j}(h)=\frac{\sum_{k=0}^{h} G I\left(h, \boldsymbol{\delta}_{j t}, \boldsymbol{\Omega}_{t-1}\right)}{\sum_{j=1}^{n} \sum_{k=0}^{h} G I\left(h, \boldsymbol{\delta}_{j t}, \boldsymbol{\Omega}_{t-1}\right)}, \quad i, j=1, \ldots, n .
$$

The Lanne-Nyberg GFEVD adds to unity, and its interpretation as the relative contribution of a variable is straightforward and analogous to that of the Cholesky decomposition.

Calculating the GFEVDs requires estimates of the variance-covariance matrix $\boldsymbol{\Sigma}$ to obtain the GIRFs. In large dimensional systems, a simple empirical estimate may fail to be positive definite. To avoid this problem, the estimation in this analysis uses the shrinkage methods proposed by Schafer and Strimmer (2005) and Opgen-Rhein and Strimmer (2007).

\section{Variance Decomposition Networks}

Following Diebold and Yilmaz (2015), the pairwise directional connectedness from firm $j$ to firm $i$, is equal to its contribution to the $h$-step ahead GFEVD of equity returns:

$$
C_{i j}(h)=\left\{\begin{array}{l}
\tilde{\theta}_{i j}(h) \text { if using Pesaran-Shin GFEVD } \\
\lambda_{i j}(h) \text { if using Lanne-Nyberg GFEVD }
\end{array}, \quad i, j=1, \ldots, n .\right.
$$

where the decompositions correspond to equations (5) and (6) respectively. The Pesaran-Shin GFEVD yields the Diebold-Yilmaz (DY) network, and the Lanne and Nyberg GFEVD yields the corrected Lanne-Nyberg-Diebold-Yilmaz (CLNDY) network.

The total directional connectedness from a firm $\mathrm{j}$, is defined as the sum of its contribution to the $h$-step ahead GFEVD of the other firms' equity returns:

$$
C_{j}(h)=\frac{\sum_{i \neq j, i=1}^{n} C_{i j}(h)}{\sum_{i, j=1}^{n} C_{i j}(h)} \times 100, \quad i, j=1, \ldots, n .
$$

This measure captures the systemic risk of the firm, i.e. its total contribution to the forecast error variance of other firms in the system. Higher values correspond to higher systemic risk, making possible to rank firms by their systemic risk. Similarly, the systemic vulnerability of firm $i$ is 
simply the total contribution of other firms in the system to its forecast error variance, and is given by:

$$
V_{i}(h)=\frac{\sum_{i \neq j, j=1}^{n} C_{i j}(h)}{\sum_{i, j=1}^{n} C_{i j}(h)} \times 100, \quad i, j=1, \ldots, n .
$$

This measure helps us build a systemic vulnerability ranking, with more vulnerable firms characterized by higher values of $V_{i}(h)$. As in any statistical model, these risk and vulnerability measures assume that statistical causality implies economic causality. Even if this were not the case, however, the systemic risk and vulnerability measures would capture the role firms play in the amplification of shocks.

\section{A Case Study: Systemic Risk in the Global Financial Network}

To evaluate the similarities and differences between the systemic risk and vulnerability rankings implied by the DY and CLNDY networks, I estimated them using weekly equity price returns in U.S. dollars of publicly traded financial firms. The data sample comprised 402 firms headquartered in 34 advanced and emerging market economies and covered the period January 1 , 2001 - July 31, 2016. The sample of firms included those publicly traded as of mid-July 2015, which could introduce survivorship bias since it omit firms that merged or went into liquidation during the 2008 financial crisis, such as Lehman Brothers, Merrill Lynch and Dexia. The firms fall in one the following categories: (1) property/casualty/health insurers; (2) life insurers; (3) banks, and savings and loan associations; and (4) security brokers and dealers.

The analysis relied on systemic rankings estimated on the full sample as well as on four distinct periods. The periods were: (1) the pre-crisis period, i.e. January 1, 2001 to December 31, 2004; (2) the Lehman Brothers period, i.e. January 1, 2005 to December 31, 2008; (3) the European sovereign debt crisis period, i.e. January 1, 2009 to December 31, 2012; and (4) the secular stagnation period, January 1, 2013 to July 31, 2016. In each sub-period, the number of firms was 275, 331, 380, and 402 respectively. To avoid thinly traded firms, the analysis included only firms for which at least 80 percent of the observations in the subsample were different from zero. The horizon for the variance decompositions was one year (52 weeks).

Table 1 presents detailed information on the sample composition by country in each sub-sample period. The United States and emerging market economies headquartered one quarter of the firms each. Banks and property/casualty/health insurance firms each account for roughly forty percent of the firms in the sample. Life insurance companies account for most of the remaining twenty percent, with a few securities brokers and dealers making up for the rest. 
Table 1. Distribution of firms per country

Panel A: Sample period: Jan. 1, 2001 - Dec. 31 - 2004

\begin{tabular}{|c|c|c|c|c|c|}
\hline \multirow[b]{2}{*}{ Country } & \multicolumn{5}{|c|}{ Type of firm } \\
\hline & $\begin{array}{c}\text { Insurance: } \\
\text { Property and } \\
\text { Casualty; Health }\end{array}$ & $\begin{array}{c}\text { Life } \\
\text { Insurance }\end{array}$ & $\begin{array}{c}\text { Banks and } \\
\text { Savings\&Loans }\end{array}$ & $\begin{array}{c}\text { Security } \\
\text { brokers and } \\
\text { dealers }\end{array}$ & $\begin{array}{c}\text { Total, } \\
\text { per country }\end{array}$ \\
\hline UNITED STATES & 48 & 9 & 18 & 2 & 77 \\
\hline CANADA & 3 & 6 & 6 & 0 & 15 \\
\hline UNITED KINGDOM & 5 & 4 & 5 & 0 & 14 \\
\hline JAPAN & 0 & 0 & 11 & 2 & 13 \\
\hline SOUTH AFRICA & 4 & 3 & 4 & 1 & 12 \\
\hline ITALY & 3 & 3 & 5 & 0 & 11 \\
\hline TURKEY & 4 & 2 & 5 & 0 & 11 \\
\hline AUSTRALIA & 3 & 1 & 4 & 1 & 9 \\
\hline SWITZERLAND & 2 & 4 & 3 & 0 & 9 \\
\hline FRANCE & 1 & 3 & 4 & 0 & 8 \\
\hline GERMANY & 1 & 5 & 2 & 0 & 8 \\
\hline TAIWAN & 4 & 3 & 1 & 0 & 8 \\
\hline INDIA & 0 & 1 & 6 & 0 & 7 \\
\hline INDONESIA & 3 & 3 & 1 & 0 & 7 \\
\hline BERMUDA & 6 & 0 & 0 & 0 & 6 \\
\hline SPAIN & 1 & 1 & 4 & 0 & 6 \\
\hline SINGAPORE & 1 & 2 & 3 & 0 & 6 \\
\hline IRELAND & 2 & 1 & 2 & 0 & 5 \\
\hline HONG KONG & 1 & 2 & 2 & 0 & 5 \\
\hline GREECE & 0 & 1 & 4 & 0 & 5 \\
\hline DENMARK & 2 & 0 & 2 & 0 & 4 \\
\hline SWEDEN & 0 & 0 & 4 & 0 & 4 \\
\hline CHINA & 0 & 0 & 3 & 1 & 4 \\
\hline BELGIUM & 1 & 0 & 2 & 0 & 3 \\
\hline AUSTRIA & 0 & 2 & 1 & 0 & 3 \\
\hline BRAZIL & 0 & 0 & 3 & 0 & 3 \\
\hline NORWAY & 0 & 1 & 1 & 0 & 2 \\
\hline NETHERLANDS & 0 & 1 & 1 & 0 & 2 \\
\hline FINLAND & 1 & 0 & 1 & 0 & 2 \\
\hline PORTUGAL & 0 & 0 & 2 & 0 & 2 \\
\hline NEW ZEALAND & 0 & 2 & 0 & 0 & 2 \\
\hline MEXICO & 0 & 0 & 1 & 0 & 1 \\
\hline RUSSIAN FEDERATION & 0 & 0 & 1 & 0 & 1 \\
\hline Total & 96 & 60 & 112 & 7 & 275 \\
\hline
\end{tabular}

Source: Bloomberg LLP and author's calculations. 
Table 1 (continued). Distribution of firms per country

Panel B: Sample period: Jan. 1, 2005 - Dec. 31 - 2008

\begin{tabular}{|c|c|c|c|c|c|}
\hline \multirow[b]{2}{*}{ Country } & \multicolumn{5}{|c|}{ Type of firm } \\
\hline & $\begin{array}{c}\text { Insurance: } \\
\text { Property and } \\
\text { Casualty; Health }\end{array}$ & $\begin{array}{c}\text { Life } \\
\text { Insurance }\end{array}$ & $\begin{array}{c}\text { Banks and } \\
\text { Savings\&Loans }\end{array}$ & $\begin{array}{c}\text { Security } \\
\text { brokers and } \\
\text { dealers }\end{array}$ & $\begin{array}{c}\text { Total, } \\
\text { per country }\end{array}$ \\
\hline UNITED STATES & 59 & 14 & 18 & 2 & 93 \\
\hline JAPAN & 2 & 1 & 17 & 2 & 22 \\
\hline UNITED KINGDOM & 8 & 4 & 5 & 0 & 17 \\
\hline CANADA & 4 & 6 & 6 & 0 & 16 \\
\hline TAIWAN & 4 & 6 & 4 & 0 & 14 \\
\hline SOUTH AFRICA & 4 & 4 & 4 & 1 & 13 \\
\hline ITALY & 3 & 3 & 6 & 0 & 12 \\
\hline BERMUDA & 11 & 0 & 0 & 0 & 11 \\
\hline TURKEY & 4 & 2 & 5 & 0 & 11 \\
\hline GERMANY & 1 & 5 & 4 & 0 & 10 \\
\hline CHINA & 1 & 2 & 5 & 2 & 10 \\
\hline AUSTRALIA & 3 & 1 & 4 & 1 & 9 \\
\hline SWITZERLAND & 2 & 4 & 3 & 0 & 9 \\
\hline FRANCE & 1 & 3 & 5 & 0 & 9 \\
\hline INDIA & 0 & 1 & 8 & 0 & 9 \\
\hline SPAIN & 1 & 1 & 5 & 0 & 7 \\
\hline IRELAND & 3 & 1 & 2 & 0 & 6 \\
\hline HONG KONG & 1 & 2 & 3 & 0 & 6 \\
\hline SINGAPORE & 1 & 2 & 3 & 0 & 6 \\
\hline INDONESIA & 1 & 2 & 3 & 0 & 6 \\
\hline GREECE & 0 & 1 & 4 & 0 & 5 \\
\hline DENMARK & 2 & 0 & 2 & 0 & 4 \\
\hline SWEDEN & 0 & 0 & 4 & 0 & 4 \\
\hline BRAZIL & 1 & 0 & 3 & 0 & 4 \\
\hline BELGIUM & 1 & 0 & 2 & 0 & 3 \\
\hline AUSTRIA & 0 & 2 & 1 & 0 & 3 \\
\hline NORWAY & 0 & 1 & 1 & 0 & 2 \\
\hline NETHERLANDS & 0 & 1 & 1 & 0 & 2 \\
\hline FINLAND & 1 & 0 & 1 & 0 & 2 \\
\hline PORTUGAL & 0 & 0 & 2 & 0 & 2 \\
\hline NEW ZEALAND & 0 & 2 & 0 & 0 & 2 \\
\hline MEXICO & 0 & 0 & 1 & 0 & 1 \\
\hline RUSSIAN FEDERATION & 0 & 0 & 1 & 0 & 1 \\
\hline Total & 119 & 71 & 133 & 8 & 331 \\
\hline
\end{tabular}

Source: Bloomberg LLP and author's calculations. 
Table 1 (continued). Distribution of firms per country

Panel C: Sample period: Jan. 1, 2009 - Jul. 31 - 2012

\begin{tabular}{|c|c|c|c|c|c|}
\hline \multirow[b]{2}{*}{ Country } & \multicolumn{5}{|c|}{ Type of firm } \\
\hline & $\begin{array}{c}\text { Insurance: } \\
\text { Property and } \\
\text { Casualty; Health }\end{array}$ & $\begin{array}{c}\text { Life } \\
\text { Insurance }\end{array}$ & $\begin{array}{c}\text { Banks and } \\
\text { Savings\&Loans }\end{array}$ & $\begin{array}{c}\text { Security } \\
\text { brokers and } \\
\text { dealers }\end{array}$ & $\begin{array}{c}\text { Total, } \\
\text { per country }\end{array}$ \\
\hline UNITED STATES & 65 & 14 & 18 & 4 & 101 \\
\hline JAPAN & 3 & 2 & 19 & 2 & 26 \\
\hline UNITED KINGDOM & 10 & 7 & 5 & 0 & 22 \\
\hline CHINA & 1 & 3 & 14 & 2 & 20 \\
\hline CANADA & 5 & 6 & 6 & 0 & 17 \\
\hline BERMUDA & 15 & 0 & 0 & 0 & 15 \\
\hline SOUTH AFRICA & 4 & 5 & 4 & 1 & 14 \\
\hline TAIWAN & 4 & 6 & 4 & 0 & 14 \\
\hline ITALY & 3 & 3 & 7 & 0 & 13 \\
\hline TURKEY & 4 & 2 & 5 & 0 & 11 \\
\hline AUSTRALIA & 4 & 1 & 4 & 1 & 10 \\
\hline SWITZERLAND & 3 & 4 & 3 & 0 & 10 \\
\hline GERMANY & 1 & 5 & 4 & 0 & 10 \\
\hline INDIA & 0 & 2 & 8 & 0 & 10 \\
\hline FRANCE & 1 & 3 & 5 & 0 & 9 \\
\hline INDONESIA & 4 & 2 & 3 & 0 & 9 \\
\hline SPAIN & 1 & 1 & 6 & 0 & 8 \\
\hline IRELAND & 3 & 1 & 2 & 0 & 6 \\
\hline HONG KONG & 1 & 2 & 3 & 0 & 6 \\
\hline SINGAPORE & 1 & 2 & 3 & 0 & 6 \\
\hline BRAZIL & 2 & 0 & 4 & 0 & 6 \\
\hline DENMARK & 3 & 0 & 2 & 0 & 5 \\
\hline SWEDEN & 1 & 0 & 4 & 0 & 5 \\
\hline GREECE & 0 & 1 & 4 & 0 & 5 \\
\hline AUSTRIA & 0 & 2 & 2 & 0 & 4 \\
\hline NORWAY & 0 & 2 & 1 & 0 & 3 \\
\hline BELGIUM & 1 & 0 & 2 & 0 & 3 \\
\hline NETHERLANDS & 0 & 1 & 1 & 0 & 2 \\
\hline FINLAND & 1 & 0 & 1 & 0 & 2 \\
\hline PORTUGAL & 0 & 0 & 2 & 0 & 2 \\
\hline RUSSIAN FEDERATION & 0 & 0 & 2 & 0 & 2 \\
\hline NEW ZEALAND & 0 & 2 & 0 & 0 & 2 \\
\hline CAYMAN ISLANDS & 1 & 0 & 0 & 0 & 1 \\
\hline MEXICO & 0 & 0 & 1 & 0 & 1 \\
\hline Total & 142 & 79 & 149 & 10 & 380 \\
\hline
\end{tabular}

Source: Bloomberg LLP and author's calculations. 
Table 1 (continued). Distribution of firms per country

Panel D: Sample period: Jan. 1, 2013 - Jul. 31 - 2016

\begin{tabular}{|c|c|c|c|c|c|}
\hline \multirow[b]{2}{*}{ Country } & \multicolumn{5}{|c|}{ Type of firm } \\
\hline & $\begin{array}{c}\text { Insurance: } \\
\text { Property and } \\
\text { Casualty; Health }\end{array}$ & $\begin{array}{c}\text { Life } \\
\text { Insurance }\end{array}$ & $\begin{array}{l}\text { Banks and } \\
\text { Savings\&Loans }\end{array}$ & $\begin{array}{l}\text { Security } \\
\text { brokers and } \\
\text { dealers }\end{array}$ & $\begin{array}{c}\text { Total, } \\
\text { per country }\end{array}$ \\
\hline UNITED STATES & 65 & 16 & 19 & 4 & 104 \\
\hline JAPAN & 5 & 4 & 20 & 2 & 31 \\
\hline CHINA & 2 & 4 & 17 & 3 & 26 \\
\hline UNITED KINGDOM & 12 & 7 & 5 & 0 & 24 \\
\hline CANADA & 6 & 6 & 6 & 0 & 18 \\
\hline TAIWAN & 4 & 6 & 5 & 0 & 15 \\
\hline BERMUDA & 13 & 0 & 0 & 0 & 13 \\
\hline ITALY & 3 & 3 & 7 & 0 & 13 \\
\hline SOUTH AFRICA & 4 & 4 & 4 & 1 & 13 \\
\hline TURKEY & 4 & 2 & 5 & 0 & 11 \\
\hline AUSTRALIA & 4 & 1 & 4 & 1 & 10 \\
\hline GERMANY & 2 & 4 & 4 & 0 & 10 \\
\hline SWITZERLAND & 3 & 4 & 3 & 0 & 10 \\
\hline INDIA & 0 & 2 & 8 & 0 & 10 \\
\hline SPAIN & 1 & 1 & 7 & 0 & 9 \\
\hline FRANCE & 1 & 3 & 5 & 0 & 9 \\
\hline BRAZIL & 3 & 0 & 4 & 1 & 8 \\
\hline INDONESIA & 3 & 2 & 3 & 0 & 8 \\
\hline HONG KONG & 1 & 3 & 3 & 0 & 7 \\
\hline SINGAPORE & 2 & 2 & 3 & 0 & 7 \\
\hline IRELAND & 3 & 1 & 2 & 0 & 6 \\
\hline GREECE & 1 & 1 & 4 & 0 & 6 \\
\hline DENMARK & 3 & 0 & 2 & 0 & 5 \\
\hline SWEDEN & 1 & 0 & 4 & 0 & 5 \\
\hline NORWAY & 1 & 2 & 1 & 0 & 4 \\
\hline NETHERLANDS & 0 & 3 & 1 & 0 & 4 \\
\hline AUSTRIA & 0 & 2 & 2 & 0 & 4 \\
\hline BELGIUM & 1 & 0 & 2 & 0 & 3 \\
\hline MEXICO & 0 & 0 & 2 & 0 & 2 \\
\hline RUSSIAN FEDERATION & 0 & 0 & 2 & 0 & 2 \\
\hline NEW ZEALAND & 0 & 2 & 0 & 0 & 2 \\
\hline FINLAND & 1 & 0 & 0 & 0 & 1 \\
\hline CAYMAN ISLANDS & 1 & 0 & 0 & 0 & 1 \\
\hline PORTUGAL & 0 & 0 & 1 & 0 & 1 \\
\hline Total & 150 & 85 & 155 & 12 & 402 \\
\hline
\end{tabular}

Source: Bloomberg LLP and author's calculations. 
The calculation of the DY and CLNDY systemic rankings built on the estimation of three VAR specifications differing in the number of lags: 4, 8, and 12. For a given variance decomposition method, the number of lags did not affect much the systemic risk and vulnerability rankings. The Spearman rank correlations ranged from 0.95 to 0.99, and the Kendal rank correlations from 0.85 to 0.90 . Figure 1 shows the distribution of the Pesaran-Shin total GFEVD contribution to the equity returns of single firms generated from a VAR(8) specification. After normalization, these GFEVDs serve to construct the DY network and its systemic rankings. However, the wide dispersion of the total GFEVD, with many observations below one hundredth or above one, makes the interpretation of the DY ranking ambiguous as discussed earlier (Figure 1).

Figure 1. Distribution of Pesaran-Shin total GFEVD contributions to the equity returns of individual firms.

Full sample and sub-sample periods
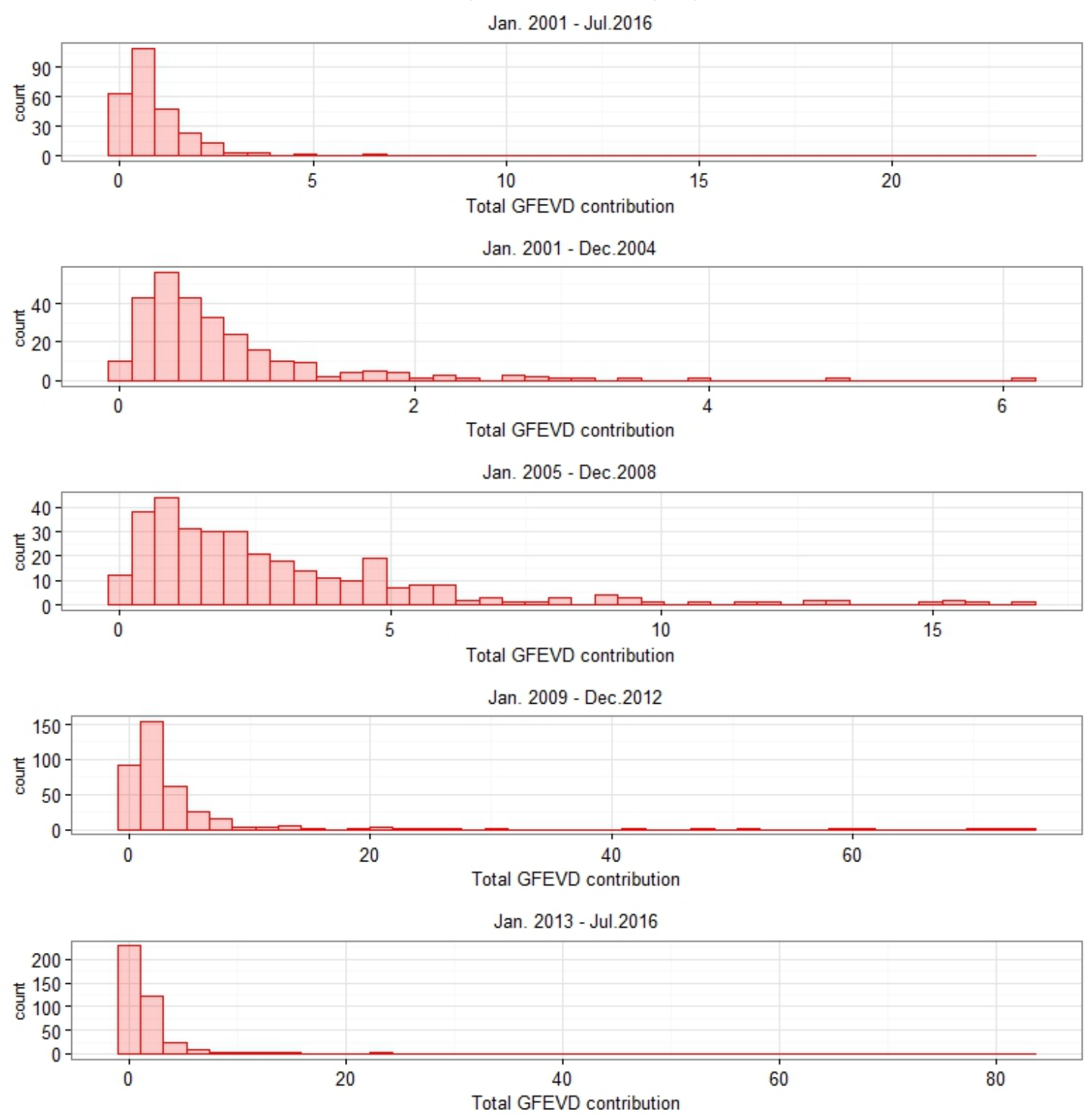

Source: Bloomberg LLP and author's calculations. 
To highlight the differences between the DY and CLNDY systemic rankings, Table 2 presents their nonparametric Spearman and Kendall rank correlations. The low sensitivity of the DY and CLNDY systemic rankings to the lag specification is also evident in the low sensitivity of the cross-systemic ranking correlation across different lags.

Table 2. Rank correlations, Diebold-Yilmaz and corrected Lanne-Nyberg-Diebold-Yilmaz rankings*

\begin{tabular}{|c|c|c|c|c|c|c|}
\hline \multirow{2}{*}{$\begin{array}{l}\text { Correlation measure } \\
\text { Number of lags in VAR }\end{array}$} & \multicolumn{3}{|c|}{ Spearman } & \multicolumn{3}{|c|}{ Kendall } \\
\hline & 4 & 8 & 12 & 4 & 8 & 12 \\
\hline \multicolumn{7}{|c|}{ Panel A: Systemic Risk Rankings } \\
\hline \multicolumn{7}{|c|}{ All firms } \\
\hline Jan. 2001 - Jul. 2016 & 0.72 & 0.74 & 0.74 & 0.54 & 0.56 & 0.56 \\
\hline Jan. 2001 - Dec. 2004 & 0.68 & 0.64 & 0.65 & 0.50 & 0.46 & 0.47 \\
\hline Jan. 2005 - Dec. 2008 & 0.66 & 0.69 & 0.73 & 0.48 & 0.52 & 0.54 \\
\hline Jan. 2009 - Dec. 2012 & 0.74 & 0.77 & 0.73 & 0.56 & 0.59 & 0.54 \\
\hline Jan. 2013 - Jul. 2016 & 0.54 & 0.54 & 0.56 & 0.38 & 0.39 & 0.40 \\
\hline \multicolumn{7}{|c|}{ Top 100 firms in Diebold-Yilmaz network } \\
\hline Jan. 2001 - Jul. 2016 & 0.17 & 0.32 & 0.34 & 0.11 & 0.22 & 0.23 \\
\hline Jan. 2001 - Dec. 2004 & 0.16 & 0.12 & 0.15 & 0.12 & 0.08 & 0.11 \\
\hline Jan. 2005 - Dec. 2008 & 0.17 & 0.29 & 0.28 & 0.12 & 0.20 & 0.20 \\
\hline Jan. 2009 - Dec. 2012 & 0.06 & 0.05 & 0.01 & 0.04 & 0.04 & 0.01 \\
\hline Jan. 2013 - Jul. 2016 & -0.09 & -0.15 & -0.23 & -0.07 & -0.09 & -0.16 \\
\hline \multicolumn{7}{|c|}{ Top 100 firms in corrected Lanne-Nyberg-Diebold-Yilmaz network } \\
\hline Jan. 2001 - Jul. 2016 & 0.24 & 0.42 & 0.44 & 0.16 & 0.29 & 0.30 \\
\hline Jan. 2001 - Dec. 2004 & 0.69 & 0.64 & 0.63 & 0.50 & 0.44 & 0.44 \\
\hline Jan. 2005 - Dec. 2008 & 0.39 & 0.44 & 0.45 & 0.28 & 0.31 & 0.31 \\
\hline Jan. 2009 - Dec. 2012 & 0.10 & 0.22 & 0.04 & 0.07 & 0.15 & 0.03 \\
\hline Jan. 2013 - Jul. 2016 & 0.22 & 0.34 & 0.32 & 0.15 & 0.23 & 0.21 \\
\hline \multicolumn{7}{|c|}{ Panel B: Systemic Vulnerability Rankings } \\
\hline \multicolumn{7}{|c|}{ All firms } \\
\hline Jan. 2001 - Jul. 2016 & 0.80 & 0.82 & 0.79 & 0.63 & 0.63 & 0.62 \\
\hline Jan. 2001 - Dec. 2004 & 0.84 & 0.80 & 0.82 & 0.67 & 0.62 & 0.65 \\
\hline Jan. 2005 - Dec. 2008 & 0.81 & 0.85 & 0.84 & 0.63 & 0.66 & 0.66 \\
\hline Jan. 2009 - Dec. 2012 & 0.81 & 0.80 & 0.77 & 0.63 & 0.62 & 0.59 \\
\hline Jan. 2013 - Jul. 2016 & 0.79 & 0.76 & 0.75 & 0.61 & 0.59 & 0.57 \\
\hline \multicolumn{7}{|c|}{ Top 100 firms in Diebold-Yilmaz network } \\
\hline Jan. 2001 - Jul. 2016 & 0.62 & 0.63 & 0.71 & 0.47 & 0.47 & 0.53 \\
\hline Jan. 2001 - Dec. 2004 & 0.71 & 0.73 & 0.71 & 0.54 & 0.55 & 0.52 \\
\hline Jan. 2005 - Dec. 2008 & 0.54 & 0.27 & 0.15 & 0.41 & 0.20 & 0.11 \\
\hline Jan. 2009 - Dec. 2012 & 0.61 & 0.62 & 0.46 & 0.46 & 0.47 & 0.34 \\
\hline Jan. 2013 - Jul. 2016 & 0.63 & 0.60 & 0.47 & 0.46 & 0.44 & 0.33 \\
\hline \multicolumn{7}{|c|}{ Top 100 firms in corrected Lanne-Nyberg-Diebold-Yilmaz network } \\
\hline Jan. 2001 - Jul. 2016 & 0.78 & 0.77 & 0.79 & 0.59 & 0.58 & 0.61 \\
\hline Jan. 2001 - Dec. 2004 & 0.77 & 0.73 & 0.65 & 0.59 & 0.55 & 0.49 \\
\hline Jan. 2005 - Dec. 2008 & 0.55 & 0.26 & 0.20 & 0.39 & 0.18 & 0.13 \\
\hline Jan. 2009 - Dec. 2012 & 0.62 & 0.51 & 0.43 & 0.45 & 0.36 & 0.30 \\
\hline Jan. 2013 - Jul. 2016 & 0.51 & 0.41 & 0.38 & 0.36 & 0.28 & 0.25 \\
\hline
\end{tabular}

* All correlations significantly different from zero, except in the January 2009 - December 2012 and the January 2013 - July 2016 periods for the top 100 firms in the Diebold-Yilmaz network. Source: Bloomberg LLP and author's calculations. 
Although there is a positive correlation between the DY and CLNDY systemic rankings comprising all firms, the correlation is weaker for risk rankings (Table 2, Panel A) than for vulnerability rankings (Table 2, Panel B). Moreover, the systemic risk ranking correlations dropped in the last subsample period; on the contrary, systemic vulnerability ranking correlations were in line with the values observed in earlier periods or in the full sample period.

The differences in systemic risk rankings introduced by the choice of variance decomposition become more evident in a correlation analysis restricted only to rankings of the top one hundred riskiest firms. In the first exercise, the top one hundred firms correspond to those identified by the DY network. The correlations with the corresponding rankings in the CLNDY network are very low, and in some instances, statistically insignificant. In the second exercise, the top one hundred firms correspond to those identified by the CLNDY network. In this case, the rankings appeared quite correlated in the pre-crisis period of January 2001 to December 2004, but have decoupled since then. As for the top one hundred vulnerable firms, regardless of whether the DY or the CLNDY network guide their selection, rank correlations are higher than for the top one hundred riskiest firms, but they declined sharply in the run up to the Lehman Brothers bankruptcy and global financial crisis during the period of January 2005 - December 2008.

Figure 2, which shows the number of overlapping firms in the DY and CLNDY rankings of the top fifty riskiest and most vulnerable firms, reinforces the analysis. The overlap is considerable for the vulnerability rankings, ranging from 70 to 90 percent depending on the sample period. In the risk rankings, however, the overlap does not exceed 50 percent and has declined in the most recent sub-period. Tables 3 and 4, listing the top firms, provide more details.

Figure 2. Number of overlapping firms in the top fifty DY and CLNDY rankings (ranking based on a VAR(8) specification)

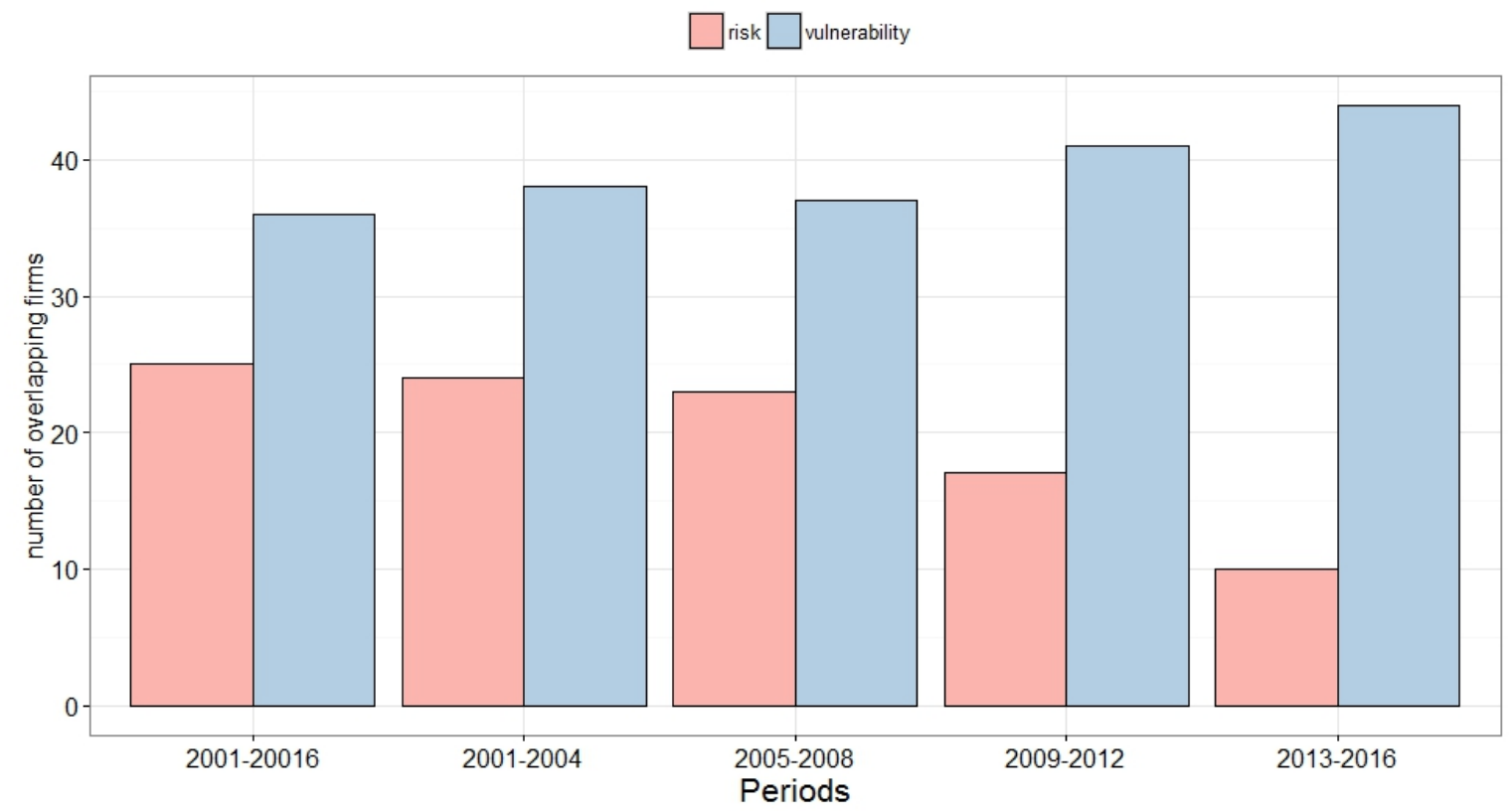

Source: Bloomberg LLP and author's calculations. 
Table 3a. Systemic risk rankings, all firms: January 2001 - December 2004

(ranking based on a VAR(8) specification)

\begin{tabular}{|c|c|c|c|c|}
\hline \multicolumn{5}{|c|}{ Systemic risk ranking } \\
\hline Ranking & Diebold-Yilmaz network & & Corrected Lanne-Nyberg-Diebold-Yilmaz network & \\
\hline 1 & NOVAE GROUPPLC & UNITED KINGDOM & DEUTSCHEBANK AKTIENGESELLSCHAFT & GERMANY \\
\hline 2 & SCOR SE & FRANCE & BANCO BILBAO VIZCAYA ARGENTARIA SA & SPAIN \\
\hline 3 & MEDIOLANUM S.P.A & ITALY & SOCIETE GENERALE & FRANCE \\
\hline 4 & YAPI VE KREDI BANKASI AS & TURKEY & AXA SA & FRANCE \\
\hline 5 & ZURICH INSURANCE GROUP LIMITED & SWTZERLAND & MEDIOLANUM S.P.A & ITALY \\
\hline 6 & AXA SA & FRANCE & BANCO SANTANDER SA & SPAIN \\
\hline 7 & TURKIYE IS BANKASI A.S. & TURKEY & ING GROEP N.V. & NETHERLANDS \\
\hline 8 & SWSS LIFE HOLDING AG & SWTZERLAND & BNP PARIBAS SA & FRANCE \\
\hline 9 & RSA INSURANCE GROUP PLC & UNITED KINGDOM & BANKINTER, S.A. & SPAIN \\
\hline 10 & COMMERZBANK AKTIENGESELLSCHAFT & GERMANY & STANDARD CHARTERED PLC & UNITED KINGDOM \\
\hline 11 & AEGON N.V. & NETHERLANDS & CREDIT SUISSE GROUPAG & SWTZERLAND \\
\hline 12 & ING GROEP N.V. & NETHERLANDS & ALLIANZ SE & GERMANY \\
\hline 13 & GUNES SIGORTA A.S. & TURKEY & MEDIOBANCA SPA & ITALY \\
\hline 14 & ALLIANZ SE & GERMANY & COMMERZBANK AKTIENGESELLSCHAFT & GERMANY \\
\hline 15 & SINAR MAS MULTIARTHA TBK PT & INDONESIA & UBS AG & SWTZERLAND \\
\hline 16 & TURKIYE GARANTI BANKASI A.S. & TURKEY & UNICREDIT SPA & ITALY \\
\hline 17 & DEUTSCHE BANK AKTIENGESELLSCHAFT & GERMANY & UBS GROUP AG & SWTZERLAND \\
\hline 18 & RAY SIGORTA A.S. & TURKEY & PRUDENTIAL PLC & UNITED KINGDOM \\
\hline 19 & MUENCHENER RUCKVERSICHERUNGS-GESELLSCHAF & GERMANY & NORTHERN TRUST CORP & UNITED STATES \\
\hline 20 & CNPASSURANCES & FRANCE & ASSICURAZIONI GENERALI SPA & ITALY \\
\hline 21 & ANADOLU HAYAT EMEKLILIK A.S. & TURKEY & HARTFORD FINANCIAL SERVICES & UNITED STATES \\
\hline 22 & PRUDENTIAL PLC & UNITED KINGDOM & TORCHMARK CORP & UNITED STATES \\
\hline 23 & CREDIT SUISSE GROUPAG & SWTZERLAND & ZURICH INSURANCE GROUP LIMITED & SWTZERLAND \\
\hline 24 & PANIN FINANCIAL TBK PT & INDONESIA & ROYAL BANK OF SCOTLAND GROUP PLC & UNITED KINGDOM \\
\hline 25 & SOCIETE GENERALE & FRANCE & MUENCHENER RUCKVERSICHERUNGS-GESELLSCHAF & GERMANY \\
\hline 26 & HANNOVER RUECK SE & GERMANY & SWEDBANK AB & SWEDEN \\
\hline 27 & AKSIGORTA ANONIM SIRKETI & TURKEY & HSBC HOLDINGS PLC & UNITED KINGDOM \\
\hline 28 & AKBANK TAS & TURKEY & BANCA MONTEDE PASCHI DI SIENA SPA & ITALY \\
\hline 29 & HANOVER INSURANCE GROUP INC & UNITED STATES & SUN LIFE FINANCIAL INC & CANADA \\
\hline 30 & AMLIN PLC & UNITED KINGDOM & MBIA INC & UNITED STATES \\
\hline 31 & BANCO DO BRASIL SA & BRAZIL & LINCOLN NATIONAL CORP & UNITED STATES \\
\hline 32 & BNP PARIBAS SA & FRANCE & KBC GROUP NV & BELGIUM \\
\hline 33 & CHUBB CORP & UNITED STATES & SWSS REAG & SWTZERLAND \\
\hline 34 & BALOISE HOLDING AG & SWTZERLAND & AEGON N.V. & NETHERLANDS \\
\hline 35 & HISCOX PLC & UNITED KINGDOM & DEXIA SA & BELGIUM \\
\hline 36 & BANCO BRADESCO S.A. & BRAZIL & HANNOVER RUECK SE & GERMANY \\
\hline 37 & BANCO BILBAO VIZCAYA ARGENTARIA SA & SPAIN & METLIFE INC & UNITED STATES \\
\hline 38 & HACI OMER SABANCI HOLDING A.S. & TURKEY & CITIGROUPINC & UNITED STATES \\
\hline 39 & PMORGAN CHASE \& CO & UNITED STATES & BALOISE HOLDING AG & SMTZERLAND \\
\hline 40 & DEXIA SA & BELGIUM & STATESTRET CORP & UNITED STATES \\
\hline 41 & BANCO SANTANDER SA & SPAIN & BARCLAYS PLC & UNITED KINGDOM \\
\hline 42 & MORGAN STANLEY & UNITED STATES & PMORGAN CHASE \& CO & UNITED STATES \\
\hline 43 & AVIVA PLC & UNITED KINGDOM & AMLIN PLC & UNITED KINGDOM \\
\hline 44 & ITAU UNIBANCO HOLDING S.A. & BRAZIL & MANULIFE FINANCIAL CORP & CANADA \\
\hline 45 & SWSS REAG & SWTZERLAND & SKANDINAVISKA ENSKILDA BANKEN & SWEDEN \\
\hline 46 & SKANDINAVISKA ENSKILDA BANKEN & SWEDEN & BANCA POPOLARE DELL'EMILIA ROMAGNA SC & ITALY \\
\hline 47 & FEDERATED NATIONAL HLDG CO & UNITED STATES & VITTORIA ASSICURAZIONI SPA & ITALY \\
\hline 48 & INTESA SANPAOLO SPA & ITALY & WELLS FARGO \& CO & UNITED STATES \\
\hline 49 & BANCA MONTE DEI PASCHI DI SIENA SPA & ITALY & OLD MUTUAL PLC & UNITED KINGDOM \\
\hline 50 & STANDARD CHARTERED PLC & UNITED KINGDOM & BANCO COMERCIAL PORTUGUES, S.A. & PORTUGAL \\
\hline
\end{tabular}

Source: Bloomberg LLP and author's calculations. 
Table 3b. Systemic risk rankings, all firms: January 2005 - December 2008

(ranking based on a VAR(8) specification)

\begin{tabular}{|c|c|c|c|c|}
\hline \multicolumn{5}{|c|}{ Systemic risk ranking } \\
\hline Ranking & Diebold-Yilmaz network & & Corrected Lanne-Nyberg-Diebold-Yilmaz network & \\
\hline 1 & AMERICAN INTERNATIONAL GROUP & UNITED STATES & AMERICAN INTERNATIONAL GROUP & UNITED STATES \\
\hline 2 & RADIAN GROUPINC & UNITED STATES & MORGAN STANLEY & UNITED STATES \\
\hline 3 & MBIA INC & UNITED STATES & REGIONS FINANCIAL CORP & UNITED STATES \\
\hline 4 & MGIC INVESTMENT CORP/ M & UNITED STATES & COMERICA INC & UNITED STATES \\
\hline 5 & GENWORTH FINANCIAL INC & UNITED STATES & CHUBB CORP & UNITED STATES \\
\hline 6 & XL GROUP PLC - ADR & IREAND & WELS FARGO \& CO & UNITED STATES \\
\hline 7 & REGIONS FINANCIAL CORP & UNITED STATES & SUNTRUST BANKS INC & UNITED STATES \\
\hline 8 & MORGAN STANLEY & UNITED STATES & HUMANA INC & UNITED STATES \\
\hline 9 & ASSURED GUARANTY LTD & BERMUDA & CNA FINANCIAL CORP & UNITED STATES \\
\hline 10 & HUMANA INC & UNITED STATES & MERCURY GENERAL CORP & UNITED STATES \\
\hline 11 & COMERICA INC & UNITED STATES & DEUTSCHE BANK AKTIENGESELLSCHAFT & GERMANY \\
\hline 12 & PERMANENT TSB GROUP HOLDINGS PLC & IREAND & GENWORTH FINANCIAL INC & UNITED STATES \\
\hline 13 & UTG INC & UNITED STATES & MARKE CORP & UNITED STATES \\
\hline 14 & CITIGROUPINC & UNITED STATES & AMERICAN FINANCIAL GROUPINC & UNITED STATES \\
\hline 15 & ITAU UNIBANCO HOLDING S.A. & BRAZIL & GOLDMAN SACHS GROUPINC & UNITED STATES \\
\hline 16 & ZIONS BANCORPORATION & UNITED STATES & PRUDENTIAL FINANCIAL INC & UNITED STATES \\
\hline 17 & CNO FINANCIAL GROUPINC & UNITED STATES & BANK OF AMERICA CORP & UNITED STATES \\
\hline 18 & HARTFORD FINANCIAL SERVICES & UNITED STATES & ZIONS BANCORPORATION & UNITED STATES \\
\hline 19 & SUNTRUST BANKS INC & UNITED STATES & OLD REPUBLIC INTL CORP & UNITED STATES \\
\hline 20 & RAY SIGORTA A.S. & TURKEY & METLIFE INC & UNITED STATES \\
\hline 21 & BANCO DO BRASIL SA & BRAZIL & ALLSTATE CORP & UNITED STATES \\
\hline 22 & WELLS FARGO \& CO & UNITED STATES & CREDIT SUISSE GROUP AG & SWTZERLAND \\
\hline 23 & BANCO BRADESCO S.A. & BRAZIL & LINCOLN NATIONAL CORP & UNITED STATES \\
\hline 24 & BARCLAYS PLC & UNITED KINGDOM & ALLEGHANY CORP & UNITED STATES \\
\hline 25 & AKSIGORTA ANONIM SIRKETI & TURKEY & XL GROUP PLC - ADR & IREAND \\
\hline 26 & ROYAL BANK OF SCOTLAND GROUP PLC & UNITED KINGDOM & HORACE MANN ĐUUCATORS CORP & UNITED STATES \\
\hline 27 & PRUDENTIAL FINANCIAL INC & UNITED STATES & CITIGROUPINC & UNITED STATES \\
\hline 28 & LINCOLN NATIONAL CORP & UNITED STATES & BB\&T CORP & UNITED STATES \\
\hline 29 & KEYCORP & UNITED STATES & TRAVELERS COS INC & UNITED STATES \\
\hline 30 & TURNERS LTD & NEWZEALAND & KBC GROUP NV & BEGIUM \\
\hline 31 & CREDIT SUISSE GROUPAG & SWTZERLAND & PROASSURANCE CORP & UNITED STATES \\
\hline 32 & MACQUARIE GROUP LIMITED & AUSTRALIA & CNO FINANCIAL GROUPINC & UNITED STATES \\
\hline 33 & GOLDMAN SACHS GROUPINC & UNITED STATES & SUN LIFE FINANCIAL INC & CANADA \\
\hline 34 & STANDARD CHARTERED PLC & UNITED KINGDOM & AXA SA & FRANCE \\
\hline 35 & BANK OF AMERICA CORP & UNITED STATES & ARCH CAPITAL GROUP LTD & BERMUDA \\
\hline 36 & AKBANK TAS & TURKEY & BANCO BILBAO VIZCAYA ARGENTARIA SA & SPAIN \\
\hline 37 & HORACE MANN EDUCATORS CORP & UNITED STATES & KEMPER CORP/ DE & UNITED STATES \\
\hline 38 & TURKIYEIS BANKASI A.S. & TURKEY & BANCO SANTANDER SA & SPAIN \\
\hline 39 & LLOYDS BANKING GROUP PLC & UNITED KINGDOM & EVEREST RE GROUP LTD & BERMUDA \\
\hline 40 & BANK OF IRELAND & IREAND & CANADIAN IMPERIAL BANK & CANADA \\
\hline 41 & SBERBANK ROSSII OAO & RUSSIAN FEDERATIOI & ALLIANZ SE & GERMANY \\
\hline 42 & TURKIYE GARANTI BANKASI A.S. & TURKEY & CINCINNATI FINANCIAL CORP & UNITED STATES \\
\hline 43 & SOCIETE GENERALE & FRANCE & LLOYDS BANKING GROUPPLC & UNITED KINGDOM \\
\hline 44 & ALLIED IRISH BANKS PLC & IREAND & UBS AG & SWTZERLAND \\
\hline 45 & DEUTSCHE BANK AKTIENGESELLSCHAFT & GERMANY & STATEAUTO FINANCIAL CORP & UNITED STATES \\
\hline 46 & STATEAUTO FINANCIAL CORP & UNITED STATES & AEGON N.V. & NETHERLANDS \\
\hline 47 & CNA FINANCIAL CORP & UNITED STATES & U S BANCORP & UNITED STATES \\
\hline 48 & FIFTH THIRD BANCORP & UNITED STATES & UBS GROUPAG & SWTZERLAND \\
\hline 49 & CHALLENGER FINANCIAL SERVICES GROUP LTD & AUSTRALIA & STANDARD CHARTERED PLC & UNITED KINGDOM \\
\hline 50 & AAREAL BANK AG & GERMANY & M \& T BANK CORP & UNITED STATES \\
\hline
\end{tabular}

Source: Bloomberg LLP and author's calculations. 
Table 3c. Systemic risk rankings, all firms: January 2009 - December 2012

(ranking based on a VAR(8) specification)

\begin{tabular}{|c|c|c|c|c|}
\hline \multicolumn{5}{|c|}{ Systemic risk ranking } \\
\hline Ranking & Diebold-Yilmaz network & & Corrected Lanne-Nyberg-Diebold-Yilmaz network & \\
\hline 1 & RADIAN GROUPINC & UNITED STATES & AEGON N.V. & NETHERLANDS \\
\hline 2 & KBC GROUP NV & BELGIUM & ING GROEP N.V. & NETHERLANDS \\
\hline 3 & BANK OF IRELAND & IREAND & ALLIANZ SE & GERMANY \\
\hline 4 & ALLIED IRISH BANKS PLC & IREAND & AFLAC INC & UNITED STATES \\
\hline 5 & MGIC INVESTMENT CORP/ M & UNITED STATES & LOEWS CORP & UNITED STATES \\
\hline 6 & ING GROEP N.V. & NETHERLANDS & SAMPO OYJ & FINLAND \\
\hline 7 & SOCIETE GENERALE & FRANCE & METLIFE INC & UNITED STATES \\
\hline 8 & PHOENIX COMPANIES INC & UNITED STATES & UNUM GROUP & UNITED STATES \\
\hline 9 & BARCLAYS PLC & UNITED KINGDOM & AXA SA & FRANCE \\
\hline 10 & CREDIT AGRICOLE SA & FRANCE & AVIVA PLC & UNITED KINGDOM \\
\hline 11 & AMERICAN INTERNATIONAL GROUP & UNITED STATES & BNP PARIBAS SA & FRANCE \\
\hline 12 & AEGON N.V. & NETHERLANDS & POWER CORP CANADA & CANADA \\
\hline 13 & NATIXIS & FRANCE & HSBC HOLDINGS PLC & UNITED KINGDOM \\
\hline 14 & AAREAL BANK AG & GERMANY & BARCLAYS PLC & UNITED KINGDOM \\
\hline 15 & CNO FINANCIAL GROUPINC & UNITED STATES & CREDIT AGRICOLESA & FRANCE \\
\hline 16 & UNICREDIT SPA & ITALY & POWER FINANCIAL CORP & CANADA \\
\hline 17 & LLOYDS BANKING GROUP PLC & UNITED KINGDOM & SOCIETE GENERALE & FRANCE \\
\hline 18 & ROYAL BANK OF SCOTLAND GROUP PLC & UNITED KINGDOM & DEUTSCHE BANK AKTIENGESELLSCHAFT & GERMANY \\
\hline 19 & GENWORTH FINANCIAL INC & UNITED STATES & HARTFORD FINANCIAL SERVICES & UNITED STATES \\
\hline 20 & COMMERZBANK AKTIENGESELLSCHAFT & GERMANY & BANK OF MONTREAL & CANADA \\
\hline 21 & HARTFORD FINANCIAL SERVICES & UNITED STATES & SKANDINAVISKA ENSKILDA BANKEN & SWEDEN \\
\hline 22 & ERSTE GROUP BANK AG & AUSTRIA & NORDEA BANK AB & SWEDEN \\
\hline 23 & EUROBANK ERGASIAS SA & GREECE & AMERICAN FINANCIAL GROUPINC & UNITED STATES \\
\hline 24 & AXA SA & FRANCE & TORONTO DOMINION BANK & CANADA \\
\hline 25 & BNP PARIBAS SA & FRANCE & COMMONWEALTH BANK OF AUSTRALIA & AUSTRALIA \\
\hline 26 & RAIFFESEN BANK INTERNATIONAL AG & AUSTRIA & PRUDENTIAL FINANCIAL INC & UNITED STATES \\
\hline 27 & ALPHA BANK SA & GREECE & SWSS REAG & SWTZERLAND \\
\hline 28 & INTESA SANPAOLO SPA & ITALY & ZURICH INSURANCE GROUP LIMITED & SWTZERLAND \\
\hline 29 & MBIA INC & UNITED STATES & SVENSKA HANDE SBANKEN AB & SWEDEN \\
\hline 30 & CITIGROUPINC & UNITED STATES & ASSICURAZIONI GENERALI SPA & ITALY \\
\hline 31 & STOREBRAND ASA & NORWAY & TORCHMARK CORP & UNITED STATES \\
\hline 32 & ASSURED GUARANTY LTD & BERMUDA & POHXLA BANK PLC & FINLAND \\
\hline 33 & BANCO POPOLARE & ITALY & BANK OF NOVA SCOTIA & CANADA \\
\hline 34 & REGIONS FINANCIAL CORP & UNITED STATES & CANADIAN IMPERIAL BANK & CANADA \\
\hline 35 & FIFTH THIRD BANCORP & UNITED STATES & LLOYDS BANKING GROUP PLC & UNITED KINGDOM \\
\hline 36 & ZIONS BANCORPORATION & UNITED STATES & NATIONAL AUSTRALIA BANK LIMITED & AUSTRALIA \\
\hline 37 & SKANDINAVISKA ENSKILDA BANKEN & SWEDEN & SWSS LIFE HOLDING AG & SWTZERLAND \\
\hline 38 & BANK OF PIRAEUS S.A. & GREECE & BALOISE HOLDING AG & SWTZERLAND \\
\hline 39 & DEXIA SA & BELGIUM & HELVETIA HOLDING AG & SWTZERLAND \\
\hline 40 & LINCOLN NATIONAL CORP & UNITED STATES & AAREAL BANK AG & GERMANY \\
\hline 41 & DEUTSCHE BANK AKTIENGESELLSCHAFT & GERMANY & ROYAL BANK OF SCOTLAND GROUP PLC & UNITED KINGDOM \\
\hline 42 & BANK OF AMERICA CORP & UNITED STATES & KBC GROUPNV & BElGIUM \\
\hline 43 & UNIPOLSAI ASSICURAZIONI SPA & ITALY & DNB ASA & NORWAY \\
\hline 44 & SWEDBANK AB & SWEDEN & MANULIFE FINANCIAL CORP & CANADA \\
\hline 45 & BANCO BILBAO VIZCAYA ARGENTARIA SA & SPAIN & VIENNA INSURANCE GROUP & AUSTRIA \\
\hline 46 & NATIONAL BANK OF GREECE, S.A. & GREECE & INTESA SANPAOLO SPA & ITALY \\
\hline 47 & AVIVA PLC & UNITED KINGDOM & OLD MUTUAL PLC & UNITED KINGDOM \\
\hline 48 & PERMANENT TSB GROUP HOLDINGS PLC & IREAND & ACKERMANS \& VAN HAAREN & BELGIUM \\
\hline 49 & HUMANA INC & UNITED STATES & ROYAL BANK OF CANADA & CANADA \\
\hline 50 & SUNTRUST BANKS INC & UNITED STATES & ERSTE GROUP BANK AG & AUSTRIA \\
\hline
\end{tabular}

Source: Bloomberg LLP and author's calculations. 
Table 3d. Systemic risk rankings, all firms: January 2013 - July 2016 (ranking based on a VAR(8) specification)

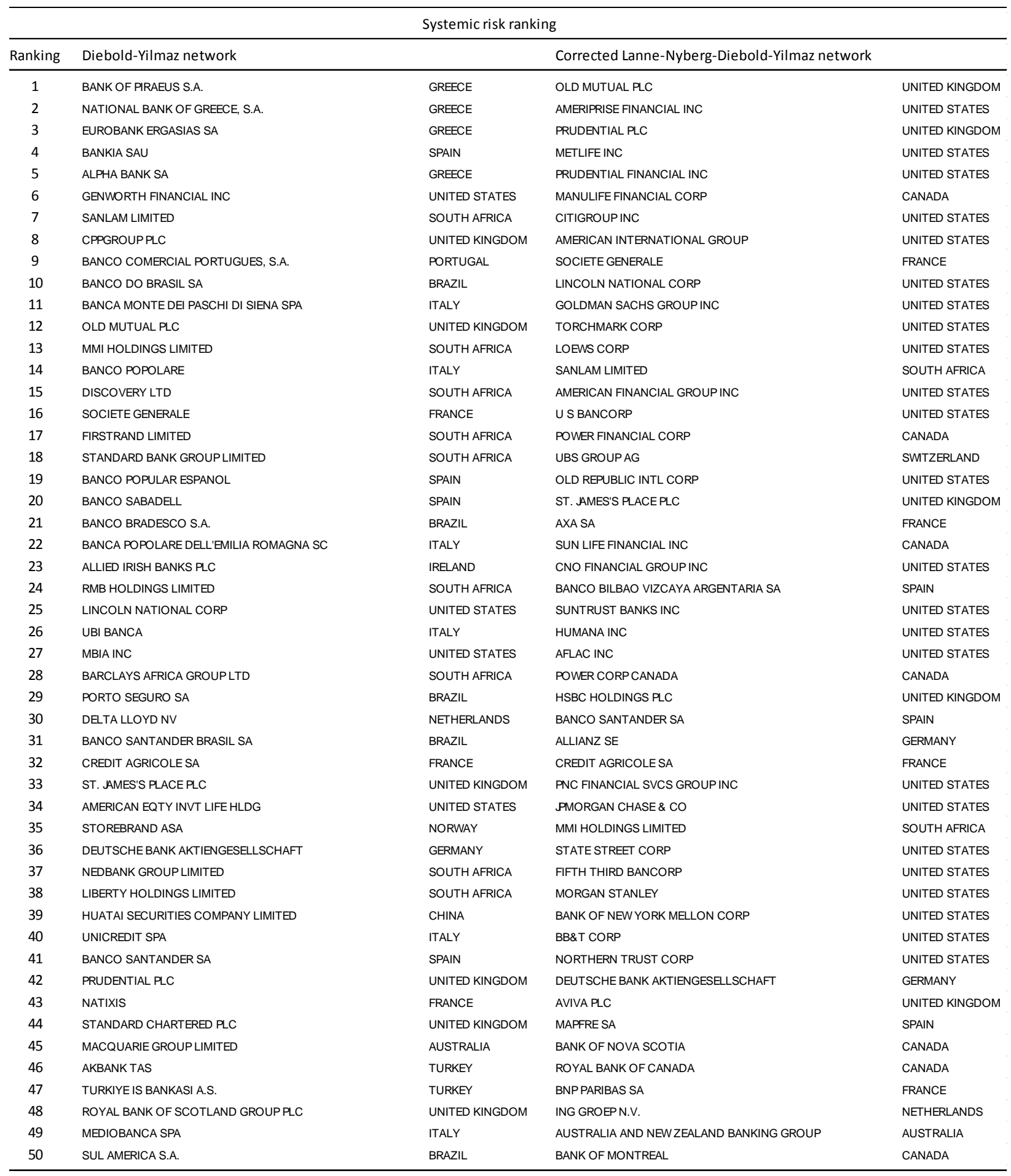

Source: Bloomberg LLP and author's calculations. 
Table 3e. Systemic risk rankings, all firms: January 2001 - July 2016 (ranking based on a VAR(8) specification)

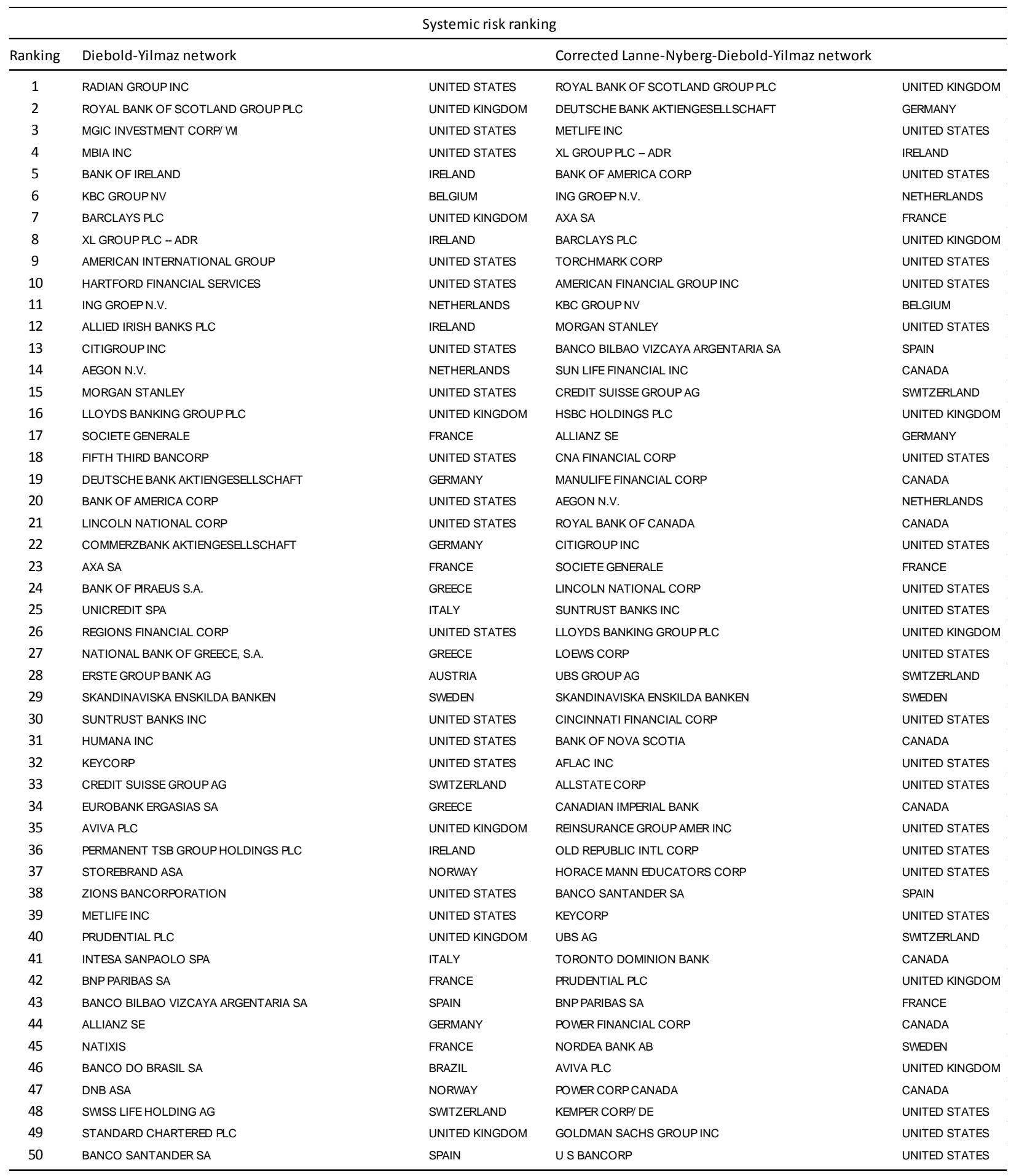

Source: Bloomberg LLP and author's calculations. 
Table 4a. Systemic vulnerability rankings, all firms: January 2001 - December 2004 (ranking based on a VAR(8) specification)

\begin{tabular}{|c|c|c|c|c|}
\hline \multicolumn{5}{|c|}{ Systemic vulnerability ranking } \\
\hline Ranking & Diebold-Yilmaz network & & Corrected Lanne-Nyberg-Diebold-Yilmaz network & \\
\hline 1 & CHINA MINSHENG BANKING CORPORATION LIMITED & CHINA & CHANG HWA COMMERCIAL BANK LIMITED & TAIWAN \\
\hline 2 & CHANG HWA COMMERCIAL BANK LIMITED & TAIWAN & ICICI BANK LIMITED & INDIA \\
\hline 3 & ICICI BANK LIMITED & INDIA & CHINA MINSHENG BANKING CORPORATION LIMITED & CHINA \\
\hline 4 & ASIA FINANCIAL HOLDINGS LTD. & HONG KONG & AXIS BANK LIMITED & INDIA \\
\hline 5 & NATIONAL SEC GROUPINC & UNITED STATES & ASIA FINANCIAL HOLDINGS LTD. & HONG KONG \\
\hline 6 & STATEBANK OF INDIA & INDIA & STATE BANK OF INDIA & INDIA \\
\hline 7 & UNITED OVERSEAS INSURANCE LIMITED & SINGAPORE & ASURANSI BINTANG TBK PT & INDONESIA \\
\hline 8 & PING AN BANK COMPANY LIMITED & CHINA & NATIONAL SEC GROUPINC & UNITED STATES \\
\hline 9 & AXIS BANK LIMITED & INDIA & ADITYA BIRLA NUVO LIMITED & INDIA \\
\hline 10 & INDEQUITY GROUP LIMITED & SOUTH AFRICA & TAIWAN LIFE INSURANCE COMPANY LIMITED & TAIWAN \\
\hline 11 & TAIWAN LIFE INSURANCE COMPANY LIMITED & TAIWAN & PING AN BANK COMPANY LIMITED & CHINA \\
\hline 12 & HAITONG SECURITIES CO. LIMITED & CHINA & INDEQUITY GROUP LIMITED & SOUTH AFRICA \\
\hline 13 & SHANGHAI PUDONG DEVELOPMENT BANK & CHINA & CHINA LIFE INSURANCE COMPANY LIMITED & TAIWAN \\
\hline 14 & ASURANSI BINTANG TBK PT & INDONESIA & FIRST INSURANCE COMPANY LIMITED (THE) & TAIWAN \\
\hline 15 & ADITYA BIRLA NUVO LIMITED & INDIA & PANINVEST TBK PT & INDONESIA \\
\hline 16 & CENTRAL REINSURANCE CORPORATION & TAIWAN & PANIN FINANCIAL TBK PT & INDONESIA \\
\hline 17 & BANCA POPOLARE DELL'EMILIA ROMAGNA SC & ITALY & HAITONG SECURITIES CO. LIMITED & CHINA \\
\hline 18 & NUERNBERGER BETELIGUNGS-AG & GERMANY & SHANGHAI PUDONG DEVEIOPMENT BANK & CHINA \\
\hline 19 & FIRST INSURANCE COMPANY LIMITED (THE) & TAIWAN & GRUPO FINANCIERO BANORTES.A.B. DE C.V. & MEXICO \\
\hline 20 & NAVIGATORS GROUPINC & UNITED STATES & NAVIGATORS GROUPINC & UNITED STATES \\
\hline 21 & HCC INSURANCE HOLDINGS INC & UNITED STATES & ANADOLU ANONIM TURK SIGORTA SIRKETI & TURKEY \\
\hline 22 & GRUPO CATALANA OCCIDENTESA & SPAIN & YAPI VE KREDI BANKASI AS & TURKEY \\
\hline 23 & ANADOLU ANONIM TURK SIGORTA SIRKETI & TURKEY & CHINA TAIPING INSURANCE HOLDINGS COMPANY LIMITED & HONG KONG \\
\hline 24 & UNIQA INSURANCE GROUPAG & AUSTRIA & CENTRAL RENSURANCE CORPORATION & TAIWAN \\
\hline 25 & FIRST ACCEPTANCE CORP & UNITED STATES & EMC INSURANCE GROUPINC & UNITED STATES \\
\hline 26 & WURTTEMBERGISCHELEBENSVERSICHERUNG AG & GERMANY & MEADOWBROOK INS GROUP INC & UNITED STATES \\
\hline 27 & CHINA LIFE INSURANCE COMPANY LIMITED & TAIWAN & MIN XIN HOLDINGS LTD. & HONG KONG \\
\hline 28 & TURNERS LTD & NEW ZEALAND & SBERBANK ROSSII OAO & RUSSIAN FEDERATII \\
\hline 29 & TAIWAN FIRE \& MARINE INSURANCE COMPANY L & TAIWAN & FIRST ACCEPTANCE CORP & UNITED STATES \\
\hline 30 & KANSAS CITY LIFE INS CO & UNITED STATES & MASKAPAI REASURANSI INDONESIA TBK PT & INDONESIA \\
\hline 31 & POHDLA BANK PLC & FINLAND & NUERNBERGER BETELIGUNGS-AG & GERMANY \\
\hline 32 & HANG SENG BANK LIMITED & HONG KONG & BANK OF INDIA & INDIA \\
\hline 33 & WHITE MTNS INS GROUP LTD & BERMUDA & BANK OF BARODA & INDIA \\
\hline 34 & HDFC BANK LIMITED & INDIA & UNITED OVERSEAS INSURANCE LIMITED & SINGAPORE \\
\hline 35 & AMERICAN NATIONAL INSURANCE & UNITED STATES & WURTTEMBERGISCHE LEBENSVERSICHERUNG AG & GERMANY \\
\hline 36 & CHINA TAIPING INSURANCE HOLDINGS COMPANY LIMITED & HONG KONG & TAIWAN FIRE \& MARINE INSURANCE COMPANY L & TAIWAN \\
\hline 37 & NATIONAL WESTERN LIFE-CL A & UNITED STATES & ASURANSI DAYIN MITRA TBK PT & INDONESIA \\
\hline 38 & RENAISSANCERE HOLDINGS LTD & BERMUDA & HCC INSURANCE HOLDINGS INC & UNITED STATES \\
\hline 39 & MIN XIN HOLDINGS LTD. & HONG KONG & TURNERS LTD & NEW ZEALAND \\
\hline 40 & EMC INSURANCE GROUPINC & UNITED STATES & TOWER LIMITED & NEW ZEALAND \\
\hline 41 & MERCURY GENERAL CORP & UNITED STATES & SELECTIVEINS GROUPINC & UNITED STATES \\
\hline 42 & WESTPAC BANKING CORPORATION & AUSTRALIA & HDFC BANK LIMITED & INDIA \\
\hline 43 & VAUDOISEASSURANCES HOLDING & SWTZERLAND & VAUDOISE ASSURANCES HOLDING & SWTZERLAND \\
\hline 44 & KEMPER CORP/ DE & UNITED STATES & UNIQA INSURANCE GROUPAG & AUSTRIA \\
\hline 45 & COMMONWEALTH BANK OF AUSTRALIA & AUSTRALIA & BANCA POPOLARE DELL'EMILIA ROMAGNA SC & ITALY \\
\hline 46 & SEECTIVEINS GROUPINC & UNITED STATES & CITIZENS INC & UNITED STATES \\
\hline 47 & MARKEL CORP & UNITED STATES & RENAISSANCERE HOLDINGS LTD & BERMUDA \\
\hline 48 & ALLEGHANY CORP & UNITED STATES & KANSAS CITY LIFE INS CO & UNITED STATES \\
\hline 49 & PERSONAL GROUP HOLDINGS PLC & UNITED KINGDOM & WHITE MTNS INS GROUP LTD & BERMUDA \\
\hline 50 & FIFTH THIRD BANCORP & UNITED STATES & NATIONAL WESTERN LIFE -CL A & UNITED STATES \\
\hline
\end{tabular}

Source: Bloomberg LLP and author's calculations. 
Table 4b. Systemic vulnerability rankings, all firms: January 2005 - December 2008 (rankings based on a VAR(8) specification)

\begin{tabular}{|c|c|c|c|c|}
\hline \multicolumn{5}{|c|}{ Systemic vulnerability ranking } \\
\hline Ranking & Diebold-Yilmaz network & & Corrected Lanne-Nyberg-Diebold-Yilmaz network & \\
\hline 1 & SHINKONG INSURANCE CO LTD & TAIWAN & SHINKONG INSURANCE CO LTD & TAIWAN \\
\hline 2 & CHANG HWA COMMERCIAL BANK LIMITED & TAIWAN & CHANG HWA COMMERCIAL BANK LIMITED & TAIWAN \\
\hline 3 & ADITYA BIRLA NUVO LIMITED & INDIA & ADITYA BIRLA NUVO LIMITED & INDIA \\
\hline 4 & PUNJAB NATIONAL BANK LTD & INDIA & PUNJAB NATIONAL BANK LTD & INDIA \\
\hline 5 & CATHAY FINANCIAL HOLDING COMPANY LIMITED & TAIWAN & ICICI BANK LIMITED & INDIA \\
\hline 6 & ICICI BANK LIMITED & INDIA & BANK OF BARODA & INDIA \\
\hline 7 & FIRST FINANCIAL HOLDING COMPANY LIMITED & TAIWAN & CATHAY FINANCIAL HOLDING COMPANY LIMITED & TAIWAN \\
\hline 8 & BANK OF BARODA & INDIA & CANARA BANK & INDIA \\
\hline 9 & CANARA BANK & INDIA & WURTTEMBERGISCHE LEBENSVERSICHERUNG AG & GERMANY \\
\hline 10 & INDEQUITY GROUP LIMITED & SOUTH AFRICA & FIRST FINANCIAL HOLDING COMPANY LIMITED & TAIWAN \\
\hline 11 & WURTTEMBERGISCHE LEBENSVERSICHERUNG AG & GERMANY & INDEQUITY GROUP LIMITED & SOUTH AFRICA \\
\hline 12 & HUA NAN FINANCIAL HOLDINGS COMPANY LIMIT & TAIWAN & AXIS BANK LIMITED & INDIA \\
\hline 13 & MEGA FINANCIAL HOLDING COMPANY LIMITED & TAIWAN & SBERBANK ROSSII OAO & RUSSIA \\
\hline 14 & FUBON FINANCIAL HOLDING COMPANY LIMITED & TAIWAN & SHIN KONG FINANCIAL HOLDING COMPANY LIMI & TAIWAN \\
\hline 15 & HDFC BANK LIMITED & INDIA & HDFC BANK LIMITED & INDIA \\
\hline 16 & AXIS BANK LIMITED & INDIA & CHINA LIFE INSURANCE COMPANY LIMITED & TAIWAN \\
\hline 17 & SHIN KONG FINANCIAL HOLDING COMPANY LIMI & TAIWAN & FUBON FINANCIAL HOLDING COMPANY LIMITED & TAIWAN \\
\hline 18 & CENTRAL RENSURANCE CORPORATION & TAIWAN & HUA NAN FINANCIAL HOLDINGS COMPANY LIMIT & TAIWAN \\
\hline 19 & CHINA LIFE INSURANCE COMPANY LIMITED & TAIWAN & MEGA FINANCIAL HOLDING COMPANY LIMITED & TAIWAN \\
\hline 20 & INVESTEC LIMITED & SOUTH AFRICA & UTG INC & UNITED STATES \\
\hline 21 & STATE BANK OF INDIA & INDIA & ARGO GROUP INTL HOLDINGS LTD & BERMUDA \\
\hline 22 & NUERNBERGER BETEILIGUNGS-AG & GERMANY & STATE BANK OF INDIA & INDIA \\
\hline 23 & TAIWAN FIRE \& MARINE INSURANCE COMPANY L & TAIWAN & GRUPO FINANCIERO BANORTES.A.B. DE C.V. & MEXICO \\
\hline 24 & FIRST INSURANCE COMPANY LIMITED (THE) & TAIWAN & BANK OF INDIA & INDIA \\
\hline 25 & GREAT EASTERN HOLDINGS LTD & SINGAPORE & CENTRAL RENSURANCE CORPORATION & TAIWAN \\
\hline 26 & TAIWAN LIFE INSURANCE COMPANY LIMITED & TAIWAN & UNION INSURANCE COMPANY LIMITED & TAIWAN \\
\hline 27 & NOVAE GROUP PLC & UNITED KINGDOM & FIRST INSURANCE COMPANY LIMITED (THE) & TAIWAN \\
\hline 28 & ARGO GROUP INTL HOLDINGS LTD & BERMUDA & WELLCARE HEALTH PLANS INC & UNITED STATES \\
\hline 29 & GRUPO FINANCIERO BANORTE S.A.B. DEC.V. & MEXICO & TAIWAN LIFE INSURANCE COMPANY LIMITED & TAIWAN \\
\hline 30 & UNITED OVERSEAS INSURANCE LIMITED & SINGAPORE & TAIWAN FIRE \& MARINE INSURANCE COMPANY L & TAIWAN \\
\hline 31 & BANK OF INDIA & INDIA & INVESTEC LIMITED & SOUTH AFRICA \\
\hline 32 & CHUGOKU BANK LIMITED (THE) & JAPAN & CITIC SECURITIES CO., LTD. & CHINA \\
\hline 33 & SINGAPORE RENSURANCE CORPORATION LIMITE & SINGAPORE & DREAM INCUBATOR INCORPORATED & JAPAN \\
\hline 34 & PERSONAL GROUP HOLDINGS PLC & UNITED KINGDOM & RAY SIGORTA A.S. & TURKEY \\
\hline 35 & GLOBAL INDEMNITY PLC-ADR & IREAND & PANINVEST TBK PT & INDONESIA \\
\hline 36 & CHESNARA PLC & UNITED KINGDOM & NOVAE GROUP PLC & UNITED KINGDOM \\
\hline 37 & E-L FINANCIAL CORP LTD & CANADA & ATLANTIC AMERICAN CORP & UNITED STATES \\
\hline 38 & SBERBANK ROSSII OAO & RUSSIAN FEDERATION & PING AN BANK COMPANY LIMITED & CHINA \\
\hline 39 & HISCOXPLC & UNITED KINGDOM & TURNERS LTD & NEWZEALAND \\
\hline 40 & VITTORIA ASSICURAZIONI SPA & ITALY & PING AN INSURANCE (GROUP) COMPANY OF CHINA LTD & CHINA \\
\hline 41 & UNICO AMERICAN CORP & UNITED STATES & GLOBAL INDEMNITY PLC-ADR & IRELAND \\
\hline 42 & TRAVELERS COS INC & UNITED STATES & NUERNBERGER BETELIGUNGS-AG & GERMANY \\
\hline 43 & INFINITY PROPERTY \& CAS CORP & UNITED STATES & CENTENE CORP & UNITED STATES \\
\hline 44 & PANINVEST TBK PT & INDONESIA & ASIA FINANCIAL HOLDINGS LTD. & HONG KONG \\
\hline 45 & PING AN INSURANCE (GROUP) COMPANY OF CHINA LTD & CHINA & PICC PROPERTY AND CASUALTY COMPANY LTD & CHINA \\
\hline 46 & VAUDOISE ASSURANCES HOLDING & SWTZERLAND & PANIN FINANCIAL TBK PT & INDONESIA \\
\hline 47 & ASIA FINANCIAL HOLDINGS LTD. & HONG KONG & KINGSTONE COS INC & UNITED STATES \\
\hline 48 & PICC PROPERTY AND CASUALTY COMPANY LTD & CHINA & SHANGHAI PUDONG DEVELOPMENT BANK & CHINA \\
\hline 49 & LIBERTY HOLDINGS LIMITED & SOUTH AFRICA & FEDERATED NATIONAL HLDG CO & UNITED STATES \\
\hline 50 & UNION INSURANCE COMPANY LIMITED & TAIWAN & HISCOXPLC & UNITED KINGDOM \\
\hline
\end{tabular}

Source: Bloomberg LLP and author's calculations. 
Table 4c. Systemic vulnerability rankings, all firms: January 2009 - December 2012 (rankings based on a VAR(8) specification)

\begin{tabular}{|c|c|c|c|c|}
\hline \multicolumn{5}{|c|}{ Systemic vulnerability ranking } \\
\hline Ranking & Diebold-Yilmaz network & & Corrected Lanne-Nyberg-Diebold-Yilmaz network & \\
\hline 1 & FUBON FINANCIAL HOLDING COMPANY LIMITED & TAIWAN & FUBON FINANCIAL HOLDING COMPANY LIMITED & TAIWAN \\
\hline 2 & HDFC BANK LIMITED & INDIA & HDFC BANK LIMITED & INDIA \\
\hline 3 & SHINKONG INSURANCE CO LTD & TAIWAN & SHINKONG INSURANCE CO LTD & TAIWAN \\
\hline 4 & CHANG HWA COMMERCIAL BANK LIMITED & TAIWAN & JSC VTB BANK & RUSSIA \\
\hline 5 & SSC VTB BANK & RUSSIA & CHANG HWA COMMERCIAL BANK LIMITED & TAIWAN \\
\hline 6 & SHIN KONG FINANCIAL HOLDING COMPANY LIMI & TAIWAN & SHIN KONG FINANCIAL HOLDING COMPANY LIMI & TAIWAN \\
\hline 7 & HUA NAN FINANCIAL HOLDINGS COMPANY LIMIT & TAIWAN & STATE BANK OF INDIA & INDIA \\
\hline 8 & MEGA FINANCIAL HOLDING COMPANY LIMITED & TAIWAN & CHINA LIFE INSURANCE COMPANY LIMITED & TAIWAN \\
\hline 9 & STATE BANK OF INDIA & INDIA & BANK OF INDIA & INDIA \\
\hline 10 & CATHAY FINANCIAL HOLDING COMPANY LIMITED & TAIWAN & TAIWAN LIFE INSURANCE COMPANY LIMITED & TAIWAN \\
\hline 11 & CHINA LIFE INSURANCE COMPANY LIMITED & TAIWAN & HUA NAN FINANCIAL HOLDINGS COMPANY LIMIT & TAIWAN \\
\hline 12 & FIRST FINANCIAL HOLDING COMPANY LIMITED & TAIWAN & MEGA FINANCIAL HOLDING COMPANY LIMITED & TAIWAN \\
\hline 13 & TAIWAN LIFE INSURANCE COMPANY LIMITED & TAIWAN & CATHAY FINANCIAL HOLDING COMPANY LIMITED & TAIWAN \\
\hline 14 & BANK OF INDIA & INDIA & FIRST FINANCIAL HOLDING COMPANY LIMITED & TAIWAN \\
\hline 15 & UNICO AMERICAN CORP & UNITED STATES & PUNJAB NATIONAL BANK LTD & INDIA \\
\hline 16 & GUNMA BANK LTD & JAPAN & CANARA BANK & INDIA \\
\hline 17 & PUNJAB NATIONAL BANK LTD & INDIA & AXIS BANK LIMITED & INDIA \\
\hline 18 & CENTRAL REINSURANCE CORPORATION & TAIWAN & BAJAJFINSERV LIMITED & INDIA \\
\hline 19 & AXIS BANK LIMITED & INDIA & INDEQUITY GROUP LIMITED & SOUTH AFRICA \\
\hline 20 & CANARA BANK & INDIA & CENTRAL RENSURANCE CORPORATION & TAIWAN \\
\hline 21 & FIRST INSURANCE COMPANY LIMITED (THE) & TAIWAN & UNICO AMERICAN CORP & UNITED STATES \\
\hline 22 & INDEQUITY GROUP LIMITED & SOUTH AFRICA & GUNMA BANK LTD & JAPAN \\
\hline 23 & BANK OF BARODA & INDIA & ADITYA BIRLA NUVO LIMITED & INDIA \\
\hline 24 & ADITYA BIRLA NUVO LIMITED & INDIA & NATIONAL SEC GROUP INC & UNITED STATES \\
\hline 25 & ICICI BANK LIMITED & INDIA & FIRST INSURANCE COMPANY LIMITED (THE) & TAIWAN \\
\hline 26 & BAJAJFINSERV LIMITED & INDIA & ICICI BANK LIMITED & INDIA \\
\hline 27 & TAIWAN FIRE \& MARINE INSURANCE COMPANY L & TAIWAN & BANK OF BARODA & INDIA \\
\hline 28 & NATIONAL SEC GROUPINC & UNITED STATES & UNION INSURANCE COMPANY LIMITED & TAIWAN \\
\hline 29 & HACHIJNI BANK LIMITED (THE) & JAPAN & KINGSWAY FINANCIAL SVCS INC & CANADA \\
\hline 30 & RESONA HOLDINGS INC & JAPAN & RESONA HOLDINGS INC & JAPAN \\
\hline 31 & UNION INSURANCE COMPANY LIMITED & TAIWAN & TAIWAN FIRE\& MARINE INSURANCE COMPANY L & TAIWAN \\
\hline 32 & CHIBA BANK LTD (THE) & JAPAN & UNIVERSAL AMERICAN CORP & UNITED STATES \\
\hline 33 & 77 BANK LIMITED (THE) & JAPAN & ASURANSI MULTI ARTHA GUNA TBK PT & INDONESIA \\
\hline 34 & DYO BANK LIMITED (THE) & JAPAN & GRUPO FINANCIERO BANORTES.A.B. DE C.V. & MEXICO \\
\hline 35 & RANDALL AND QUILTER INVESTMENT HOLDINGS & UNITED KINGDOM & 77 BANK LIMITED (THE) & JAPAN \\
\hline 36 & UNITED OVERSEAS INSURANCE LIMITED & SINGAPORE & HACHIUNI BANK LIMITED (THE) & JAPAN \\
\hline 37 & ECHEON FINANCIAL HLDGS INC & CANADA & WURTTEMBERGISCHE LEBENSVERSICHERUNG AG & GERMANY \\
\hline 38 & NISHI-NIPPON CITY BANK LTD & JAPAN & INSPLANET AB & SWEDEN \\
\hline 39 & HIROSHIMA BANK LIMITED (THE) & JAPAN & MIZUHO FINANCIAL GROUPINC & JAPAN \\
\hline 40 & MIZUHO FINANCIAL GROUPINC & JAPAN & PERMANENT TSB GROUP HOLDINGS PLC & IRELAND \\
\hline 41 & HANSARD GLOBAL PLC & UNITED KINGDOM & CHIBA BANK LTD (THE) & JAPAN \\
\hline 42 & BANK OF BEIJNG CO., LIMITED. & CHINA & ECHEOON FINANCIAL HLDGS INC & CANADA \\
\hline 43 & SHANGHAI PUDONG DEVELOPMENT BANK & CHINA & BANK OF BEIJNG CO., LIMITED. & CHINA \\
\hline 44 & WUESTENROT \& WUERTTEMBERGISCHEAKTIENGES & GERMANY & SHANGHAI PUDONG DEVELOPMENT BANK & CHINA \\
\hline 45 & CHUGOKU BANK LIMITED (THE) & JAPAN & TURNERS LTD & NEW ZEALAND \\
\hline 46 & ALLEGHANY CORP & UNITED STATES & HANSARD GLOBAL PLC & UNITED KINGDOM \\
\hline 47 & CHINA MINSHENG BANKING CORPORATION LIMITED & CHINA & NISHI-NIPPON CITY BANK LTD & JAPAN \\
\hline 48 & FUKUOKA FINANCIAL GROUP, INC & JAPAN & SBERBANK ROSSII OAO & RUSSIA \\
\hline 49 & SHIZUOKA BANK LTD (THE) & JAPAN & CNPASSURANCES & FRANCE \\
\hline 50 & GRUPO FINANCIERO BANORTES.A.B. DE C.V. & MEXICO & RANDALL AND QUILTER INVESTMENT HOLDINGS & UNITED KINGDOM \\
\hline
\end{tabular}

Source: Bloomberg LLP and author's calculations. 
Table 4d. Systemic vulnerability rankings, all firms: January 2013 - July 2016 (rankings based on a $\operatorname{VAR}(8)$ specification)

\begin{tabular}{|c|c|c|c|c|}
\hline \multicolumn{5}{|c|}{ Systemic vulnerability ranking } \\
\hline \multirow{2}{*}{$\frac{\text { Ranking }}{1}$} & \multicolumn{2}{|l|}{ Diebold-Yilmaz network } & \multicolumn{2}{|l|}{ Corrected Lanne-Nyberg-Diebold-Yilmaz network } \\
\hline & TAIWAN COOPERATIVE BANK & TAIWAN & CPPGROUP PLC & UNITED KINGDOM \\
\hline 2 & TAIWAN FIRE \& MARINE INSURANCE COMPANY L & TAIWAN & TAIWAN COOPERATIVEBANK & TAIWAN \\
\hline 3 & SHIN KONG FINANCIAL HOLDING COMPANY LIMI & TAIWAN & TAIWAN FIRE \& MARINE INSURANCE COMPANY L & TAIWAN \\
\hline 4 & CHINA LIFE INSURANCE COMPANY LIMITED & TAIWAN & SHIN KONG FINANCIAL HOLDING COMPANY LIMI & TAIWAN \\
\hline 5 & CPPGROUP PLC & UNITED KINGDOM & CHINA LIFE INSURANCE COMPANY LIMITED & TAIWAN \\
\hline 6 & SHINKONG INSURANCE CO LTD & TAIWAN & INDEQUITY GROUP LIMITED & SOUTH AFRICA \\
\hline 7 & MERCURIES LIFE INSURANCE CO LTD & TAIWAN & JSC VTB BANK & RUSSIA \\
\hline 8 & INDEQUITY GROUP LIMITED & SOUTH AFRICA & BANK OF INDIA & INDIA \\
\hline 9 & FUBON FINANCIAL HOLDING COMPANY LIMITED & TAIWAN & STATE BANK OF INDIA & INDIA \\
\hline 10 & STATE BANK OF INDIA & INDIA & SHINKONG INSURANCE CO LTD & TAIWAN \\
\hline 11 & FIRST FINANCIAL HOLDING COMPANY LIMITED & TAIWAN & AXIS BANK LIMITED & INDIA \\
\hline 12 & SC VTB BANK & RUSSIA & PUNJAB NATIONAL BANK LTD & INDIA \\
\hline 13 & FIRST INSURANCE COMPANY LIMITED (THE) & TAIWAN & FUBON FINANCIAL HOLDING COMPANY LIMITED & TAIWAN \\
\hline 14 & CENTRAL REINSURANCE CORPORATION & TAIWAN & FBD HOLDINGS PLC & IRELAND \\
\hline 15 & MEGA FINANCIAL HOLDING COMPANY LIMITED & TAIWAN & CANARA BANK & INDIA \\
\hline 16 & FBD HOLDINGS PLC & IREAND & MERCURIES LIFE INSURANCE CO LTD & TAIWAN \\
\hline 17 & HUA NAN FINANCIAL HOLDINGS COMPANY LIMIT & TAIWAN & FIRST INSURANCE COMPANY LIMITED (THE) & TAIWAN \\
\hline 18 & BANK OF INDIA & INDIA & UNION INSURANCE COMPANY LIMITED & TAIWAN \\
\hline 19 & AXIS BANK LIMITED & INDIA & MEGA FINANCIAL HOLDING COMPANY LIMITED & TAIWAN \\
\hline 20 & HDFC BANK LIMITED & INDIA & HDFC BANK LIMITED & INDIA \\
\hline 21 & UNION INSURANCE COMPANY LIMITED & TAIWAN & LIFENET INSURANCE COMPANY & JAPAN \\
\hline 22 & PUNJAB NATIONAL BANK LTD & INDIA & BANK OF BARODA & INDIA \\
\hline 23 & CANARA BANK & INDIA & FIRST FINANCIAL HOLDING COMPANY LIMITED & TAIWAN \\
\hline 24 & UNITED OVERSEAS INSURANCE LIMITED & SINGAPORE & ADITYA BIRLA NUVO LIMITED & INDIA \\
\hline 25 & CHANG HWA COMMERCIAL BANK LIMITED & TAIWAN & PHOENIX COMPANIES INC & UNITED STATES \\
\hline 26 & INDEPENDENCE HOLDING CO & UNITED STATES & LIPPO GENERAL INSURANCE TBK PT & INDONESIA \\
\hline 27 & CATHAY FINANCIAL HOLDING COMPANY LIMITED & TAIWAN & BAJJFINSERV LIMITED & INDIA \\
\hline 28 & WHITE MTNS INS GROUP LTD & BERMUDA & INDEPENDENCE HOLDING CO & UNITED STATES \\
\hline 29 & LIFENET INSURANCE COMPANY & JAPAN & CENTRAL REINSURANCE CORPORATION & TAIWAN \\
\hline 30 & ADITYA BIRLA NUVO LIMITED & INDIA & HUA NAN FINANCIAL HOLDINGS COMPANY LIMIT & TAIWAN \\
\hline 31 & NUERNBERGER BETEILIGUNGS-AG & GERMANY & FEDERATED NATIONAL HLDG CO & UNITED STATES \\
\hline 32 & BAJAJFINSERV LIMITED & INDIA & CATHAY FINANCIAL HOLDING COMPANY LIMITED & TAIWAN \\
\hline 33 & WUESTENROT \& WUERTTEMBERGISCHE AKTIENGES & GERMANY & FIRST ACCEPTANCE CORP & UNITED STATES \\
\hline 34 & KINGSTONECOS INC & UNITED STATES & KINGSTONECOS INC & UNITED STATES \\
\hline 35 & GREAT EASTERN HOLDINGS LTD & SINGAPORE & ICICI BANK LIMITED & INDIA \\
\hline 36 & CREDIT INDUSTRIE ET COMMERCIAL SA & FRANCE & UNITED OVERSEAS INSURANCE LIMITED & SINGAPORE \\
\hline 37 & RANDALL AND QUILTER INVESTMENT HOLDINGS & UNITED KINGDOM & RANDALL AND QUILTER INVESTMENT HOLDINGS & UNITED KINGDOM \\
\hline 38 & PARTNERRELTD & BERMUDA & TOWER LIMITED & NEWZEALAND \\
\hline 39 & BANK OF BARODA & INDIA & GRUPO FINANCIERO BANORTES.A.B. DE C.V. & MEXICO \\
\hline 40 & HELIOS UNDERWRITING PLC & UNITED KINGDOM & CHANG HWA COMMERCIAL BANK LIMITED & TAIWAN \\
\hline 41 & FEDERATED NATIONAL HLDG CO & UNITED STATES & INDUSTRIAL BANK CO LTD & CHINA \\
\hline 42 & GRUPO FINANCIERO BANORTE S.A.B. DE C.V. & MEXICO & WUESTENROT \& WUERTTEMBERGISCHE AKTIENGES & GERMANY \\
\hline 43 & AXIS CAPITAL HOLDINGS LTD & BERMUDA & INTERLIFE GENERAL INSURANCE COMPANY SA & GREECE \\
\hline 44 & DONEGAL GROUPINC & UNITED STATES & HELIOS UNDERWRITING PLC & UNITED KINGDOM \\
\hline 45 & TOWER LIMITED & NEWZEALAND & WHITEMTNS INS GROUP LTD & BERMUDA \\
\hline 46 & DEUTSCHE POSTBANK AG & GERMANY & ALLIED IRISH BANKS PLC & IRELAND \\
\hline 47 & KANSAS CITY LIFE INS CO & UNITED STATES & NUERNBERGER BETELIGUNGS-AG & GERMANY \\
\hline 48 & ICICI BANK LIMITED & INDIA & GREAT EASTERN HOLDINGS LTD & SINGAPORE \\
\hline 49 & FIRST ACCEPTANCE CORP & UNITED STATES & CREDIT INDUSTRIE ET COMMERCIAL SA & FRANCE \\
\hline 50 & ASIA FINANCIAL HOLDINGS LTD. & HONG KONG & KINGSWAY FINANCIAL SVCS INC & CANADA \\
\hline
\end{tabular}

Source: Bloomberg LLP and author's calculations. 
Table 4e. Systemic vulnerability rankings, all firms: January 2001 - July 2016 (rankings based on a $\operatorname{VAR}(8)$ specification)

\begin{tabular}{|c|c|c|c|c|}
\hline \multicolumn{5}{|c|}{ Systemic vulnerability rankings } \\
\hline Ranking & Diebold-Yilmaz network & & Corrected Lanne-Nyberg-Diebold-Yilmaz network & \\
\hline 1 & INDEQUITY GROUP LIMITED & SOUTH AFRICA & INDEQUITY GROUP LIMITED & SOUTH AFRICA \\
\hline 2 & AXIS BANK LIMITED & INDIA & AXIS BANK LIMITED & INDIA \\
\hline 3 & BANK OF BARODA & INDIA & BANK OF BARODA & INDIA \\
\hline 4 & CHANG HWA COMMERCIAL BANK LIMITED & TAIWAN & CHANG HWA COMMERCIAL BANK LIMITED & TAIWAN \\
\hline 5 & HDFC BANK LIMITED & INDIA & TAIWAN LIFE INSURANCE COMPANY LIMITED & TAIWAN \\
\hline 6 & TAIWAN LIFE INSURANCE COMPANY LIMITED & TAIWAN & SHINKONG INSURANCE CO LTD & TAIWAN \\
\hline 7 & SHINKONG INSURANCE CO LTD & TAIWAN & HDFC BANK LIMITED & INDIA \\
\hline 8 & CENTRAL REINSURANCE CORPORATION & TAIWAN & STATE BANK OF INDIA & INDIA \\
\hline 9 & STATE BANK OF INDIA & INDIA & BANK OF INDIA & INDIA \\
\hline 10 & BANK OF INDIA & INDIA & CENTRAL REINSURANCE CORPORATION & TAIWAN \\
\hline 11 & ADITYA BIRLA NUVO LIMITED & INDIA & ADITYA BIRLA NUVO LIMITED & INDIA \\
\hline 12 & CHINA LIFE INSURANCE COMPANY LIMITED & TAIWAN & CHINA LIFE INSURANCE COMPANY LIMITED & TAIWAN \\
\hline 13 & ICICI BANK LIMITED & INDIA & ICICI BANK LIMITED & INDIA \\
\hline 14 & FIRST INSURANCE COMPANY LIMITED (THE) & TAIWAN & FIRST INSURANCE COMPANY LIMITED (THE) & TAIWAN \\
\hline 15 & TAIWAN FIRE \& MARINE INSURANCE COMPANY L & TAIWAN & TURNERS LTD & NEWZEALAND \\
\hline 16 & WURTTEMBERGISCHE LEBENSVERSICHERUNG AG & GERMANY & UNION INSURANCE COMPANY LIMITED & TAIWAN \\
\hline 17 & UNION INSURANCE COMPANY LIMITED & TAIWAN & WURTTEMBERGISCHE LEBENSVERSICHERUNG AG & GERMANY \\
\hline 18 & NUERNBERGER BETEILIGUNGS-AG & GERMANY & TAIWAN FIRE \& MARINE INSURANCE COMPANY L & TAIWAN \\
\hline 19 & PERSONAL GROUP HOLDINGS PLC & UNITED KINGDOM & GRUPO FINANCIERO BANORTES.A.B. DEC.V. & MEXICO \\
\hline 20 & TURNERS LTD & NEWZEALAND & SHANGHAI PUDONG DEVELOPMENT BANK & CHINA \\
\hline 21 & SINGAPORE RENSURANCE CORPORATION LIMITE & SINGAPORE & NISHI-NIPPON CITY BANK LTD & JAPAN \\
\hline 22 & VAUDOISE ASSURANCES HOLDING & SWTZERLAND & SBERBANK ROSSII OAO & RUSSIA \\
\hline 23 & SHANGHAI PUDONG DEVELOPMENT BANK & CHINA & NUERNBERGER BETELIGUNGS-AG & GERMANY \\
\hline 24 & GRUPO FINANCIERO BANORTES.A.B. DEC.V. & MEXICO & ASIA FINANCIAL HOLDINGS LTD. & HONG KONG \\
\hline 25 & NISHI-NIPPON CITY BANK LTD & JAPAN & FEDERATED NATIONAL HLDG CO & UNITED STATES \\
\hline 26 & UNITED OVERSEAS INSURANCE LIMITED & SINGAPORE & VAUDOISE ASSURANCES HOLDING & SWTZERLAND \\
\hline 27 & ASIA FINANCIAL HOLDINGS LTD. & HONG KONG & PERSONAL GROUP HOLDINGS PLC & UNITED KINGDOM \\
\hline 28 & INVESTEC LIMITED & SOUTH AFRICA & SINGAPORE RENSURANCE CORPORATION LIMITE & SINGAPORE \\
\hline 29 & GREAT EASTERN HOLDINGS LTD & SINGAPORE & PANIN FINANCIAL TBK PT & INDONESIA \\
\hline 30 & SHIZUOKA BANK LTD (THE) & JAPAN & TOWER LIMITED & NEWZEALAND \\
\hline 31 & BANK OF YOKOHAMA LTD & JAPAN & AVIVA SIGORTA AS & TURKEY \\
\hline 32 & HIROSHIMA BANK LIMITED (THE) & JAPAN & HUNTINGTON BANCSHARES & UNITED STATES \\
\hline 33 & UNIQA INSURANCE GROUPAG & AUSTRIA & PANINVEST TBK PT & INDONESIA \\
\hline 34 & TOWER LIMITED & NEWZEALAND & PING AN BANK COMPANY LIMITED & CHINA \\
\hline 35 & MARKEl CORP & UNITED STATES & KINGSWAY FINANCIAL SVCS INC & CANADA \\
\hline 36 & HUNTINGTON BANCSHARES & UNITED STATES & INVESTEC LIMITED & SOUTH AFRICA \\
\hline 37 & KANSAS CITY LIFE INS CO & UNITED STATES & HAITONG SECURITIES CO. LIMITED & CHINA \\
\hline 38 & FAIRFAX FINANCIAL HOLDINGS & CANADA & CHINA TAIPING INSURANCE HOLDINGS COMPANY LIMITED & HONG KONG \\
\hline 39 & FBD HOLDINGS PLC & IREAND & FAIRFAX FINANCIAL HOLDINGS & CANADA \\
\hline 40 & ANADOLU ANONIM TURK SIGORTA SIRKETI & TURKEY & FBD HOLDINGS PLC & IRELAND \\
\hline 41 & VITTORIA ASSICURAZIONI SPA & ITALY & TORONTO DOMINION BANK & CANADA \\
\hline 42 & WUESTENROT \& WUERTTEMBERGISCHE AKTIENGES & GERMANY & PRUDENTIAL PLC & UNITED KINGDOM \\
\hline 43 & 77 BANK LIMITED (THE) & JAPAN & BNP PARIBAS SA & FRANCE \\
\hline 44 & E-L FINANCIAL CORP LTD & CANADA & POWER FINANCIAL CORP & CANADA \\
\hline 45 & PING AN BANK COMPANY LIMITED & CHINA & NORDEA BANK AB & SWEDEN \\
\hline 46 & CHIBA BANK LTD (THE) & JAPAN & AVIVA PLC & UNITED KINGDOM \\
\hline 47 & RLI CORP & UNITED STATES & POWER CORP CANADA & CANADA \\
\hline 48 & CHINA MINSHENG BANKING CORPORATION LIMITED & CHINA & KEMPER CORP/ DE & UNITED STATES \\
\hline 49 & HANG SENG BANK LIMITED & HONG KONG & GOLDMAN SACHS GROUPINC & UNITED STATES \\
\hline 50 & ERIE INDEMNITY CO -CL A & UNITED STATES & U S BANCORP & UNITED STATES \\
\hline
\end{tabular}

Source: Bloomberg LLP and author's calculations. 
Only a handful of institutions are simultaneously in the top risk and vulnerability rankings. In the case of the DY rankings, it is Sberbank, a Russian bank, during the 2005 - 2008, and CPP Group, a British insurer, during 2013 - 2016. In the case of the CLNDY rankings, it is only Banca Popolare, an Italian bank, during 2001 - 2004. A glance at Tables 3 and 4 reveals that the headquarters of the riskiest firms are mainly in advanced economies. U.S. firms dominate the top rankings during 2005-2008, coinciding with the subprime crisis in the United States, and European firms dominate during 2009 - 2012, coinciding with the sovereign debt crisis.

Table 5 provides further confirmation, with firms in emerging economies losing systemic importance during 2005 - 2012 in the DY rankings. The CLNDY ranking, however, suggests that these firms are not systemic at all. In regards to vulnerability, emerging market firms appear over-represented relative to their share in the sample. It is worth highlighting that in advanced economies, the risky firms tend to be in North America and Europe, and the vulnerable firms in Asia and Oceania.

Table 5. Top fifty systemic firms, by headquarter location (ranking based on a VAR (8) specification)

\begin{tabular}{|c|c|c|c|c|c|}
\hline & \multicolumn{5}{|c|}{ Period } \\
\hline & 2001 to 2004 & 2005 to 2008 & $2009-2012$ & $2013-2016$ & $2001-2016$ \\
\hline \multicolumn{6}{|c|}{ Panel A: Systemic risk ranking } \\
\hline \multicolumn{6}{|c|}{ Diebold-Yilmaz } \\
\hline Advanced economies & 36 & 41 & 50 & 33 & 49 \\
\hline Emerging economies & 14 & 9 & 0 & 17 & 1 \\
\hline \multicolumn{6}{|c|}{ Corrected Lanne-Nyberg-Diebold-Yilmaz } \\
\hline Advanced economies & 50 & 50 & 50 & 48 & 50 \\
\hline Emerging economies & 0 & 0 & 0 & 2 & 0 \\
\hline \multicolumn{6}{|c|}{ Panel B: Systemic vulnerability ranking } \\
\hline \multicolumn{6}{|c|}{ Diebold-Yilmaz } \\
\hline Advanced economies & 38 & 33 & 34 & 33 & 32 \\
\hline Emerging economies & 12 & 17 & 6 & 7 & 18 \\
\hline \multicolumn{6}{|c|}{ Corrected Lanne-Nyberg-Diebold-Yilmaz } \\
\hline Advanced economies & 29 & 29 & 33 & 35 & 32 \\
\hline Emerging economies & 21 & 21 & 7 & 5 & 18 \\
\hline
\end{tabular}

Source: Bloomberg LLP and author's calculations.

While the DY and CLNDY rankings can be strikingly different at the individual firm level, they convey roughly the same information on how the probability distribution of rankings evolves over time, as Figure 3 and 4 indicate. Figure 3 shows the distribution of systemic risk rankings for different categories of firms, with the bank category including not only banks but also 
securities and broker dealers. During the pre-crisis period of 2001 to 2004, the distribution of systemic risk rankings in the banking sector was highly skewed towards the top one hundred rankings, especially visible in the inverted pear-shaped CLNDY distribution (bottom panel, Figure 3a). During the crisis period, 2005 to 2008, the rankings of the banks were roughly uniformly distributed but still slightly concentrated in the upper range of values. At the time of the European sovereign debt crisis, 2009 to 2012, bank ranking skewed towards the upper end, and they have remained highly skewed during the secular stagnation period, 20013 to 2016.

Figure 3a. Banks: systemic risk rankings, probability distribution evolution (ranking based on a VAR(8) specification)
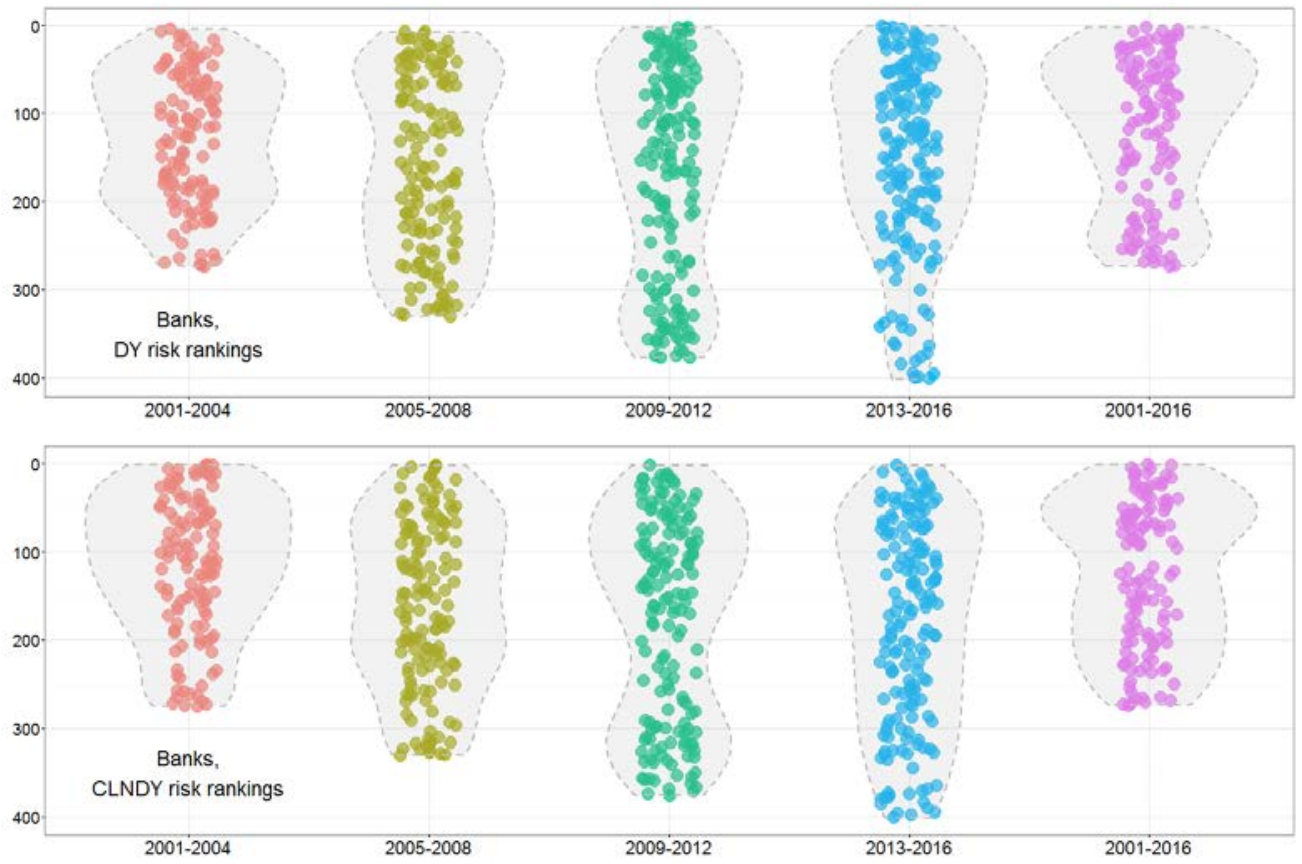

Rankings in reverse order, right axis. A lower ranking corresponds to higher risk Source: Bloomberg LLP and author's calculations.

The distributions of risk rankings of life insurance and property/casualty/health insurance firms have evolved quite differently. In the earlier part of the sample, quite a large proportion of large insurers rank among the top hundred firms (Figure 3b). The situation reversed during the crisis period but the industry risk rankings rose during the 2009 - 2012 period. More recently, the distribution of risk rankings in the industry appears uniform.

Systemic risk in the property/casualty/health insurance sector has never been high despite an upward migration in the crisis period of 2005 - 2008 (Figure 3c). Finally, if the whole sample period of 2001 - 2016 is considered, the emerging picture is one that places banks at the top of the risk rankings, followed in descending order by life insurers and property/casualty/health insurers. This is consistent with conclusions reached by the International Monetary Fund (IMF, 2016) using quite a different set of analytical methods. 
Figure 3b. Life insurers: systemic risk rankings, probability distribution evolution (ranking based on a VAR(8) specification)
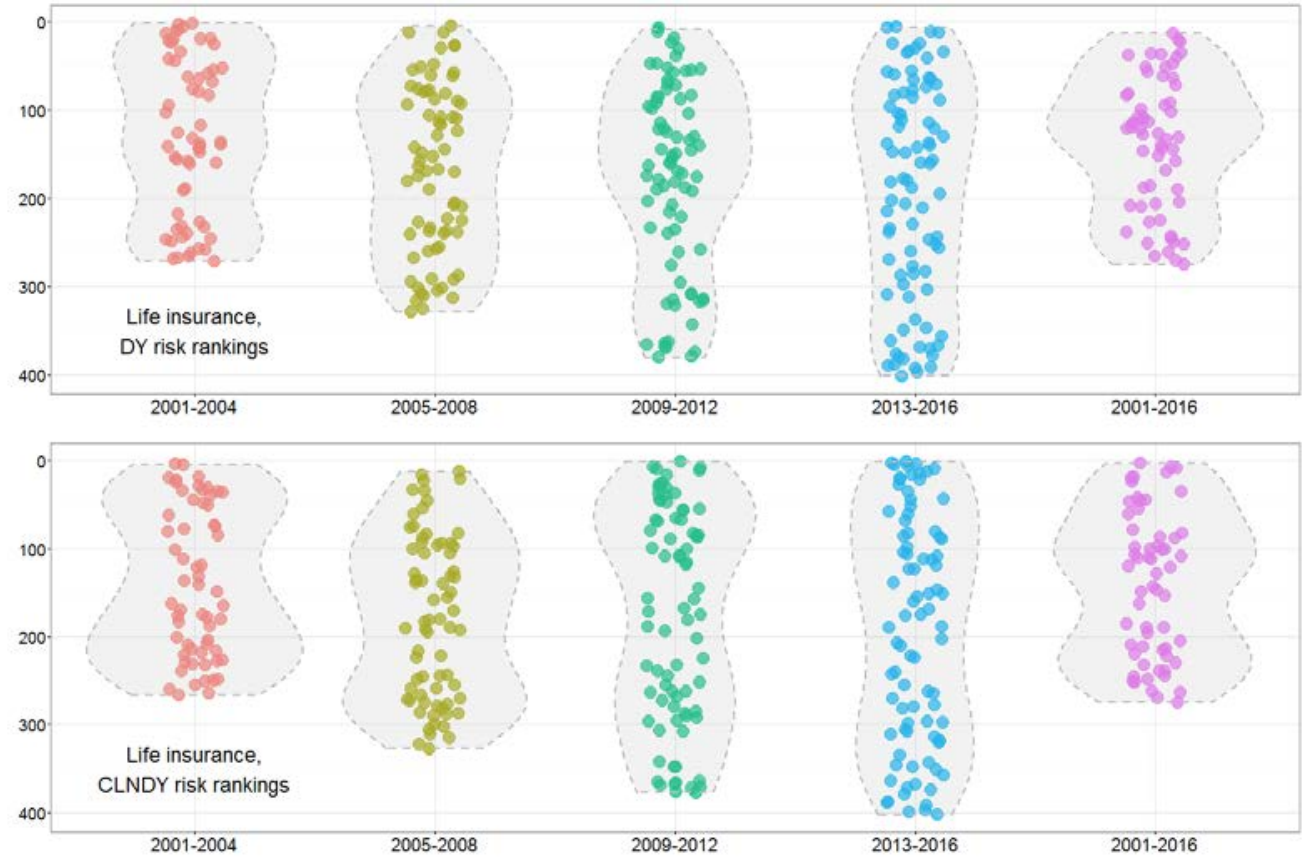

Rankings in reverse order, right axis. A lower ranking corresponds to higher risk Source: Bloomberg LLP and author's calculations.

Figure 3c. Property/casualty/health insurers: systemic risk rankings, probability distribution evolution (ranking based on a VAR(8) specification)
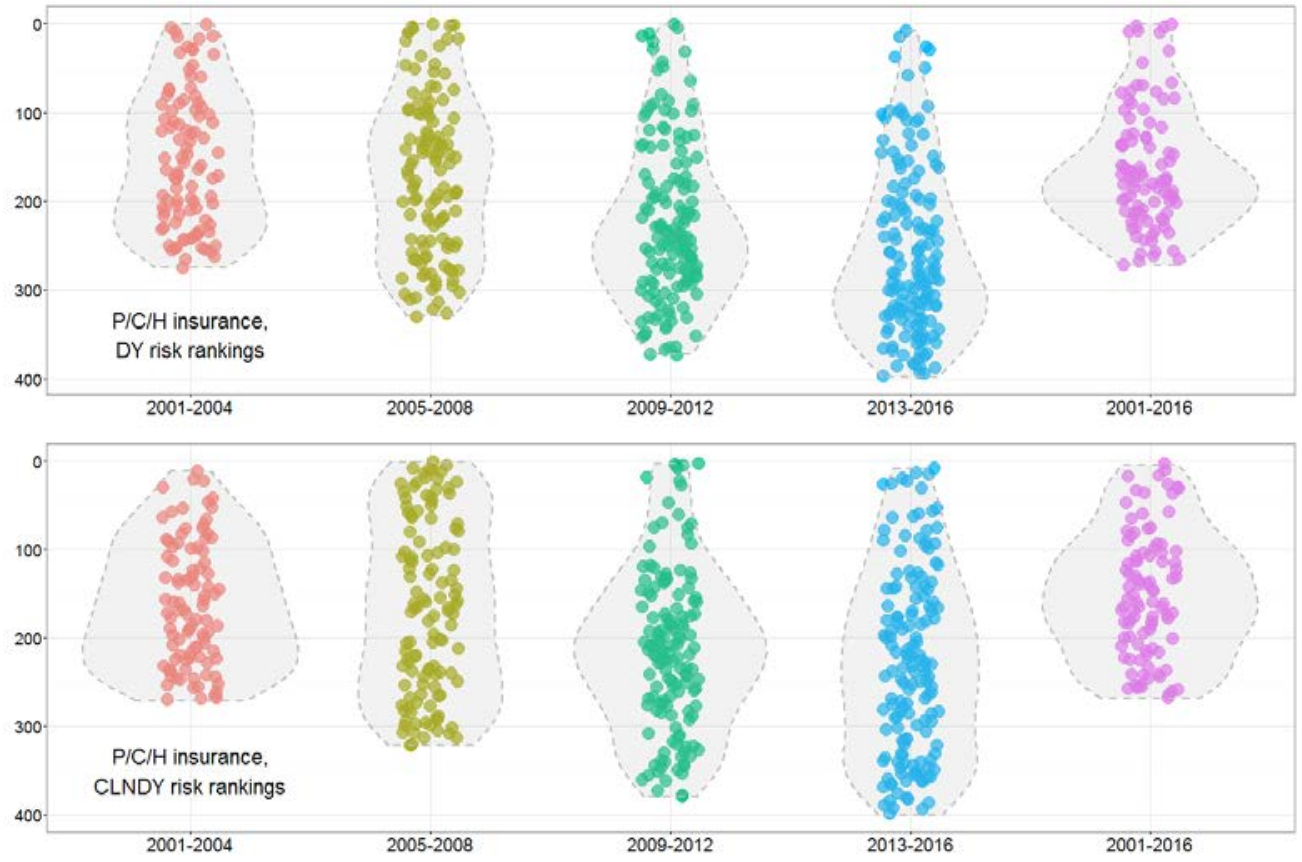

Rankings in reverse order, right axis. A lower ranking corresponds to higher risk Source: Bloomberg LLP and author's calculations. 
The analysis of vulnerability distributions in the banking sector is a mirror image of its risk distributions (Figure 4a). In the earlier part of the sample, most banks ranked in the bottom two thirds of the distribution and have remained there since then despite a slight upward shift during $2009-2012$.

Figure 4a. Banks: systemic vulnerability rankings, probability distribution evolution (ranking based on a VAR(8) specification, right axis)

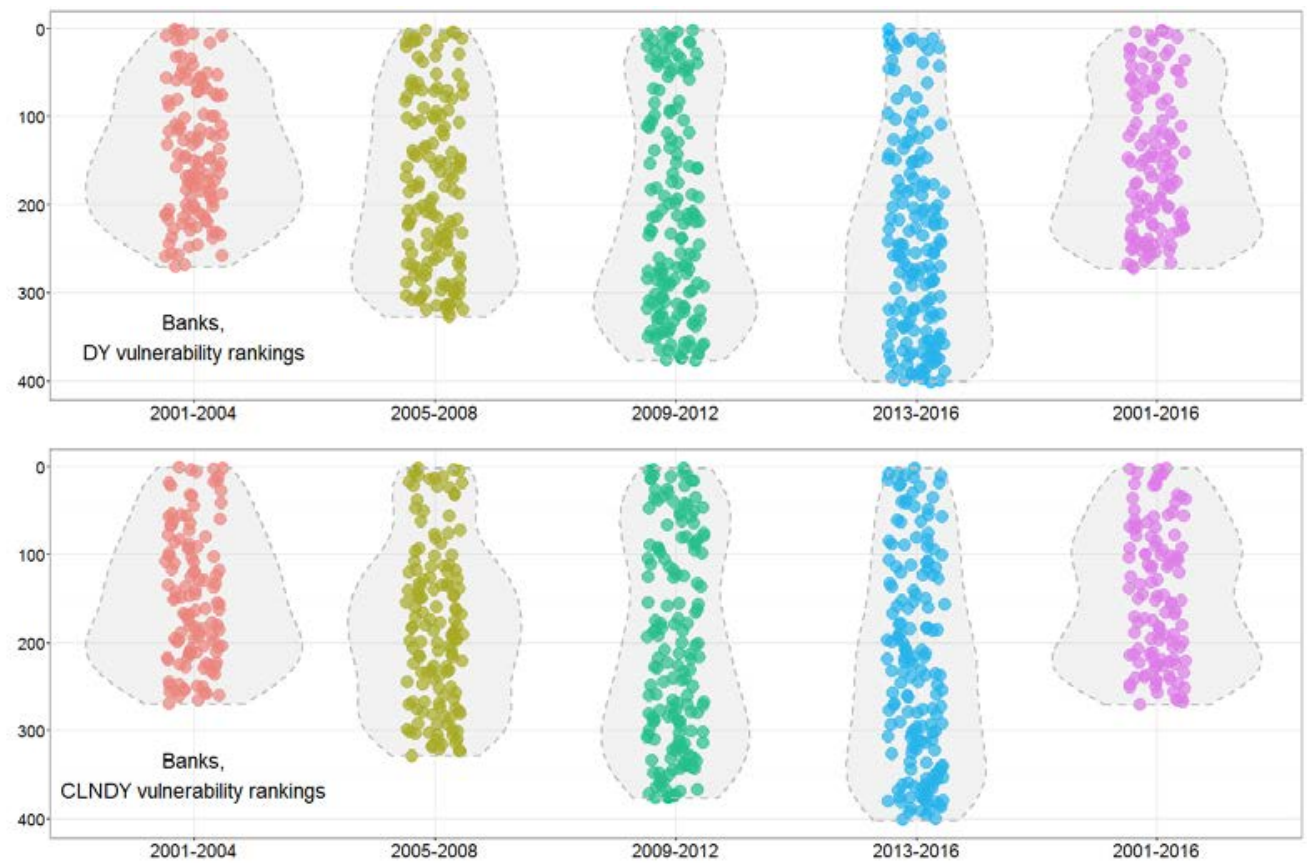

Rankings in reverse order, right axis. A lower ranking corresponds to higher vulnerability Source: Bloomberg LLP and author's calculations.

In contrast, the vulnerability rankings of life insurance firms were concentrated in the upper region up to the 2008 global financial crisis, but they have declined since then.. The current distribution in 2013-2016, however, is bimodal with a peak in the top quarter of the distribution, and the other peak in the bottom quarter (Figure 4b). While property/casualty/health insurance firms have not ranked among the riskiest firms in the system, the opposite is true regarding their vulnerability, with many firms in the sector concentrated in the top rankings, regardless of whether the DY or CLNDY ranking is used, during 2001 - 2008. Although the concentration of vulnerability rankings has become wider since 2008, property/casualty/health insurance firms remain more vulnerable vis-a-vis banks and the life insurance firms. The full sample analysis also supports this fact. 
Figure 4b. Life insurers: systemic vulnerability rankings, probability distribution evolution (ranking based on a VAR(8) specification, right axis)
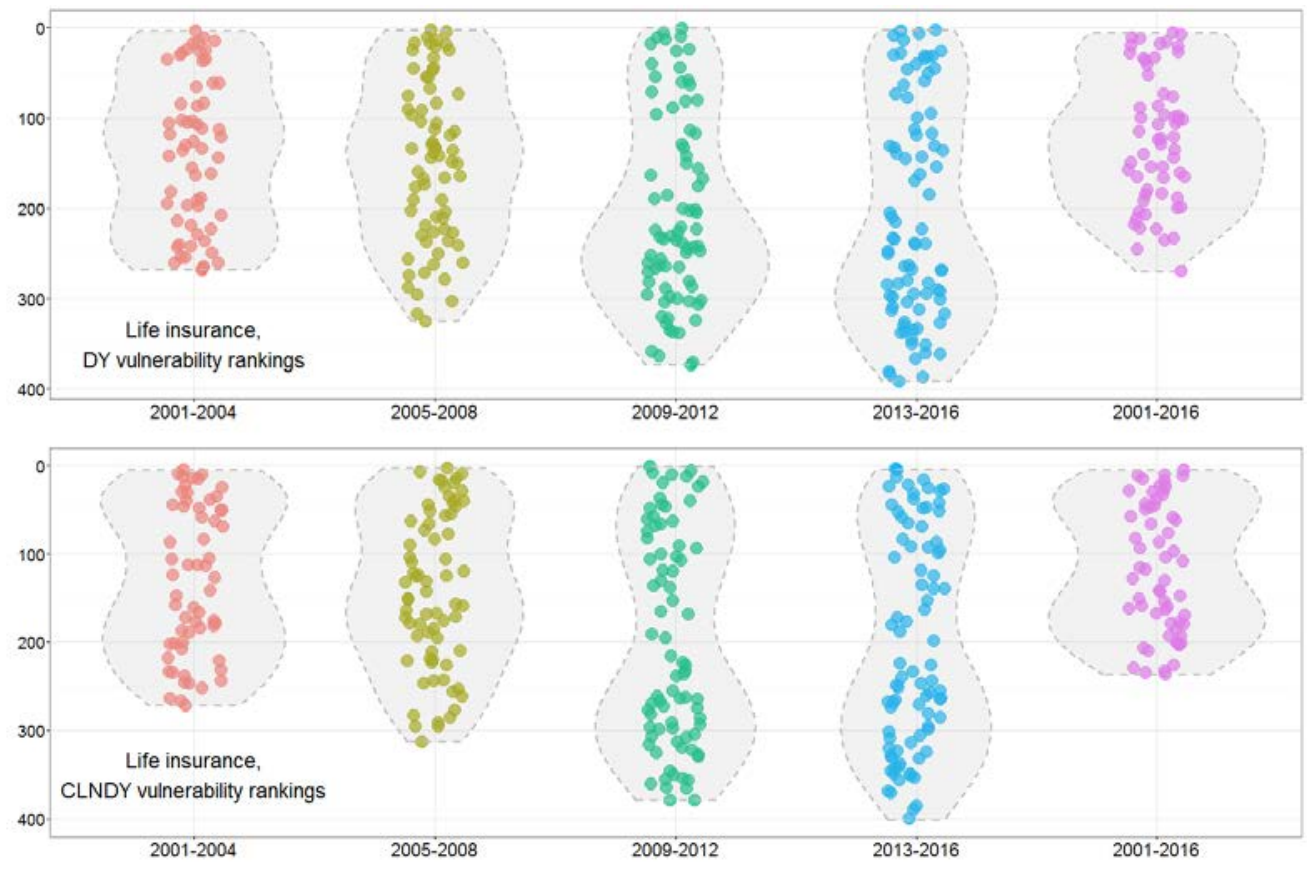

Rankings in reverse order, right axis. A lower ranking corresponds to higher vulnerability Source: Bloomberg LLP and author's calculations.

Figure 4c. Property/casualty/health insurers: systemic vulnerability rankings, probability distribution evolution (ranking based on a VAR(8) specification, right axis)

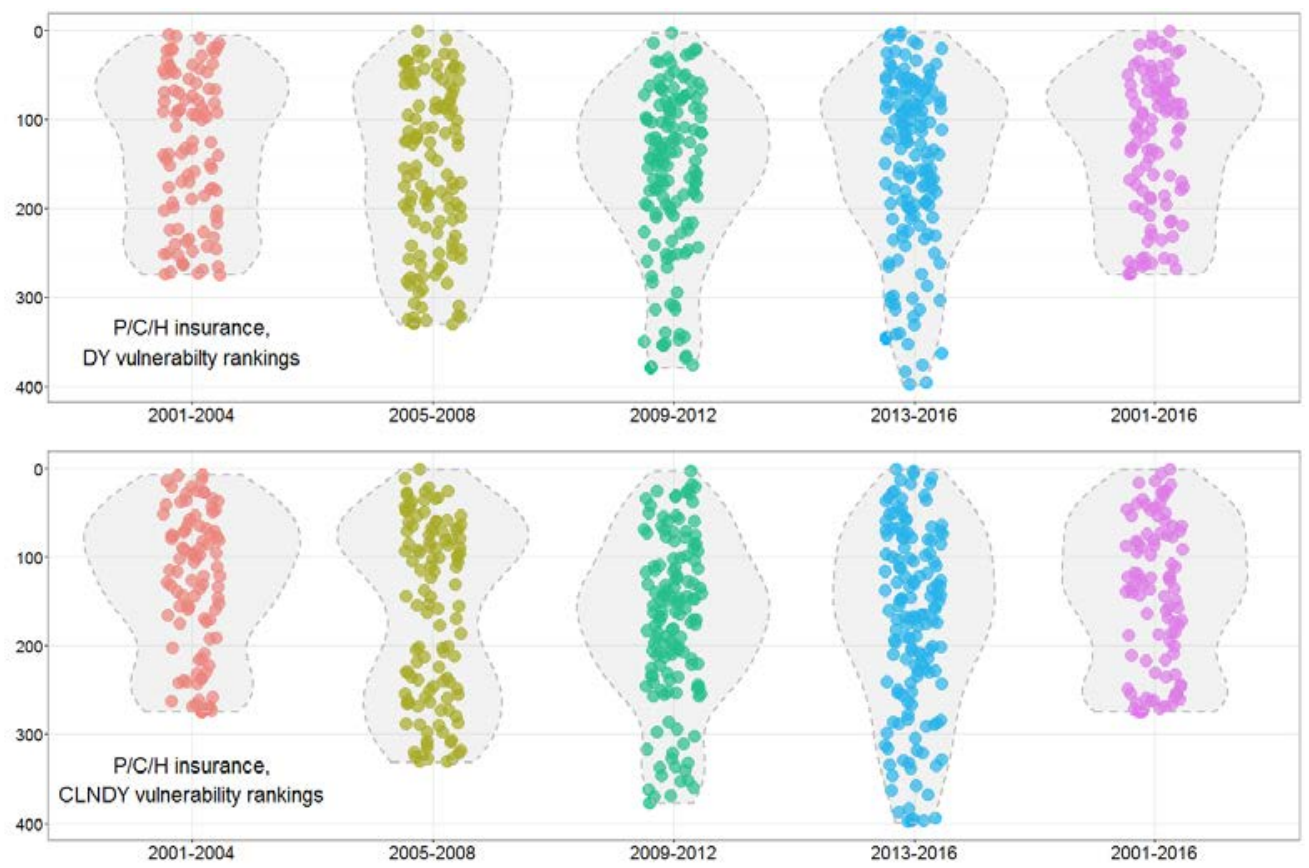

Rankings in reverse order, right axis. A lower ranking corresponds to higher vulnerability Source: Bloomberg LLP and author's calculations. 
To complement the analysis above, Table 6 shows the industry share in the top fifty systemic firms. In general, the DY rankings weight bank, which represent more than sixty percent of the riskiest firms since 2009, more heavily than the CLNDY rankings. In both rankings, banks are the riskiest, followed by life insurance firms, and then property/casualty/health insurance firms. There was a reversal, however, during the crisis period in 2005 - 2008, when the importance of the property/casualty/health insurance sector increased. The share of vulnerabilities is more evenly distributed in the CLNDY rankings than in the DY rankings.

Table 6. Top fifty systemic firms, by industry, in percent (ranking based on a VAR (8) specification)

\begin{tabular}{|c|c|c|c|}
\hline Banks & $\begin{array}{c}\text { Life } \\
\text { insurance }\end{array}$ & $\begin{array}{c}\text { Insurance: } \\
\text { Property and } \\
\text { Casualty; Health }\end{array}$ & $\begin{array}{c}\text { Security } \\
\text { brokers and } \\
\text { dealers }\end{array}$ \\
\hline
\end{tabular}

Diebold-Yilmaz, risk rankings

$\begin{array}{lllll}2001-2004 & 44 & 30 & 24 & 2 \\ 2005-2008 & 52 & 14 & 28 & 6 \\ 2009-2012 & 66 & 16 & 18 & 0 \\ 2013-2016 & 62 & 22 & 12 & 4 \\ 2001-2016 & 64 & 18 & 16 & 2\end{array}$

Corrected Lanne-Nyberg-Diebold-Yilmaz, risk rankings

$\begin{array}{lllll}2001-2004 & 58 & 30 & 12 & 0 \\ 2005-2008 & 40 & 14 & 42 & 4 \\ 2009-2012 & 50 & 32 & 18 & 0 \\ 2013-2016 & 46 & 32 & 16 & 6 \\ 2001-2016 & 50 & 24 & 22 & 4\end{array}$

Diebold-Yilmaz, vulnerability rankings

$\begin{array}{lllll}2001-2004 & 28 & 26 & 44 & 2 \\ 2005-2008 & 30 & 28 & 40 & 2 \\ 2009-2012 & 58 & 20 & 22 & 0 \\ 2013-2016 & 34 & 32 & 34 & 0 \\ 2001-2016 & 38 & 28 & 32 & 2\end{array}$

Corrected Lanne-Nyberg-Diebold-Yilmaz, vulnerability rankings

$\begin{array}{lllll}2001-2004 & 28 & 34 & 36 & 2 \\ 2005-2008 & 32 & 30 & 34 & 4 \\ 2009-2012 & 48 & 26 & 26 & 0 \\ 2013-2016 & 36 & 30 & 34 & 0 \\ 2001-2016 & 30 & 34 & 32 & 4\end{array}$

Source: Bloomberg LLP and author's calculations. 


\section{Conclusions}

Analyzing systemic risk in financial networks has become increasingly important in the aftermath of the financial crisis. To complement network analysis based on bilateral exposures, academics and policy practitioners have developed techniques for constructing financial networks based on the comovement of securities prices. In particular, Diebold and Yilmaz (2015) propose using variance decomposition networks, which can capture the directionality of spillovers between firms.

One disadvantage in Diebold and Yilmaz original methodology, however, is its reliance on Pesaran-Shin (1998) GFEVDs, which do not add to unity. This makes difficult to compare the risk contribution and ranking of a firm at two different points in time. It is possible, however, to enhance the Diebold-Yilmaz methodology while preserving its intuition by simply replacing the the Lanne-Nyberg (2016) decomposition, which adds to unity, for the Pesaran-Shin decomposition.

Different variance decompositions, however, could produce substantially different systemic risk rankings. In a global financial network comprising a large number of financial firms in advanced and emerging market economies, the DY and CLNDY systemic risk rankings differed markedly. When comparing the top fifty firms, less than half of the firms were in both rankings. The choice decomposition method, however, did not affect systemic vulnerability rankings to the same extent. While the rankings of individual firms may be different, the evolution of the distribution of rankings over time was quite similar using either of the two methods.

This paper favors the use of the CLNDY rather than the original DY network for assessing the systemic risk contribution of firms, and assessing their systemic importance in the financial system. From an anecdotal perspective, the CLNDY risk ranking appears to do a better job than the DY risk ranking in selecting the top riskiest firms in the 2005- 2008 and the $2013-2016$ periods. Survivorship bias, however, may have influenced this result since the analysis did not include delisted firms such as Lehman Brothers and Merrill Lynch that had a major role during the 2008 crisis. Regardless of this paper preference, however, the differences induced by the choice of decomposition suggest caution when using systemic risk rankings for guiding financial regulation and policy recommendations. 


\section{References}

Anand, K., G. Bedard-Page, and V. Traclet, 2014, "Stress Testing the Canadian Banking System: A System-Wide Approach,” Financial System Review, Bank of Canada, pp. 61- 68.

Anand, K., B. Craig, and G. von Peter, 2015, "Filling in the Blanks: Network Structure and Interbank Contagion,” Quantitative Finance, Vol. 15, No. 4, pp. 625 - 636.

Baba, K., R. Shibata, and M. Sibuya, 2004, "Partial Correlation and Conditional Correlation as Measures of Conditional Independence,” Australian \& New Zealand Journal of Statistics, Vol. 46, pp. 657-664.

Banco de Mexico, 2015, Reporte sobre el Sistema Financiero, November.

Battiston, S., M. Puliga, R. Kaushik, P. Tasca, and G. Caldarelli, 2012,’DebtRank: Too Central to Fail? Financial Networks, the FED and Systemic Risk,” Scientific Reports, Vol. 2, Article 541.

Billio, M., M. Getmansky, A. Lo, and L. Pelizzon, 2012, “Econometric Measures of Connectedness and Systemic Risk in the Finance and Insurance Sectors,” Journal of Financial Economics, Vol. 104, pp. 535 - 559.

Bookstaber, R., and M. Paddrik, 2015, “An Agent-Based Model for Crisis Liquidity Dynamics,” OFR Working Paper 15 - 18, Office of Financial Research, U.S. Department of the Treasury.

Brunnermeier, M.K., and L.H. Pedersen, 2009, “Market Liquidity and Funding Liquidity,” Review of Financial Studies, Vol. 22, No. 6, pp. 2201-2238.

Chan-Lau, J.A., 2015, “ABBA: An Agent-Based Model of the Banking System,” mimeo. Available at papers.ssrn.com/sol3/Delivery.cfm?abstractid=2784228.

Chan-Lau, J.A., C. Chuang, J.C. Duan, and W. Sun, 2016, "Banking Network and Systemic Risk via Forward-Looking Partial Default Correlation,” mimeo, International Monetary Fund and Credit Research Initiative at the Risk Management Institute, National University of Singapore.

Davis, R., P. Zang, T. Zheng, 2015, “Sparse Vector Autoregressive Modeling,” forthcoming in Journal of Computational and Graphical Stastistics.

D’Errico, M., S. Battiston, and S. Gurciullo, 2016, "DebtRank and the Network of Leverage,” Journal of Alternative Investments, Vol. 18, No. 4.

Demekas, D., J.A. Chan-Lau, C. Caceres, F. Ohnsorge, N. Rendak , K. Tintchev, and K. Youssef, 2013, "Mandatory Financial Stability Assessments Under the Financial Sector Assessment Program: Update,” International Monetary Fund, Washington, D.C. 
Demirer, M., F.X. Diebold, L. Liu, and K. Yilmaz, 2015, “Estimating Global Bank Network Connectedness,” Koc University - TUSIAD Economic ResearchForum, Working Paper No. 1512.

Derenyi, I., G. Palla, and T. Vicsek, 2005, “Clique Percolation in Random Networks,” Physical Review Letters, Vol. 94, 160202.

Diebold, F.X., and K. Yilmaz, 2014, “On the Network Topology of Variance Decompositions: Measuring the Connectedness of Financial Firms,” Journal of Econometrics, Vol. 182, No. 1, pp. 119 - 134.

Easley, D., and J. Kleinberg, 2010, Networks, Crowds, and Markets: Reasoning About a Highly Connected World, Cambridge University Press.

Eisenberg, L., and T.H. Noe, 2001, “Systemic Risk in Financial Systems,” Management Science, Vol. 47, No. 2, pp. 236-249.

Elliott, M., B. Golub, and M.O. Jackson, 2014, “Financial Networks and Contagion,” American Economic Review, Vol. 104, No. 10, pp. 3115-53.

Fan, J., J. Lv, and L. Qi, 2011, “Sparse High-Dimensional Models in Economics,” Annual Review of Economics, Vol. 3, pp. 291 - 317.

Financial Stability Board, 2009, Guidance to Assess the Systemic Importance of Financial Institutions (Basel).

Gai, P., and S. Kapadia, 2010, “Contagion in Financial Networks,” Proceedings of the Royal Society A, Vol. 466, No. 2120, pp. 2401-23.

Hamilton, J., 1994, Time Series Analysis, Princeton University Press.

International Monetary Fund, 2016, “The Insurance Sector: Trends and Systemic Risk Implications,” chapter 3 in Global Financial Stability Report, Washington, D.C.

Jackson, M.O., 2010, Social and Economic Networks, Princeton University Press.

James, G., D. Witten, T. Hastie, and R. Tibshirani, 2013, An Introduction to Statistical Learning - with Applications in $R$ (Springer Verlag).

Kenett, D.Y., M. Tumminello, A. Madi, G. Gur-Gershgoren, R.N. Mantegna, and E. Ben-Jacob, 2010, "Dominating Clasp of the Financial Sector Revealed by Partial Correlation Analysis of the Stock Market,” PLoS ONE Vol. 5, No. 12: e15032.

Kenett, D.Y., X. Huang, I. Vodenska, S. Havlin, and H.E. Stanley, 2014, "Partial Correlation Analysis: Applications for Financial Markets,” forthcoming in Quantitative Finance. 
Khare, K., S.-Y. Oh, and B. Rajaratnam, 2015, “A Convex Pseudo-Likelihood Framework for High Dimensional Partial Correlation Estimation with Convergence Guarantees,” Journal of the Royal Statistical Society, Series B (Statistical Methodoology), pp. 803 - 825.

Koop, G., 2013, “Forecasting with Medium and Large Bayesian VARs,” Journal of Applied Econometrics, Vol. 28, pp. 177 - 203.

Koop, G., H. H. Pesaran, and S. Potter, 1996, “Impulse Response Analysis in Nonlinear Multivariate Models,” Journal of Econometrics, Vol. 74, pp. 119-147.

Lanne, M., and H. Nyberg, 2016, “Generalized Forecast Error Variance Decomposition for Linear and Nonlinear Multivariate Models,” Oxford Bulletin of Economics and Statistics, Vol. 78, No. 4, pp. 595-603.

Li, J., and W. Chen, 2014, “Forecasting Macroeconomic Time Series: Lasso-based Approaches and their Forecast Combinations with Dynamic Factor Models," International Journal of Forecasting, Vol. 30, No. 4, pp. 996 - 1015.

Mantegna, R.N., 1999, “Hierarchical Structure in Financial Markets,” The European Physical Journal B, Vol. 11, pp. 193 - 197.

Montagna, M., and C. Kok, 2013, "Multi-layered Interbank Model for Assessing Systemic Risk,” Kiel Working Papers No. 1873, Kiel Institute for the World Economy.

Nicholson, W., D. Matteson, and J. Bien, 2016, "VARX-L: Structured Regularization for Large Vector Autoregressions with Exogenous Variables,” mimeo, Cornell University.

Oh, S-Y., O. Dalal, K. Khare, and B. Rajaratnam, 2014, “Optimization Methods for Sparse Pseudo-Likelikhood Graphical Model Selection,” Advances in Neural Information Processing Systems, pp. 667 - 675.

Opgen-Rhein, R., and K. Strimmer, 2007, “Accurate Ranking of Differentially Expressed Genes by a Distribution-Free Shrinkage," Statistical Applications in Genetics and Molecular Biology, Vol. 6, No. 1 (available online).

Pesaran, H.H., and Y. Shin, 1998, "Generalized Impulse Response Analysis in Linear Multivariate Models,” Economic Letters, Vol. 58, No. 1, pp. 17 - 29.

Schafer, J., and K. Strimmer, 2005, “A Shrinkage Approach to Large-Scale Covariance Matrix Estimation and Implications for Functional Genomics,” Statistical Applications in Genetics and Molecular Biology, Vol. 4, No 1 (available online).

Song, S., and P.J. Bickel, 2011, “Large Vector Auto Regressions,” mimeo, University of California, Berkeley.

Tibshirani, R., 1995, "Regression Shrinkage and Selection via the Lasso,” Journal of the Royal Statistical Society, Series B, Vol. 58, No. 1, pp. 267-288. 
Upper, C., 2011, “Simulation Methods to Assess the Danger of Contagion in Interbank Markets," Journal of Financial Stability, Vol. 3, No. 7, pp. 111-125.

Zou, H., and T. Hastie, 2005, "Regularization and Variable Selection via the Elastic Net," Journal of the Royal Statistical Society, Series B, Vol. 67, pp. $301-320$.

Zou, H., and H. Zhang, 2009, “On the Adaptive Elastic Net with a Diverging Number of Parameters,” Annals of Statistics, Vol. 37, 1733. 


\section{Appendix. Selected Tables, January 2001 - July 2016}

Table A1. Advanced Asia - systemic risk ranking (ranking based on a VAR (8) specification)

\begin{tabular}{|c|c|c|c|}
\hline Ranking & Firm & Country & Industry \\
\hline \multicolumn{4}{|c|}{ DY rankings } \\
\hline 1 & Macquarie Group Limited & Australia & Security Brokers \& Dealers \\
\hline 2 & Dai-Ichi Life Insurance Company, Ltd (Th & Japan & Insurance - Life \\
\hline 3 & China Taiping Insurance Holdings Company Limited & Hong Kong & Insurance - Life \\
\hline 4 & Australia And New Zealand Banking Group & Australia & Banks And S\&Ls \\
\hline 5 & Nomura Holdings Incorporated & Japan & Security Brokers \& Dealers \\
\hline 6 & T\&D Holdings Incorporated & Japan & Insurance - Life \\
\hline 7 & National Australia Bank Limited & Australia & Banks And S\&Ls \\
\hline 8 & Bank Of Kyoto Ltd & Japan & Banks And S\&Ls \\
\hline 9 & Sony Financial Holdings Inc. & Japan & Insurance - Life \\
\hline 10 & Qbe Insurance Group Limited & Australia & Insurance - Prop/Cas/Health \\
\hline 11 & Anicom Holdings Incorporation & Japan & Insurance - Prop/Cas/Health \\
\hline 12 & Westpac Banking Corporation & Australia & Banks And S\&Ls \\
\hline 13 & Dream Incubator Incorporated & Japan & Insurance - Prop/Cas/Health \\
\hline 14 & Fukuoka Financial Group, Inc & Japan & Banks And S\&Ls \\
\hline 15 & Shinsei Bank Limited & Japan & Banks And S\&Ls \\
\hline 16 & Shizuoka Bank Ltd (The) & Japan & Banks And S\&Ls \\
\hline 17 & Sumitomo Mitsui Financial Group Inc & Japan & Banks And S\&Ls \\
\hline 18 & Challenger Financial Services Group Ltd & Australia & Insurance - Life \\
\hline 19 & Commonwealth Bank Of Australia & Australia & Banks And S\&Ls \\
\hline 20 & Mitsubishi Ufj Financial Group Incorpora & Japan & Banks And S\&Ls \\
\hline 21 & Chiba Bank Ltd (The) & Japan & Banks And S\&Ls \\
\hline 22 & Nishi-Nippon City Bank Ltd & Japan & Banks And S\&Ls \\
\hline 23 & Hokuhoku Financial Group Incorporated & Japan & Banks And S\&Ls \\
\hline 24 & Sompo Japan Nipponkoa Holdings Inc & Japan & Insurance - Prop/Cas/Health \\
\hline 25 & Chugoku Bank Limited (The) & Japan & Banks And S\&Ls \\
\hline 26 & Hiroshima Bank Limited (The) & Japan & Banks And S\&Ls \\
\hline 27 & Hachijuni Bank Limited (The) & Japan & Banks And S\&Ls \\
\hline 28 & 77 Bank Limited (The) & Japan & Banks And S\&Ls \\
\hline 29 & Gunma Bank Ltd & Japan & Banks And S\&Ls \\
\hline 30 & Joyo Bank Limited (The) & Japan & Banks And S\&Ls \\
\hline
\end{tabular}

\begin{tabular}{|c|c|c|c|}
\hline Ranking & Firm & Country & Industry \\
\hline \multicolumn{4}{|c|}{ CLNDY rankings } \\
\hline 1 & Australia And New Zealand Banking Group & Australia & Banks And S\&Ls \\
\hline 2 & Macquarie Group Limited & Australia & Security Brokers \& Dealers \\
\hline 3 & Commonwealth Bank Of Australia & Australia & Banks And S\&Ls \\
\hline 4 & National Australia Bank Limited & Australia & Banks And S\&Ls \\
\hline 5 & Westpac Banking Corporation & Australia & Banks And S\&Ls \\
\hline 6 & Oversea-Chinese Banking Corporation Limi & Singapore & Banks And S\&Ls \\
\hline 7 & United Overseas Bank Limited & Singapore & Banks And S\&Ls \\
\hline 8 & Dbs Group Holdings Ltd & Singapore & Banks And S\&Ls \\
\hline 9 & Bank Of East Asia Limited (The) & Hong Kong & Banks And S\&Ls \\
\hline 10 & Suncorp Group Limited & Australia & Insurance - Prop/Cas/Health \\
\hline 11 & Shizuoka Bank Ltd (The) & Japan & Banks And S\&Ls \\
\hline 12 & Mitsubishi Ufj Financial Group Incorpora & Japan & Banks And S\&Ls \\
\hline 13 & Aia Group Limited & Hong Kong & Insurance - Life \\
\hline 14 & Hang Seng Bank Limited & Hong Kong & Banks And S\&Ls \\
\hline 15 & Dai-Ichi Life Insurance Company, Ltd (Th & Japan & Insurance - Life \\
\hline 16 & Challenger Financial Services Group Ltd & Australia & Insurance - Life \\
\hline 17 & Sumitomo Mitsui Financial Group Inc & Japan & Banks And S\&Ls \\
\hline 18 & Boc Hong Kong (Holdings) Ltd & Hong Kong & Banks And S\&Ls \\
\hline 19 & Nomura Holdings Incorporated & Japan & Security Brokers \& Dealers \\
\hline 20 & Insurance Australia Group Limited & Australia & Insurance - Prop/Cas/Health \\
\hline 21 & Sony Financial Holdings Inc. & Japan & Insurance - Life \\
\hline 22 & Bank Of Kyoto Ltd & Japan & Banks And S\&Ls \\
\hline 23 & T\&D Holdings Incorporated & Japan & Insurance - Life \\
\hline 24 & Chiba Bank Ltd (The) & Japan & Banks And S\&Ls \\
\hline 25 & Hokuhoku Financial Group Incorporated & Japan & Banks And S\&Ls \\
\hline 26 & Joyo Bank Limited (The) & Japan & Banks And S\&Ls \\
\hline 27 & Chugoku Bank Limited (The) & Japan & Banks And S\&Ls \\
\hline 28 & Fukuoka Financial Group, Inc & Japan & Banks And S\&Ls \\
\hline 29 & Resona Holdings Inc & Japan & Banks And S\&Ls \\
\hline 30 & Qbe Insurance Group Limited & Australia & Insurance - Prop/Cas/Health \\
\hline
\end{tabular}

Source: Bloomberg LLP and author's calculations. 
Table A2. Emerging markets economies - systemic risk ranking (ranking based on a VAR (8) specification)

\begin{tabular}{clll}
\hline Ranking & Firm & Country & Industry \\
\hline DY rankings & & \\
1 & Sanlam Limited & South Africa & Insurance - Life \\
2 & Banco Do Brasil Sa & Brazil & Banks And S\&Ls \\
3 & Mmi Holdings Limited & South Africa & Insurance - Life \\
4 & Discovery Ltd & South Africa & Insurance - Prop/Cas/Health \\
5 & Firstrand Limited & South Africa & Banks And S\&Ls \\
6 & Standard Bank Group Limited & South Africa & Banks And S\&Ls \\
7 & Banco Bradesco S.A. & Brazil & Banks And S\&Ls \\
8 & Rmb Holdings Limited & South Africa & Insurance - Life \\
9 & Barclays Africa Group Ltd & South Africa & Banks And S\&Ls \\
10 & Porto Seguro Sa & Brazil & Insurance - Prop/Cas/Health \\
11 & Banco Santander Brasil Sa & Brazil & Banks And S\&Ls \\
12 & Nedbank Group Limited & South Africa & Banks And S\&Ls \\
13 & Liberty Holdings Limited & South Africa & Insurance - Prop/Cas/Health \\
14 & Huatai Securities Company Limited & China & Security Brokers \& Dealers \\
15 & Akbank Tas & Turkey & Banks And S\&Ls \\
16 & Turkiye Is Bankasi A.S. & Turkey & Banks And S\&Ls \\
17 & Sul America S.A. & Brazil & Insurance - Prop/Cas/Health \\
18 & Itau Unibanco Holding S.A. & Brazil & Banks And S\&Ls \\
19 & Haci Omer Sabanci Holding A.S. & Turkey & Banks And S\&Ls \\
20 & Turkiye Garanti Bankasi A.S. & Turkey & Banks And S\&Ls \\
21 & Yapi Ve Kredi Bankasi As & Turkey & Banks And S\&Ls \\
22 & Grupo Btg Pactual & Brazil & Security Brokers \& Dealers \\
23 & Santander Mexico Financial Group Sab De Cv & Mexico & Banks And S\&Ls \\
24 & Picc Property And Casualty Company Ltd & China & Insurance - Prop/Cas/Health \\
25 & China Life Insurance Co Ltd & China & Insurance - Life \\
26 & Qualicorp Sa & Brazil & Insurance - Prop/Cas/Health \\
27 & Haitong Securities Co. Limited & China & Security Brokers \& Dealers \\
28 & Ping An Insurance (Group) Company Of China Ltd & China & Insurance - Life \\
29 & Citic Securities Co., Ltd. & China & Security Brokers \& Dealers \\
30 & New China Life Insurance Co Ltd & China & Insurance - Life \\
\hline
\end{tabular}

\begin{tabular}{clll}
\hline Ranking & Firm & Country & Industry \\
\hline CLNDY rankings & & \\
1 & Sanlam Limited & South Africa & Insurance - Life \\
2 & Mmi Holdings Limited & South Africa & Insurance - Life \\
3 & Discovery Ltd & South Africa & Insurance - Prop/Cas/Health \\
4 & Standard Bank Group Limited & South Africa & Banks And S\&Ls \\
5 & Rmb Holdings Limited & South Africa & Insurance - Life \\
6 & Liberty Holdings Limited & South Africa & Insurance - Prop/Cas/Health \\
7 & Nedbank Group Limited & South Africa & Banks And S\&Ls \\
8 & Firstrand Limited & South Africa & Banks And S\&Ls \\
9 & Barclays Africa Group Ltd & South Africa & Banks And S\&Ls \\
10 & Porto Seguro Sa & Brazil & Insurance - Prop/Cas/Health \\
11 & Santander Mexico Financial Group Sab De Cv & Mexico & Banks And S\&Ls \\
12 & China Construction Bank Corp & China & Banks And S\&Ls \\
13 & Picc Property And Casualty Company Ltd & China & Insurance - Prop/Cas/Health \\
14 & Bank Of China Limited & China & Banks And S\&Ls \\
15 & Banco Bradesco S.A. & Brazil & Banks And S\&Ls \\
16 & China Citic Bank Corporation Limited & China & Banks And S\&Ls \\
17 & Bank Of Communications Co Ltd & China & Banks And S\&Ls \\
18 & Ping An Insurance (Group) Company Of China Ltd & China & Insurance - Life \\
19 & Chongqing Rural Commercial Bank Co Ltd & China & Banks And S\&Ls \\
20 & Santam Limited & South Africa & Insurance - Prop/Cas/Health \\
21 & Sul America S.A. & Brazil & Insurance - Prop/Cas/Health \\
22 & Agricultural Bank Of China Limited & China & Banks And S\&Ls \\
23 & Itau Unibanco Holding S.A. & Brazil & Banks And S\&Ls \\
24 & China Life Insurance Co Ltd & China & Insurance - Life \\
25 & The People S Insurance Co (Group) Of Chi & China & Insurance - Prop/Cas/Health \\
26 & Haci Omer Sabanci Holding A.S. & Turkey & Banks And S\&Ls \\
27 & Turkiye Is Bankasi A.S. & Turkey & Banks And S\&Ls \\
28 & New China Life Insurance Co Ltd & China & Insurance - Life \\
29 & Akbank Tas & Turkey & Banks And S\&Ls \\
30 & Yapi Ve Kredi Bankasi As & Turkey & Banks And S\&Ls \\
\hline & & & \\
\end{tabular}

Source: Bloomberg LLP and author's calculations. 
Table A3. Europe - systemic risk ranking

(ranking based on a VAR (8) specification)

\begin{tabular}{|c|c|c|c|c|c|c|c|}
\hline Ranking & Firm & Country & Industry & Ranking & Firm & Country & Industry \\
\hline \multicolumn{4}{|c|}{ DY rankings } & \multicolumn{4}{|c|}{ CLNDY rankings } \\
\hline 1 & Bank Of Piraeus S.A. & Greece & Banks And S\&Ls & 1 & Old Mutual Plc & United Kingdom & Insurance - Life \\
\hline 2 & National Bank Of Greece, S.A. & Greece & Banks And S\&Ls & 2 & Prudential Plc & United Kingdom & Insurance - Life \\
\hline 3 & Eurobank Ergasias Sa & Greece & Banks And S\&Ls & 3 & Societe Generale & France & Banks And S\&Ls \\
\hline 4 & Bankia Sau & Spain & Banks And S\&Ls & 4 & Ubs Group Ag & Switzerland & Banks And S\&Ls \\
\hline 5 & Alpha Bank Sa & Greece & Banks And S\&Ls & 5 & St. James'S Place Plc & United Kingdom & Insurance - Life \\
\hline 6 & Cppgroup Plc & United Kingdom & Insurance - Prop/Cas/Health & 6 & Axa Sa & France & Insurance - Life \\
\hline 7 & Banco Comercial Portugues, S.A. & Portugal & Banks And S\&Ls & 7 & Banco Bilbao Vizcaya Argentaria Sa & Spain & Banks And S\&Ls \\
\hline 8 & Banca Monte Dei Paschi Di Siena Spa & Italy & Banks And S\&Ls & 8 & Hsbc Holdings Plc & United Kingdom & Banks And S\&Ls \\
\hline 9 & Old Mutual Plc & United Kingdom & Insurance - Life & 9 & Banco Santander Sa & Spain & Banks And S\&Ls \\
\hline 10 & Banco Popolare & Italy & Banks And S\&Ls & 10 & Allianz Se & Germany & Insurance - Prop/Cas/Health \\
\hline 11 & Societe Generale & France & Banks And S\&Ls & 11 & Credit Agricole Sa & France & Banks And S\&Ls \\
\hline 12 & Banco Popular Espanol & Spain & Banks And S\&Ls & 12 & Deutsche Bank Aktiengesellschaft & Germany & Banks And S\&Ls \\
\hline 13 & Banco Sabadell & Spain & Banks And S\&Ls & 13 & Aviva Plc & United Kingdom & Insurance - Life \\
\hline 14 & Banca Popolare Dell'Emilia Romagna Sc & Italy & Banks And S\&Ls & 14 & Mapfre Sa & Spain & Insurance - Life \\
\hline 15 & Allied Irish Banks Plc & Ireland & Banks And S\&Ls & 15 & Bnp Paribas Sa & France & Banks And S\&Ls \\
\hline 16 & Ubi Banca & Italy & Banks And S\&Ls & 16 & Ing Groep N.V. & Netherlands & Banks And S\&Ls \\
\hline 17 & Delta Lloyd Nv & Netherlands & Insurance - Life & 17 & European Reliance General Insurance Co. & Greece & Insurance - Life \\
\hline 18 & Credit Agricole Sa & France & Banks And S\&Ls & 18 & Credit Suisse Group Ag & Switzerland & Banks And S\&Ls \\
\hline 19 & St. James'S Place Plc & United Kingdom & Insurance - Life & 19 & Standard Life Plc & United Kingdom & Insurance - Life \\
\hline 20 & Storebrand Asa & Norway & Insurance - Life & 20 & Natixis & France & Banks And S\&Ls \\
\hline 21 & Deutsche Bank Aktiengesellschaft & Germany & Banks And S\&Ls & 21 & Hannover Rueck Se & Germany & Insurance - Life \\
\hline 22 & Unicredit Spa & Italy & Banks And S\&Ls & 22 & Barclays Plc & United Kingdom & Banks And S\&Ls \\
\hline 23 & Banco Santander Sa & Spain & Banks And S\&Ls & 23 & Julius Baer Group Ag & Switzerland & Banks And S\&Ls \\
\hline 24 & Prudential Plc & United Kingdom & Insurance - Life & 24 & Aareal Bank Ag & Germany & Banks And S\&Ls \\
\hline 25 & Natixis & France & Banks And S\&Ls & 25 & Dnb Asa & Norway & Banks And S\&Ls \\
\hline 26 & Standard Chartered Plc & United Kingdom & Banks And S\&Ls & 26 & XI Group Plc -- Adr & Ireland & Insurance - Prop/Cas/Health \\
\hline 27 & Royal Bank Of Scotland Group Plc & United Kingdom & Banks And S\&Ls & 27 & Baloise Holding Ag & Switzerland & Insurance - Life \\
\hline 28 & Mediobanca Spa & Italy & Banks And S\&Ls & 28 & Kbc Group Nv & Belgium & Banks And S\&Ls \\
\hline 29 & Barclays Plc & United Kingdom & Banks And S\&Ls & 29 & Nordea Bank Ab & Sweden & Banks And S\&Ls \\
\hline 30 & Banco Bilbao Vizcaya Argentaria Sa & Spain & Banks And S\&Ls & 30 & Swiss Life Holding Ag & Switzerland & Insurance - Life \\
\hline
\end{tabular}

Source: Bloomberg LLP and author's calculations. 
Table A4. North America - systemic risk ranking (ranking based on a VAR (8) specification)

\begin{tabular}{|c|c|c|c|c|c|c|c|}
\hline Ranking & Firm & Country & Industry & Ranking & Firm & Country & Industry \\
\hline \multicolumn{4}{|c|}{ DY rankings } & \multicolumn{4}{|c|}{ CLNDY rankings } \\
\hline 1 & Genworth Financial Inc & United States & Insurance - Life & 1 & Ameriprise Financial Inc & United States & Security Brokers \& Dealers \\
\hline 2 & Lincoln National Corp & United States & Insurance - Life & 2 & Metlife Inc & United States & Insurance - Life \\
\hline 3 & MBIA Inc & United States & Insurance - Prop/Cas/Health & 3 & Prudential Financial Inc & United States & Insurance - Life \\
\hline 4 & American Eqty Invt Life HIdg & United States & Insurance - Life & 4 & Manulife Financial Corp & Canada & Insurance - Life \\
\hline 5 & UTG Inc & United States & Insurance - Life & 5 & Citigroup Inc & United States & Finance $\mathrm{Nec}$ \\
\hline 6 & Metlife Inc & United States & Insurance - Life & 6 & American International Group & United States & Insurance - Prop/Cas/Health \\
\hline 7 & CNO Financial Group Inc & United States & Insurance - Prop/Cas/Health & 7 & Lincoln National Corp & United States & Insurance - Life \\
\hline 8 & Manulife Financial Corp & Canada & Insurance - Life & 8 & Goldman Sachs Group Inc & United States & Security Brokers \& Dealers \\
\hline 9 & Citigroup Inc & United States & Finance $\mathrm{Nec}$ & 9 & Torchmark Corp & United States & Insurance - Life \\
\hline 10 & Ameriprise Financial Inc & United States & Security Brokers \& Dealers & 10 & Loews Corp & United States & Insurance - Prop/Cas/Health \\
\hline 11 & Prudential Financial Inc & United States & Insurance - Life & 11 & American Financial Group Inc & United States & Insurance - Prop/Cas/Health \\
\hline 12 & Morgan Stanley & United States & Security Brokers \& Dealers & 12 & U S Bancorp & United States & Banks And S\&Ls \\
\hline 13 & State Street Corp & United States & Banks And S\&Ls & 13 & Power Financial Corp & Canada & Insurance - Life \\
\hline 14 & United Insurance Holdings Co & United States & Insurance - Prop/Cas/Health & 14 & Old Republic Intl Corp & United States & Insurance - Prop/Cas/Health \\
\hline 15 & Zions Bancorporation & United States & Banks And S\&Ls & 15 & Sun Life Financial Inc & Canada & Insurance - Life \\
\hline 16 & Mgic Investment Corp/Wi & United States & Insurance - Prop/Cas/Health & 16 & CNO Financial Group Inc & United States & Insurance - Prop/Cas/Health \\
\hline 17 & Industrial Alliance Ins \& Fin & Canada & Insurance - Life & 17 & Suntrust Banks Inc & United States & Banks And S\&Ls \\
\hline 18 & Universal American Corp & United States & Insurance - Prop/Cas/Health & 18 & Humana Inc & United States & Insurance - Prop/Cas/Health \\
\hline 19 & Radian Group Inc & United States & Insurance - Prop/Cas/Health & 19 & Aflac Inc & United States & Insurance - Prop/Cas/Health \\
\hline 20 & Regions Financial Corp & United States & Banks And S\&Ls & 20 & Power Corp Canada & Canada & Insurance - Life \\
\hline 21 & Suntrust Banks Inc & United States & Banks And S\&Ls & 21 & Pnc Financial Svcs Group Inc & United States & Banks And S\&Ls \\
\hline 22 & Bank Of America Corp & United States & Banks And S\&Ls & 22 & JPMorgan Chase \& Co & United States & Banks And S\&Ls \\
\hline 23 & Sun Life Financial Inc & Canada & Insurance - Life & 23 & State Street Corp & United States & Banks And S\&Ls \\
\hline 24 & Comerica Inc & United States & Banks And S\&Ls & 24 & Fifth Third Bancorp & United States & Banks And S\&Ls \\
\hline 25 & $\mathrm{HCl}$ Group Inc & United States & Insurance - Prop/Cas/Health & 25 & Morgan Stanley & United States & Security Brokers \& Dealers \\
\hline 26 & Fifth Third Bancorp & United States & Banks And S\&Ls & 26 & Bank Of New York Mellon Corp & United States & Banks And S\&Ls \\
\hline 27 & Unum Group & United States & Insurance - Prop/Cas/Health & 27 & BB\&T Corp & United States & Banks And S\&Ls \\
\hline 28 & Keycorp & United States & Banks And S\&Ls & 28 & Northern Trust Corp & United States & Banks And S\&Ls \\
\hline 29 & Goldman Sachs Group Inc & United States & Security Brokers \& Dealers & 29 & Bank Of Nova Scotia & Canada & Banks And S\&Ls \\
\hline 30 & National Bank Canada & Canada & Banks And S\&Ls & 30 & Royal Bank Of Canada & Canada & Banks And S\&Ls \\
\hline
\end{tabular}

Source: Bloomberg LLP and author's calculations. 
Table A5. Advanced Asia - systemic vulnerability ranking (ranking based on a VAR (8) specification)

\begin{tabular}{|c|c|c|c|}
\hline Ranking & Firm & Country & Industry \\
\hline \multicolumn{4}{|c|}{ DY rankings } \\
\hline 1 & Taiwan Cooperative Bank & Taiwan & Banks And S\&LS \\
\hline 2 & Taiwan Fire \& Marine Insurance Company L & Taiwan & Insurance - Prop/Cas/Health \\
\hline 3 & Shin Kong Financial Holding Company Limi & Taiwan & Insurance - Life \\
\hline 4 & China Life Insurance Company Limited & Taiwan & Insurance - Life \\
\hline 5 & Shinkong Insurance Co Ltd & Taiwan & Insurance - Prop/Cas/Health \\
\hline 6 & Mercuries Life Insurance Co Ltd & Taiwan & Insurance - Life \\
\hline 7 & Fubon Financial Holding Company Limited & Taiwan & Insurance - Life \\
\hline 8 & First Financial Holding Company Limited & Taiwan & Banks And S\&Ls \\
\hline 9 & First Insurance Company Limited (The) & Taiwan & Insurance - Prop/Cas/Health \\
\hline 10 & Central Reinsurance Corporation & Taiwan & Insurance - Life \\
\hline 11 & Mega Financial Holding Company Limited & Taiwan & Banks And S\&LS \\
\hline 12 & Hua Nan Financial Holdings Company Limit & Taiwan & Banks And S\&Ls \\
\hline 13 & Union Insurance Company Limited & Taiwan & Insurance - Prop/Cas/Health \\
\hline 14 & United Overseas Insurance Limited & Singapore & Insurance - Prop/Cas/Health \\
\hline 15 & Chang Hwa Commercial Bank Limited & Taiwan & Banks And S\&Ls \\
\hline 16 & Cathay Financial Holding Company Limited & Taiwan & Insurance - Life \\
\hline 17 & Lifenet Insurance Company & Japan & Insurance - Life \\
\hline 18 & Great Eastern Holdings Ltd & Singapore & Insurance - Life \\
\hline 19 & Tower Limited & New Zealand & Insurance - Life \\
\hline 20 & Asia Financial Holdings Ltd. & Hong Kong & Insurance - Life \\
\hline 21 & Hang Seng Bank Limited & Hong Kong & Banks And S\&Ls \\
\hline 22 & Min Xin Holdings Ltd. & Hong Kong & Insurance - Prop/Cas/Health \\
\hline 23 & Insurance Australia Group Limited & Australia & Insurance - Prop/Cas/Health \\
\hline 24 & Aia Group Limited & Hong Kong & Insurance - Life \\
\hline 25 & Oversea-Chinese Banking Corporation Limi & Singapore & Banks And S\&Ls \\
\hline 26 & 77 Bank Limited (The) & Japan & Banks And S\&Ls \\
\hline 27 & Dbs Group Holdings Ltd & Singapore & Banks And S\&Ls \\
\hline 28 & Chiba Bank Ltd (The) & Japan & Banks And S\&Ls \\
\hline 29 & Bank Of East Asia Limited (The) & Hong Kong & Banks And S\&Ls \\
\hline 30 & Boc Hong Kong (Holdings) Ltd & Hong Kong & Banks And S\&Ls \\
\hline
\end{tabular}

\begin{tabular}{|c|c|c|c|}
\hline Ranking & Firm & Country & Industry \\
\hline \multicolumn{4}{|c|}{ CLNDY rankings } \\
\hline 1 & Taiwan Cooperative Bank & Taiwan & Banks And S\&Ls \\
\hline 2 & Taiwan Fire \& Marine Insurance Company L & Taiwan & Insurance - Prop/Cas/Health \\
\hline 3 & Shin Kong Financial Holding Company Limi & Taiwan & Insurance - Life \\
\hline 4 & China Life Insurance Company Limited & Taiwan & Insurance - Life \\
\hline 5 & Shinkong Insurance Co Ltd & Taiwan & Insurance - Prop/Cas/Health \\
\hline 6 & Fubon Financial Holding Company Limited & Taiwan & Insurance - Life \\
\hline 7 & Mercuries Life Insurance Co Ltd & Taiwan & Insurance - Life \\
\hline 8 & First Insurance Company Limited (The) & Taiwan & Insurance - Prop/Cas/Health \\
\hline 9 & Union Insurance Company Limited & Taiwan & Insurance - Prop/Cas/Health \\
\hline 10 & Mega Financial Holding Company Limited & Taiwan & Banks And S\&Ls \\
\hline 11 & Lifenet Insurance Company & Japan & Insurance - Life \\
\hline 12 & First Financial Holding Company Limited & Taiwan & Banks And S\&Ls \\
\hline 13 & Central Reinsurance Corporation & Taiwan & Insurance - Life \\
\hline 14 & Hua Nan Financial Holdings Company Limit & Taiwan & Banks And S\&Ls \\
\hline 15 & Cathay Financial Holding Company Limited & Taiwan & Insurance - Life \\
\hline 16 & United Overseas Insurance Limited & Singapore & Insurance - Prop/Cas/Health \\
\hline 17 & Tower Limited & New Zealand & Insurance - Life \\
\hline 18 & Chang Hwa Commercial Bank Limited & Taiwan & Banks And S\&Ls \\
\hline 19 & Great Eastern Holdings Ltd & Singapore & Insurance - Life \\
\hline 20 & Asia Financial Holdings Ltd. & Hong Kong & Insurance - Life \\
\hline 21 & China Taiping Insurance Holdings Company Limited & Hong Kong & Insurance - Life \\
\hline 22 & Min Xin Holdings Ltd. & Hong Kong & Insurance - Prop/Cas/Health \\
\hline 23 & 77 Bank Limited (The) & Japan & Banks And S\&Ls \\
\hline 24 & Chiba Bank Ltd (The) & Japan & Banks And S\&Ls \\
\hline 25 & Nishi-Nippon City Bank Ltd & Japan & Banks And S\&Ls \\
\hline 26 & Insurance Australia Group Limited & Australia & Insurance - Prop/Cas/Health \\
\hline 27 & Fukuoka Financial Group, Inc & Japan & Banks And S\&Ls \\
\hline 28 & Anicom Holdings Incorporation & Japan & Insurance - Prop/Cas/Health \\
\hline 29 & Hiroshima Bank Limited (The) & Japan & Banks And S\&Ls \\
\hline 30 & Shc Insurance Pte Limited & Singapore & Insurance - Prop/Cas/Health \\
\hline
\end{tabular}

Source: Bloomberg LLP and author's calculations. 
Table A6. Emerging markets economies - systemic vulnerability ranking (ranking based on a VAR (8) specification)

\begin{tabular}{|c|c|c|c|}
\hline Ranking & Firm & Country & Industry \\
\hline \multicolumn{4}{|c|}{ DY rankings } \\
\hline 1 & Indequity Group Limited & South Africa & Insurance - Prop/Cas/Health \\
\hline 2 & Jsc Vtb Bank & \multicolumn{2}{|c|}{ Russian Federation Banks And S\&Ls } \\
\hline 3 & Bank Of India & India & Banks And S\&Ls \\
\hline 4 & State Bank Of India & India & Banks And S\&Ls \\
\hline 5 & Axis Bank Limited & India & Banks And S\&Ls \\
\hline 6 & Punjab National Bank Ltd & India & Banks And S\&Ls \\
\hline 7 & Canara Bank & India & Banks And S\&Ls \\
\hline 8 & Hdfc Bank Limited & India & Banks And S\&Ls \\
\hline 9 & Bank Of Baroda & India & Banks And S\&Ls \\
\hline 10 & Aditya Birla Nuvo Limited & India & Insurance - Life \\
\hline 11 & Lippo General Insurance Tbk Pt & Indonesia & Insurance - Prop/Cas/Health \\
\hline 12 & Bajaj Finserv Limited & India & Insurance - Life \\
\hline 13 & Icici Bank Limited & India & Banks And S\&Ls \\
\hline 14 & Grupo Financiero Banorte S.A.B. De C.V. & Mexico & Banks And S\&Ls \\
\hline 15 & Industrial Bank Co Ltd & China & Banks And S\&Ls \\
\hline 16 & Asuransi Harta Aman Pratama Tbk Pt & Indonesia & Insurance - Prop/Cas/Health \\
\hline 17 & Sberbank Rossii Oao & \multicolumn{2}{|c|}{ Russian Federation Banks And S\&LS } \\
\hline 18 & Aviva Sigorta As & Turkey & Insurance - Prop/Cas/Health \\
\hline 19 & Anadolu Anonim Turk Sigorta Sirketi & Turkey & Insurance - Prop/Cas/Health \\
\hline 20 & Bank Rakyat Indonesia (Persero) Tbk Pt & Indonesia & Banks And S\&Ls \\
\hline 21 & Bank Mandiri (Persero) Tbk Pt & Indonesia & Banks And S\&Ls \\
\hline 22 & Picc Property And Casualty Company Ltd & China & Insurance - Prop/Cas/Health \\
\hline 23 & Paninvest Tbk Pt & Indonesia & Insurance - Life \\
\hline 24 & Anadolu Hayat Emeklilik A.S. & Turkey & Insurance - Life \\
\hline 25 & Turkiye Garanti Bankasi A.S. & Turkey & Banks And S\&Ls \\
\hline 26 & Aksigorta Anonim Sirketi & Turkey & Insurance - Prop/Cas/Health \\
\hline 27 & New China Life Insurance Co Ltd & China & Insurance - Life \\
\hline 28 & Yapi Ve Kredi Bankasi As & Turkey & Banks And S\&Ls \\
\hline 29 & Bank Central Asia Tbk Pt & Indonesia & Banks And S\&Ls \\
\hline 30 & Akbank Tas & Turkey & Banks And S\&Ls \\
\hline
\end{tabular}

\begin{tabular}{clll}
\hline Ranking & Firm & Country & Industry \\
\hline CLNDY rankings & & \\
1 & Indequity Group Limited & South Africa & Insurance - Prop/Cas/Health \\
2 & State Bank Of India & India & Banks And S\&Ls \\
3 & Jsc Vtb Bank & Russian Federation Banks And S\&Ls \\
4 & Bank Of India & India & Banks And S\&Ls \\
5 & Axis Bank Limited & India & Banks And S\&Ls \\
6 & Hdfc Bank Limited & India & Banks And S\&Ls \\
7 & Punjab National Bank Ltd & India & Banks And S\&Ls \\
8 & Canara Bank & India & Banks And S\&Ls \\
9 & Aditya Birla Nuvo Limited & India & Insurance - Life \\
10 & Bajaj Finserv Limited & India & Insurance - Life \\
11 & Bank Of Baroda & India & Banks And S\&Ls \\
12 & Grupo Financiero Banorte S.A.B. De C.V. & Mexico & Banks And S\&Ls \\
13 & Icici Bank Limited & India & Banks And S\&Ls \\
14 & Lippo General Insurance Tbk Pt & Indonesia & Insurance - Prop/Cas/Health \\
15 & Anadolu Anonim Turk Sigorta Sirketi & Turkey & Insurance - Prop/Cas/Health \\
16 & Industrial Bank Co Ltd & China & Banks And S\&Ls \\
17 & Paninvest Tbk Pt & Indonesia & Insurance - Life \\
18 & Sberbank Rossii Oao & Russian Federation Banks And S\&Ls \\
19 & Picc Property And Casualty Company Ltd & China & Insurance - Prop/Cas/Health \\
20 & Aviva Sigorta As & Turkey & Insurance - Prop/Cas/Health \\
21 & Investec Limited & South Africa & Security Brokers \& Dealers \\
22 & Anadolu Hayat Emekilik A.S. & Turkey & Insurance - Life \\
23 & Bank Mandiri (Persero) Tbk Pt & Indonesia & Banks And S\&Ls \\
24 & Bank Central Asia Tbk Pt & Indonesia & Banks And S\&Ls \\
25 & Agricultural Bank Of China Limited & China & Banks And S\&Ls \\
26 & Aksigorta Anonim Sirketi & Turkey & Insurance - Prop/Cas/Health \\
27 & Bank Rakyat Indonesia (Persero) Tbk Pt & Indonesia & Banks And S\&Ls \\
28 & New China Life Insurance Co Ltd & China & Insurance - Life \\
29 & Ray Sigorta A.S. & Turkey & Insurance - Prop/Cas/Health \\
30 & China Construction Bank Corp & China & Banks And S\&Ls \\
\hline & & & \\
& &
\end{tabular}

Source: Bloomberg LLP and author's calculations. 
Table A7. Europe - systemic vulnerability ranking (ranking based on a VAR (8) specification)

\begin{tabular}{|c|c|c|c|}
\hline Ranking & Firm & Country & Industry \\
\hline \multicolumn{4}{|c|}{ DY rankings } \\
\hline 1 & CPPgroup PIc & United Kingdom & Insurance - Prop/Cas/Health \\
\hline 2 & FBD Holdings Plc & Ireland & Insurance - Prop/Cas/Health \\
\hline 3 & Nuernberger Beteiligungs-Ag & Germany & Insurance - Life \\
\hline 4 & Wuestenrot \& Wuerttembergische Aktienges & Germany & Insurance - Life \\
\hline 5 & Credit Industriel Et Commercial Sa & France & Banks And S\&Ls \\
\hline 6 & Randall And Quilter Investment Holdings & United Kingdom & Insurance - Prop/Cas/Health \\
\hline 7 & Helios Underwriting Plc & United Kingdom & Insurance - Life \\
\hline 8 & Deutsche Postbank Ag & Germany & Banks And S\&Ls \\
\hline 9 & Personal Group Holdings Plc & United Kingdom & Insurance - Prop/Cas/Health \\
\hline 10 & Amlin Plc & United Kingdom & Insurance - Prop/Cas/Health \\
\hline 11 & Admiral Group Plc & United Kingdom & Insurance - Prop/Cas/Health \\
\hline 12 & Novae Group Plc & United Kingdom & Insurance - Prop/Cas/Health \\
\hline 13 & Phoenix Group Holdings & Netherlands & Insurance - Life \\
\hline 14 & Allied World Assurance Co Ag-Adr & Switzerland & Insurance - Prop/Cas/Health \\
\hline 15 & Euler Hermes Group Sa & France & Insurance - Prop/Cas/Health \\
\hline 16 & Ackermans \& Van Haaren & Belgium & Insurance - Prop/Cas/Health \\
\hline 17 & Global Indemnity Plc-Adr & Ireland & Insurance - Prop/Cas/Health \\
\hline 18 & Hiscox Plc & United Kingdom & Insurance - Prop/Cas/Health \\
\hline 19 & Hansard Global Plc & United Kingdom & Insurance - Life \\
\hline 20 & Swiss Re Ag & Switzerland & Insurance - Life \\
\hline 21 & Interlife General Insurance Company Sa & Greece & Insurance - Prop/Cas/Health \\
\hline 22 & Vittoria Assicurazioni Spa & Italy & Insurance - Prop/Cas/Health \\
\hline 23 & Beazley Plc & United Kingdom & Insurance - Prop/Cas/Health \\
\hline 24 & Helvetia Holding Ag & Switzerland & Insurance - Life \\
\hline 25 & Chesnara Plc & United Kingdom & Insurance - Prop/Cas/Health \\
\hline 26 & Uniqa Insurance Group Ag & Austria & Insurance - Life \\
\hline 27 & Zurich Insurance Group Limited & Switzerland & Insurance - Prop/Cas/Health \\
\hline 28 & Direct Line Insurance Group Plc & United Kingdom & Insurance - Prop/Cas/Health \\
\hline 29 & Baloise Holding Ag & Switzerland & Insurance - Life \\
\hline 30 & Danske Bank As & Denmark & Banks And S\&Ls \\
\hline
\end{tabular}

\begin{tabular}{|c|c|c|c|}
\hline Ranking & Firm & Country & Industry \\
\hline \multicolumn{4}{|c|}{ CLNDY rankings } \\
\hline 1 & CPPgroup Plc & United Kingdom & Insurance - Prop/Cas/Health \\
\hline 2 & FBD Holdings Plc & Ireland & Insurance - Prop/Cas/Health \\
\hline 3 & Randall And Quilter Investment Holdings & United Kingdom & Insurance - Prop/Cas/Health \\
\hline 4 & Wuestenrot \& Wuerttembergische Aktienges & Germany & Insurance - Life \\
\hline 5 & Interlife General Insurance Company Sa & Greece & Insurance - Prop/Cas/Health \\
\hline 6 & Helios Underwriting Plc & United Kingdom & Insurance - Life \\
\hline 7 & Allied Irish Banks Plc & Ireland & Banks And S\&Ls \\
\hline 8 & Nuernberger Beteiligungs-Ag & Germany & Insurance - Life \\
\hline 9 & Credit Industriel Et Commercial Sa & France & Banks And S\&Ls \\
\hline 10 & Eurobank Ergasias Sa & Greece & Banks And S\&Ls \\
\hline 11 & Deutsche Postbank Ag & Germany & Banks And S\&Ls \\
\hline 12 & Admiral Group Plc & United Kingdom & Insurance - Prop/Cas/Health \\
\hline 13 & Amlin Plc & United Kingdom & Insurance - Prop/Cas/Health \\
\hline 14 & Personal Group Holdings Plc & United Kingdom & Insurance - Prop/Cas/Health \\
\hline 15 & Hansard Global Plc & United Kingdom & Insurance - Life \\
\hline 16 & Novae Group Plc & United Kingdom & Insurance - Prop/Cas/Health \\
\hline 17 & Phoenix Group Holdings & Netherlands & Insurance - Life \\
\hline 18 & Unicredit Spa & Italy & Banks And S\&LS \\
\hline 19 & Alpha Bank Sa & Greece & Banks And S\&Ls \\
\hline 20 & Bank Of Ireland & Ireland & Banks And S\&Ls \\
\hline 21 & Unipolsai Assicurazioni Spa & Italy & Insurance - Prop/Cas/Health \\
\hline 22 & Delta Lloyd Nv & Netherlands & Insurance - Life \\
\hline 23 & Permanent Tsb Group Holdings Plc & Ireland & Insurance - Life \\
\hline 24 & Intesa Sanpaolo Spa & Italy & Banks And S\&Ls \\
\hline 25 & Beazley Plc & United Kingdom & Insurance - Prop/Cas/Health \\
\hline 26 & Euler Hermes Group Sa & France & Insurance - Prop/Cas/Health \\
\hline 27 & Chesnara Plc & United Kingdom & Insurance - Prop/Cas/Health \\
\hline 28 & Uniqa Insurance Group Ag & Austria & Insurance - Life \\
\hline 29 & Hiscox Plc & United Kingdom & Insurance - Prop/Cas/Health \\
\hline 30 & Lloyds Banking Group Plc & United Kingdom & Banks And S\&Ls \\
\hline
\end{tabular}

Source: Bloomberg LLP and author's calculations. 
Table A8. North America - systemic vulnerability ranking (ranking based on a VAR (8) specification)

\begin{tabular}{|c|c|c|c|}
\hline Ranking & Firm & Country & Industry \\
\hline \multicolumn{4}{|c|}{ DY rankings } \\
\hline 1 & Independence Holding Co & United States & Insurance - Prop/Cas/Health \\
\hline 2 & White Mtns Ins Group Ltd & Bermuda & Insurance - Prop/Cas/Health \\
\hline 3 & Kingstone $\operatorname{Cos} \operatorname{Inc}$ & United States & Insurance - Prop/Cas/Health \\
\hline 4 & Partnerre Ltd & Bermuda & Insurance - Prop/Cas/Health \\
\hline 5 & Federated National HIdg Co & United States & Insurance - Prop/Cas/Health \\
\hline 6 & Axis Capital Holdings Ltd & Bermuda & Insurance - Prop/Cas/Health \\
\hline 7 & Donegal Group Inc & United States & Insurance - Prop/Cas/Health \\
\hline 8 & Kansas City Life Ins Co & United States & Insurance - Life \\
\hline 9 & First Acceptance Corp & United States & Insurance - Prop/Cas/Health \\
\hline 10 & Proassurance Corp & United States & Insurance - Prop/Cas/Health \\
\hline 11 & Markel Corp & United States & Insurance - Prop/Cas/Health \\
\hline 12 & Stancorp Financial Group Inc & United States & Insurance - Prop/Cas/Health \\
\hline 13 & Fairfax Financial Holdings & Canada & Insurance - Prop/Cas/Health \\
\hline 14 & Arch Capital Group Ltd & Bermuda & Insurance - Prop/Cas/Health \\
\hline 15 & Phoenix Companies Inc & United States & Insurance - Life \\
\hline 16 & Anthem Inc & United States & Insurance - Prop/Cas/Health \\
\hline 17 & Kingsway Financial Svcs Inc & Canada & Insurance - Prop/Cas/Health \\
\hline 18 & Renaissancere Holdings Ltd & Bermuda & Insurance - Prop/Cas/Health \\
\hline 19 & Cigna Corp & United States & Insurance - Prop/Cas/Health \\
\hline 20 & Baldwin \& Lyons $-\mathrm{Cl} \mathrm{B}$ & United States & Insurance - Prop/Cas/Health \\
\hline 21 & Onebeacon Insurance Group & Bermuda & Insurance - Prop/Cas/Health \\
\hline 22 & Infinity Property \& Cas Corp & United States & Insurance - Prop/Cas/Health \\
\hline 23 & Interactive Brokers Group & United States & Security Brokers \& Dealers \\
\hline 24 & National Western Life -Cl A & United States & Insurance - Life \\
\hline 25 & Erie Indemnity $\mathrm{Co}-\mathrm{Cl} \mathrm{A}$ & United States & Insurance - Prop/Cas/Health \\
\hline 26 & Hallmark Financial Services & United States & Insurance - Prop/Cas/Health \\
\hline 27 & Echelon Financial HIdgs Inc & Canada & Insurance - Prop/Cas/Health \\
\hline 28 & Emc Insurance Group Inc & United States & Insurance - Prop/Cas/Health \\
\hline 29 & Endurance Specialty Holdings & Bermuda & Insurance - Prop/Cas/Health \\
\hline 30 & Everest Re Group Ltd & Bermuda & Insurance - Prop/Cas/Health \\
\hline
\end{tabular}

\begin{tabular}{|c|c|c|c|}
\hline Ranking & Firm & Country & Industry \\
\hline \multicolumn{4}{|c|}{ CLNDY rankings } \\
\hline 1 & Phoenix Companies Inc & United States & Insurance - Life \\
\hline 2 & Independence Holding Co & United States & Insurance - Prop/Cas/Health \\
\hline 3 & Federated National HIdg Co & United States & Insurance - Prop/Cas/Health \\
\hline 4 & First Acceptance Corp & United States & Insurance - Prop/Cas/Health \\
\hline 5 & Kingstone Cos Inc & United States & Insurance - Prop/Cas/Health \\
\hline 6 & White Mtns Ins Group Ltd & Bermuda & Insurance - Prop/Cas/Health \\
\hline 7 & Kingsway Financial Svcs Inc & Canada & Insurance - Prop/Cas/Health \\
\hline 8 & Kansas City Life Ins Co & United States & Insurance - Life \\
\hline 9 & Stancorp Financial Group Inc & United States & Insurance - Prop/Cas/Health \\
\hline 10 & Donegal Group Inc & United States & Insurance - Prop/Cas/Health \\
\hline 11 & Hallmark Financial Services & United States & Insurance - Prop/Cas/Health \\
\hline 12 & Cigna Corp & United States & Insurance - Prop/Cas/Health \\
\hline 13 & National Sec Group Inc & United States & Insurance - Prop/Cas/Health \\
\hline 14 & National Interstate Corp & United States & Insurance - Prop/Cas/Health \\
\hline 15 & Mbia Inc & United States & Insurance - Prop/Cas/Health \\
\hline 16 & Anthem Inc & United States & Insurance - Prop/Cas/Health \\
\hline 17 & Partnerre Ltd & Bermuda & Insurance - Prop/Cas/Health \\
\hline 18 & Interactive Brokers Group & United States & Security Brokers \& Dealers \\
\hline 19 & Fairfax Financial Holdings & Canada & Insurance - Prop/Cas/Health \\
\hline 20 & Axis Capital Holdings Ltd & Bermuda & Insurance - Prop/Cas/Health \\
\hline 21 & Emc Insurance Group Inc & United States & Insurance - Prop/Cas/Health \\
\hline 22 & United Insurance Holdings Co & United States & Insurance - Prop/Cas/Health \\
\hline 23 & Utg Inc & United States & Insurance - Life \\
\hline 24 & Triple-S Management Corp & United States & Insurance - Prop/Cas/Health \\
\hline 25 & National Western Life -Cl A & United States & Insurance - Life \\
\hline 26 & Universal American Corp & United States & Insurance - Prop/Cas/Health \\
\hline 27 & Echelon Financial HIdgs Inc & Canada & Insurance - Prop/Cas/Health \\
\hline 28 & Proassurance Corp & United States & Insurance - Prop/Cas/Health \\
\hline 29 & Wmih Corp & United States & Insurance - Prop/Cas/Health \\
\hline 30 & Wellcare Health Plans Inc & United States & Insurance - Prop/Cas/Health \\
\hline
\end{tabular}

Source: Bloomberg LLP and author's calculations. 